\title{
Reactive metal-oxide interfaces: A microscopic view
}

\author{
A. Picone*, M. Riva, A. Brambilla, A. Calloni, G. Bussetti, M. Finazzi, F. Ciccacci, L. Duō** \\ Dipartimento di Fisica, Politecnico di Milano, piazza L. da Vinci 32, 20133 Milano, Italy
}

\begin{abstract}
Metal-oxide interfaces play a fundamental role in determining the functional properties of artificial layered heterostructures, which are at the root of present and future technological applications. Magnetic exchange and magnetoelectric coupling, spin filtering, metal passivation, catalytic activity of oxide-supported nano-particles are just few examples of physical and chemical processes arising at metal-oxide hybrid systems, readily exploited in working devices. These phenomena are strictly correlated with the chemical and structural characteristics of the metal-oxide interfacial region, making a thorough understanding of the atomistic mechanisms responsible of its formation a prerequisite in order to tailor the device properties. The steep compositional gradient established upon formation of metal-oxide heterostructures drives strong chemical interactions at the interface, making the metal-oxide boundary region a complex system to treat, both from an experimental and a theoretical point of view. However, once properly mastered, interfacial chemical interactions offer a further degree of freedom for tuning the material properties. The goal of the present review is to provide a summary of the latest achievements in the understanding of metal/oxide and oxide/metal layered systems characterized by reactive interfaces. The influence of the interface composition on the structural, electronic and magnetic properties will be highlighted. Particular emphasis will be devoted to the discussion of ultra-thin epitaxial oxides stabilized on highly oxidizable metals, which have been rarely exploited as oxide supports as compared to the much more widespread noble and quasi noble metallic substrates. In this frame, an extensive discussion is devoted to the microscopic characterization of interfaces between epitaxial metal oxides and the Fe( 001$)$ substrate, regarded from the one hand as a prototypical ferromagnetic material and from the other hand as a highly oxidizable metal.
\end{abstract}

(c) 2016 Elsevier B.V. All rights reserved.

Keywords: Scanning tunneling microscopy and spectroscopy; X-ray photoemission spectroscopy; Molecular beam epitaxy; Metal-oxide interfaces; Oxide surfaces

\section{Nomenclature}

DFT density functional theory

$E_{\mathrm{F}} \quad$ Fermi energy

L Langmuir

LEED low energy electron diffraction

MBE molecular beam epitaxy
ML monolayer

STM scanning tunneling microscopy

STS scanning tunneling spectroscopy

UHV ultra-high vacuum

UPS ultraviolet photoemission spectroscopy

XMCD X-ray magnetic circular dichroism

XPS $\quad \mathrm{x}$-ray photoemission spectroscopy

\footnotetext{
*Corresponding author. Tel. : +3902 23996089.

**Corresponding author. Tel.: +3902 23996179 .

E-mail addresses: andrea.picone@polimi.it (A. Picone), lamberto.duo@polimi.it (L. Duò).
} 


\section{Introduction}

Interface formation between metals and oxides are one of the most widely investigated topic in physics, chemistry and material science. The reason for this huge interest is that there is almost no technological field in which the metal-oxide interaction does not play a prominent role, including (nano) catalysis [1-3], microelectronics [4,5], magnetic storage media [6], and protective coatings against corrosion $[7,8]$.

From a more fundamental point of view, the metal-oxide interface represents an interesting example of boundary between two materials possessing markedly different and often antithetical electronic and structural properties [9]. As a relevant case, let us mention that a few nanometer-thick (hydro)oxide skin forms at the surface of almost every metal exposed to environmental conditions [10]. The characteristics of this thin surface oxide layer and its interaction with the underlying metallic support are often key factors in determining the physical and chemical properties of metal surfaces.

The material science community has also dedicated a remarkable attention to the investigation, both theoretical and experimental, of heteroepitaxial metal-oxide systems, where, at variance from native oxides, the metal element is different from the cationic species of the oxide phase. Many physical phenomena arising in artificial metal-oxide heterostructures have been reported, spanning from magnetic instabilities [11,12], charge transfer across the metal-oxide interface [13], magnetoelectric coupling in layered multiferroic structures [14], and tunneling-induced spin filtering [15,16], just to mention a few. All these phenomena are intimately connected with the structural and chemical characteristics of the metal-oxide interfacial region. In order to control, and to some extent tuning, the functionalities of these heterostructures, it is of crucial importance to gain an in-depth understanding of the mechanisms driving the formation of metal-oxides interfaces, in terms of both thermodynamical stability and kinetic constraints imposed by the specific preparation method.

Two different classes of systems can be identified, the metal/oxide $(\mathrm{M} / \mathrm{O})$ and the oxide/metal $(\mathrm{O} / \mathrm{M})$ heterophases, sketched in Fig. 1. These two seemingly symmetric interfaces often possess different structural and chemical characteristics, mainly because different are the experimental procedures exploited for their preparation (see Section 2.1). The $\mathrm{O} / \mathrm{M}$ interface is grown by depositing ultra-thin (0$10 \mathrm{~nm}$ thick) oxide films on metallic substrates, exploiting the so-called reactive deposition, in which metal atoms are deposited in the presence of an oxidizing atmosphere [17]. On the other hand, the M/O interface can be obtained by depositing metallic films on well-defined oxide surfaces $[18,19]$. In the latter case, in order to alleviate charging problems related to the use of electron-based microscopic and spectroscopic techniques, the oxide layer of the $\mathrm{M} / \mathrm{O}$ structure is often supported by a metallic substrate. Despite beyond a critical thickness the surface properties of these metal-supported ultra-thin oxide films have proven to mimic those of the corresponding bulk compounds [20,21], for few unit-cell-thick oxide films the influence of the bottom metallic substrate on the M/O properties cannot be neglected, so that the system is better described as a M/O/ $M$ layered structure.

Besides the different experimental constraints characterizing their preparation, the $\mathrm{O} / \mathrm{M}$ and $\mathrm{M} / \mathrm{O}$ interfaces bear also intrinsic differences, since the surface free energy of oxidic compounds is generally lower with respect to that of metallic elements (see Section 2.1). For this reason, oxide films tend to wet metallic substrates, while metals deposited on oxide surfaces are prone to clustering, or even to being encapsulated by a thin oxide layer. Such a fundamentally different growth mode observed in the early stages of formation of $\mathrm{M} / \mathrm{O}$ and $\mathrm{O} / \mathrm{M}$ interfaces can remarkably influence their final morpho-logical and chemical characteristics.

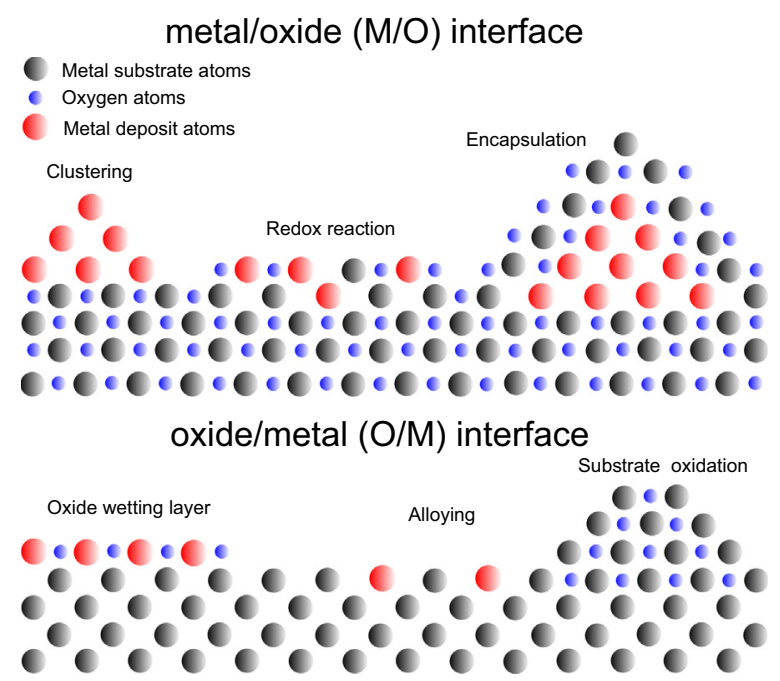

Fig. 1. Metal/oxide (M/O) and oxide/metal (O/M) interfaces. The main phenomena occurring in the early stages of interface formation are displayed. 
In this work, we will provide a survey of the mechanisms governing the formation of $\mathrm{O} / \mathrm{M}$ and $\mathrm{M} / \mathrm{O}$ interfaces in those cases in which these interfaces cannot be considered chemically and structurally sharp. Of course every real interface is characterized by a certain degree of defectiveness and intermixing, so that a perfectly abrupt metal-oxide interface is not realizable in practice. However, in the present review the focus will be on those cases where the chemical and structural inhomogeneities at the metal-oxide interface are the prominent driving forces determining the final heteroepitaxial system structure and its physico-chemical properties. Such chemical and structural inhomogeneities are often regarded as an unwelcome side effects occurring during the preparation of the heterostructures, because the presence of complex phases at the $\mathrm{M} / \mathrm{O}(\mathrm{O} / \mathrm{M})$ boundary makes the interpretation of the experimental data and the theoretical modeling a much more difficult task than for ideally abrupt interfaces. Nevertheless, once properly mastered, such "imperfect interfaces" could represent an opportunity to tune the chemical and physical properties of the nano-structures under consideration.

Particular emphasis will be devoted to the discussion of $\mathrm{O} / \mathrm{M}$ systems in which the metallic substrate possesses a low electronegativity, i.e. a high oxygen affinity. To this class of materials belong in particular the magnetic $3 d$ transition metals such as $\mathrm{Fe}, \mathrm{Co}$ and $\mathrm{Ni}$, which are largely exploited in spintronic devices and for magnetic storage applications. Due to their high reactivity, little is known about the atomic scale mechanisms driving the formation of epitaxial oxide on these substrates, as compared to the large body of literature focusing on the preparation and characterization of ultra-thin oxide films on inert materials such as noble and quasi noble metals.

The review is organized as follows. In Section 2 the main physical processes driven by the chemical reactions occurring at the $\mathrm{M} / \mathrm{O}$ and $\mathrm{O} / \mathrm{M}$ interface are introduced. Section 3 describes the growth and characterization of ultra-thin oxides stabilized on highly oxidizable substrates. Section 4 focuses on transition metal oxides grown on $\mathrm{Fe}(001)$, a topic which has been thoroughly investigated by the authors. Conclusions and perspectives are given in Section 5.

\section{Physics and chemistry at reactive metal-oxide interfaces}

\subsection{Interface chemical reactions}

The main chemical interaction between a metal and an oxide upon interface formation is the redox reaction, in which few atomic planes of the metallic layer in contact with the oxide surface become oxidized, while the oxide cations reduce their oxidation state. The process is driven by charge transfer and migration of cationic and/or anionic species across the metaloxide interface.

Starting from a metallic and an oxide layer, the metal oxidation and the oxide reduction upon interface formation are described by the reaction $\mathrm{M}^{\mathrm{a}}+\mathrm{M}^{\mathrm{b}} \mathrm{O}_{y} \rightarrow \mathrm{M}^{\mathrm{a}} \mathrm{O}_{x}+\mathrm{M}^{\mathrm{b}} \mathrm{O}_{y-x}$. As already pointed out by Campbell [18] and Fu et al. [19] for the $\mathrm{M} / \mathrm{O}$ interface, the most appropriate parameter to understand whether a redox reaction can occur upon interface formation is the oxide standard heat of formation per mole of oxygen $\left(\Delta H_{\mathrm{f}}^{0}\right)$, which has to be considered for the most stable oxide of the elements involved in the interface formation. $\Delta H_{\mathrm{f}}^{0}$ corresponds to the energy gain for the formation of an oxide compound starting from molecular oxygen and metal atoms, with all substances in their standard states, at $T=298.15 \mathrm{~K}$ and $p=100 \mathrm{kPa}$. The lower $\Delta H_{\mathrm{f}}^{0}$, the higher the energy released during the formation of the corresponding oxide. Table 1 reports the $\Delta H_{\mathrm{f}}^{0}$ values for some oxide compounds that will be discussed in the present review.

Despite the $\Delta H_{\mathrm{f}}^{0}$ values provide a useful guide to predict if the reaction is thermodynamically favored and can be expected to occur when the corresponding interface is formed, additional considerations should be taken into account. First of all, even in cases where there is no transfer of oxygen atoms from the oxide to the metallic lattice, the metal proximity can influence the stoichiometric composition of the oxide interfacial layer, as a result of charge transfer from the metal to the oxide layer (or vice-versa). This effect should be taken into account especially for interfaces between oxides and inert metals, such as noble metals, for which the small $\Delta H_{\mathrm{f}}^{0}$ absolute values of the corresponding oxide compounds would suggest a negligible effect on the oxide composition. Charge transfer is expected to be more significant on reducible oxides, such as transition metal or rare earth oxides. On the other hand, for wide gap insulators such as $\mathrm{MgO}, \mathrm{CaO}$, and $\mathrm{Al}_{2} \mathrm{O}_{3}$, characterized by a high ionization potential and a low electron affinity, the effect is limited [24].

A noteworthy phenomenon that might be observed in association with charge transfer across an $\mathrm{O} / \mathrm{M}$ interface is the formation of reduced species in the interfacial oxide region. This might happen, for instance, when $\mathrm{Au}$, for which $\Delta H_{\mathrm{f}}^{0}$ is positive (i.e. the oxidic compound is not stable [18]), is employed as a substrate for the stabilization of ultra-thin oxides. As an example we might mention the $\mathrm{MoO}_{3} / \mathrm{Au}$ interface, where Mo cations are reduced from a $6+$ to a formal $5+$ oxidation state [25]. Another case is represented by 
Table 1

Standard enthalpy (heat) of formation $\Delta H_{\mathrm{f}}^{0}$ per mole of oxygen at $T=298.15 \mathrm{~K}$ and $p=100 \mathrm{kPa}$. The more negative $\Delta H_{\mathrm{f}}^{0}$, the more energetically favored the oxide formation. Adapted from [22].

\begin{tabular}{ll}
\hline Oxide & $\Delta H_{\mathrm{f}}^{0}[\mathrm{~kJ} /(\mathrm{molO})]$ \\
\hline $\mathrm{Co}_{3} \mathrm{O}_{4}$ & -222.75 \\
$\mathrm{CoO}$ & -237.9 \\
$\mathrm{NiO}$ & $-240.6[23]$ \\
$\mathrm{FeO}$ & -272 \\
$\mathrm{Fe}_{2} \mathrm{O}_{3}$ & -274.7 \\
$\mathrm{Fe}_{3} \mathrm{O}_{4}$ & -279.6 \\
$\mathrm{MoO}_{2}$ & -294.4 \\
$\mathrm{WO}_{2}$ & -294.8 \\
$\mathrm{Cr}_{2} \mathrm{O}_{3}$ & -379.9 \\
$\mathrm{Cr}_{3} \mathrm{O}_{4}$ & -382.75 \\
$\mathrm{MnO}$ & -385.2 \\
$\mathrm{BaO}_{\mathrm{Al}} \mathrm{O}_{3}$ & -548 \\
$\mathrm{MgO}$ & -558.57 \\
$\mathrm{CaO}$ & -601.6 \\
\hline
\end{tabular}

$\mathrm{TiO}_{2}$ ultra-thin films grown on $\mathrm{Au}(100)$, which are characterized by the presence of reduced oxide species $\left(\mathrm{Ti}^{3+}\right.$ ions $)$ at the interface, as deduced from the evolution of the lineshape of the Ti $2 p$ XPS peaks as a function of the oxide coverage [26]. The $\mathrm{WO}_{3}$ wetting layer covering a $\mathrm{Pt}(111)$ substrate is also characterized by the presence of tungsten atoms with a reduced $(5+)$ oxidation state [27].

Similarly, for the reverse case of M/O interfaces, De Masi et al. [28] and Benedetti et al. [29] found that Au deposited on the $\mathrm{NiO}(001)$ surface induces the reduction of a small fraction of surface $\mathrm{Ni}$ atoms from the oxidation state +2 to 0 . Analogous results were obtained for $\mathrm{Ag}$ nanoparticles deposited on stoichiometric $\mathrm{CeO}_{2}(111)$, where the Ag-induced reduction of $\mathrm{Ce}$ cations from the oxidation state +4 to +3 has been detected [30].

Charge transfer can also promote the stabilization of oxide layers where the cation is characterized by a higher oxidation state than the one it typically assumes in stable bulk oxides. For instance, vanadium oxide thin films possessing a formal $\mathrm{VO}_{3}$ stoichiometry have been grown on $\mathrm{Rh}(111)$ [31,32]. Since the maximum oxidation state of vanadium is $5+$, electron transfer from the metallic substrate to the oxide overlayer has been invoked to explain such a high oxidation state. Similarly, charge transfer at the O/M interface has been proposed to explain the formation of highly oxidized Co oxide islands on $\mathrm{Au}(111)$ [33]. More specifically, investigating the influence of oxygen pressure on the structural and chemical composition of $\mathrm{Au}(111)$-supported Co oxide islands, Walton et al. found that $\mathrm{CoO}$ islands are mainly stabilized at low oxygen pressure, with Co cations in a $2+$ oxidation state. When Co deposition is performed at higher oxygen pressures, an extra layer of oxygen atoms is added at the interface with the $\mathrm{Au}$ (111) substrate, resulting in a trilayer $\mathrm{O}-\mathrm{Co}-\mathrm{O}$ structure, with Co cations in a formal $3+$ oxidation state [33].

Beside charge transfer, a second issue to be considered to understand $\mathrm{M} / \mathrm{O}$ and $\mathrm{O} / \mathrm{M}$ interface formation is that these apparently identical interfaces often do not display the same chemical composition. As we mentioned in the Introduction, this stems from the different preparation protocols. Generally, metal-supported ultra-thin oxide films are grown by reactive deposition, i.e. by means of MBE performed in an oxidizing atmosphere, while $\mathrm{M} / \mathrm{O}$ heterostructures are obtained by direct metal evaporation onto well-defined oxide substrates in UHV conditions. The main difference between these two procedures consists in the different oxygen availability during the growth, as well as its physical and chemical states (gas in the case of reactive deposition, bound to cations in the case of metals deposited on oxide surfaces).

A paradigmatic example of such an asymmetry can be recognized in the case of $\mathrm{NiO} / \mathrm{Fe}(\mathrm{Fe} / \mathrm{NiO})$ interfaces. The interfacial structure of ultra-thin $\mathrm{Fe}$ films deposited on $\mathrm{NiO}$ has been deeply investigated by means of spectroscopic and diffraction techniques [34-39], as well as modeled by ab initio DFT calculations $[37,40]$. These studies reveal that, upon Fe deposition on the $\mathrm{NiO}(001)$ surface, an interfacial $\mathrm{FeO}$ layer is formed and $\mathrm{NiO}$ is reduced to metallic $\mathrm{Ni}$, with the formation of an alloyed $\mathrm{NiFe}$ layer [37].

On the other hand, the reverse $\mathrm{NiO} / \mathrm{Fe}$ interface, obtained by means of reactive deposition of $\mathrm{Ni}$ on the $\mathrm{Fe}(001)$ surface, is characterized by the development of a thick $\mathrm{Fe}_{3} \mathrm{O}_{4}$ oxide [4143], while no metallic $\mathrm{Ni}$ is detected at the interface [42]. Upon annealing, $\mathrm{Fe}_{3} \mathrm{O}_{4}$ reduction to $\mathrm{FeO}$ is observed, along with the reduction of $\mathrm{NiO}$ and dissolution of $\mathrm{Ni}$ atoms into the $\mathrm{Fe}(001)$ bulk $[42,41]$. The different chemical compositions of $\mathrm{Fe} / \mathrm{NiO}$ and $\mathrm{NiO} / \mathrm{Fe}$ interfaces can be easily rationalized considering that, in the reactive deposition of $\mathrm{NiO}$ on $\mathrm{Fe}$, the evaporation of metallic $\mathrm{Ni}$ is performed at a rate of about $1 \mathrm{ML} / \mathrm{min}$ in an oxygen background typically of $10^{-6}$ mbar. In these conditions, the ratio between the flux of oxygen molecules and of metal atoms impinging on the surface is about 45. The substrate is thus covered by its native oxide film before a significant amount of $\mathrm{Ni}$ is deposited.

Owing to the similar heat of formation and preparation procedures, $\mathrm{CoO} / \mathrm{Fe}(\mathrm{Fe} / \mathrm{CoO})$ heterostructures behave similarly to the $\mathrm{NiO} / \mathrm{Fe}(\mathrm{Fe} / \mathrm{NiO})$ systems. While metallic $\mathrm{Fe}$ deposited on the $\mathrm{CoO}(001)$ surface reacts forming a thin FeO layer [44] and a CoFe alloy [45], reactive deposition of $\mathrm{CoO}$ on the $\mathrm{Fe}(001)$ surface leads to the formation of thicker Fe oxides with higher oxidation states $[46,47]$.

An extensive substrate oxidation has been reported also in the case of the $\mathrm{TiO}_{2} / \mathrm{Fe}(001)$ interface [48]. Moreover, when the $\mathrm{TiO}_{2}$ growth is performed at $573 \mathrm{~K}$, a thick $\mathrm{Fe}_{3} \mathrm{O}_{4}$ layer covers the $\mathrm{TiO}_{2}$ film. Conversely, a limited amount of Fe oxide forms at the $\mathrm{Fe} / \mathrm{TiO}_{2}$ interface [49]. Upon thermal treatments in $\mathrm{UHV}$, Fe forms metal clusters which are almost completely covered by Ti suboxides [49].

An even richer phenomenology and somehow controversial results can be found in the case of interfaces formed between $\mathrm{MgO}$ and $\mathrm{Fe}(001)$. While most of the experimental works report on the formation of an atomically sharp $\mathrm{Fe} / \mathrm{MgO}$ interface [50-52], in line with the lower heat of formation of $\mathrm{MgO}$ with respect to that of Fe oxides (see Table 1), in some cases the presence of $\mathrm{Fe}$ oxide at the $\mathrm{Fe} / \mathrm{MgO}$ interface has been reported $[53,54]$. Concerning the $\mathrm{MgO} / \mathrm{Fe}$ interface, high 
quality $\mathrm{MgO}$ films can be obtained by direct evaporation of stoichiometric $\mathrm{MgO}[55,56]$, a procedure that allows avoiding the strong Fe oxidation induced by the oxygen atmosphere employed during reactive deposition [57]. Nevertheless, a substoichiometric $\mathrm{FeO}$ layer has been found to develop at the $\mathrm{MgO} / \mathrm{Fe}(001)$ interface [58].

\subsection{Interface structure}

The structural properties of $\mathrm{M} / \mathrm{O}$ and $\mathrm{O} / \mathrm{M}$ interfaces have been investigated by aiming the focus of the discussion mainly on three issues: (i) mesoscopic interface morphology, (ii) thermodynamic properties, described in terms of free energy surface densities, and (iii) lattice mismatch across the interface. Here, by "mesoscopic morphology" we mean the M/O (O/M) structure on a length scale of tens/hundreds of nanometers, including interface roughness, defect density, island size and distribution, etc. The mesoscopic morphology is, to a large extent, influenced by the quality of the substrate and by the specific preparation methods employed to grow the heterostructure. Surface and interface free energies determine instead the energetic balance of the heteroepitaxial system, driving its morphological evolution during the early stages of growth. Finally, lattice mismatch is directly connected with the microscopic details of the interface formation between materials characterized by different lattice constants. Its role is prominent at high coverages, since the elastic energy stored in the system increases linearly with the film thickness. In the following, for clarity sake, we will artificially try to isolate and discuss the importance of these phenomena in determining the final interface characteristics, however one should always keep in mind that they are intimately interwoven and cannot really be treated separately.

\subsubsection{Mesoscopic morphology}

Because of the high stability of oxide materials, metal deposition barely affects the mesoscopic morphology of their surfaces, while oxide layers deposited on metallic surfaces generally induce significant modifications of the substrate morphology at the nanometer scale. When oxide layers are stabilized on inert but mechanically soft substrates, such as, for instance, $\mathrm{Au}$ or $\mathrm{Ag}$, a remarkable surface etching is promoted by the overlayer. A widely investigated system in which such a process has been observed is the $\mathrm{NiO} / \mathrm{Ag}(001)$ interface, where the deposition of $\mathrm{NiO}$ induces the displacement of $\mathrm{Ag}$ atoms from the terraces. The resulting complex interface is characterized by the presence of vacancy islands on the substrate and $\mathrm{NiO}$ islands embedded in the topmost layers of the $\mathrm{Ag}(001)$ surface [59-65]. Oxide islands embedded in metallic substrates have been observed also for $\mathrm{MgO}[66,67]$ and $\mathrm{FeO}[68]$ nanolayers grown on $\mathrm{Ag}(001)$, as well as for $\mathrm{FeO}$ [69] and $\mathrm{CoO}$ [70] films deposited on $\mathrm{Au}(111)$.

A quasi-unidimensional etching of the substrate characterizes the growth of $\mathrm{CoO}$ onto stepped Pd surfaces [71]. Here, the decoration of step edges by $\mathrm{CoO}$ nanowires induces the ejection of a fraction of $\mathrm{Pd}$ atoms from the outer terrace, resulting in nanowires partially embedded in the upper terrace.
Fig. 2(a) displays a large-scale constant-current STM image of the $\operatorname{Pd}\left(\begin{array}{lll}1 & 1 & 23\end{array}\right)$ surface covered by an oxygen overlayer [71]. The substrate mesoscopic morphology is characterized by straight step edges, arranged periodically over the surface. The oxygen overlayer forms a $p(2 \times 2)$ pattern with respect to the substrate unit cell, as visible from the atomically resolved STM image shown in the inset of Fig. 2(a). After reactive deposition of $\mathrm{Co}, \mathrm{CoO}_{x}$ rows with a pronounced chemical contrast decorate the step edges. In Fig. 2(c) and (d) these $\mathrm{CoO}_{x}$ quasi-unidimensional nanostructures are delimited by dashed lines. In addition, rod-like structures decorating the $\mathrm{CoO}_{x}$ nanowires are visible, marked with arrows in Fig. 2(b) and ellipses in Fig. 2(d). These rods are arranged periodically along the direction parallel to the step edges, as evident from the Fast Fourier Transform of the STM image displayed in the inset of Fig. 2(b). STS data (not shown) reveal that the rod-like structures are composed by Pd atoms displaced from the substrate upper terrace [71].

Another important issue to be considered for understanding the $\mathrm{O} / \mathrm{M}$ interface morphology is represented by the substrate roughening induced by the oxygen background employed during reactive deposition. This phenomenon is particularly relevant when highly oxidizable metals are used as substrates (see Section 3), while for metallic substrates with a lower oxygen affinity the effect is less pronounced. However, a remarkable exception is provided by the case of $\mathrm{NiO}$ films stabilized on $\operatorname{Pd}(001)$ [72]. Despite Pd cannot be bulk-oxidized under UHV conditions [72], the post-oxidation of ultra-thin $\mathrm{Ni}$ films (thickness below two ML), deposited on the $\operatorname{Pd}(001)$ surface, promotes a considerable degree of substrate oxidation, preventing the stabilization of long-range ordered $\mathrm{NiO}$ overlayers. The promotion of $\mathrm{Pd}$ oxidation was ascribed to a lowering of the Pd work function induced by the $\mathrm{Ni}$ overlayer [72]. On the other hand, a negligible substrate oxidation and well ordered epitaxial $\mathrm{NiO}(100)$ layers are obtained if $\mathrm{Ni}$ deposition and post-oxidation cycles are performed on an oxygen pre-saturated Pd surface [72].

The roughening of the substrate can be also associated with a modification of the step/terrace morphology. When vicinal surfaces are employed as templates for the stabilization of unidimensional or quasi unidimensional oxide structures, oxygen-induced step bunching or faceting often occurs [73-75]. Step bunching generally has a detrimental effect on the surface mesoscopic order, since step coalescence destroys the periodic arrangement of the steps. An interesting case in which the oxide overlayer stabilizes the step-terrace morphology is represented by nanowires [76] and stripes [77] of $\mathrm{Mn}$ oxide grown on $\operatorname{Pd}\left(\begin{array}{lll}1 & 17\end{array}\right)$, a surface vicinal to $\operatorname{Pd}(001)$. After step decoration by Mn oxide wires, the step edges become straighter with respect to those of the oxide-free surface. At higher oxide coverages, a $c(4 \times 2)$ phase is stabilized on the terraces, and the surface is characterized by a sharp terracewidth distribution, for which the mean step-step separation increases from $23-24 \AA$, characteristic of the $\operatorname{Pd}\left(\begin{array}{lll}1 & 1 & 17\end{array}\right)$ surface, to $28-29 \AA$.

The mesoscopic order of the oxide film can be also affected by chemical reactions occurring at the $\mathrm{O} / \mathrm{M}$ interface. Pal et al. 
a

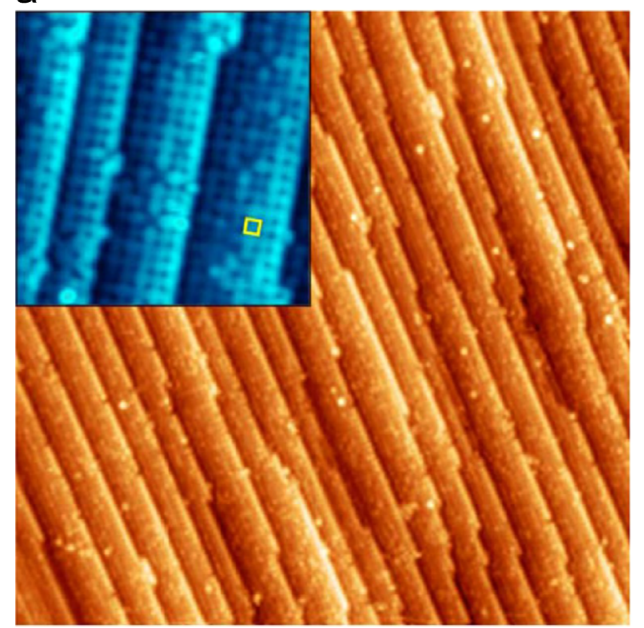

c

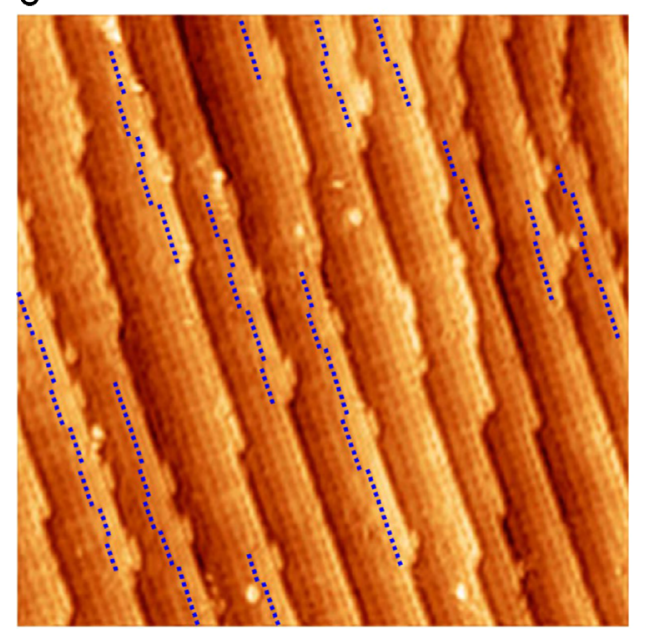

b

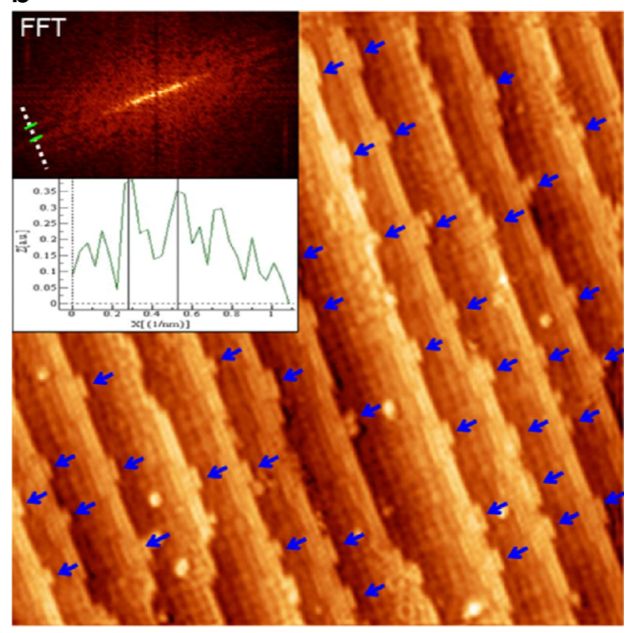

d

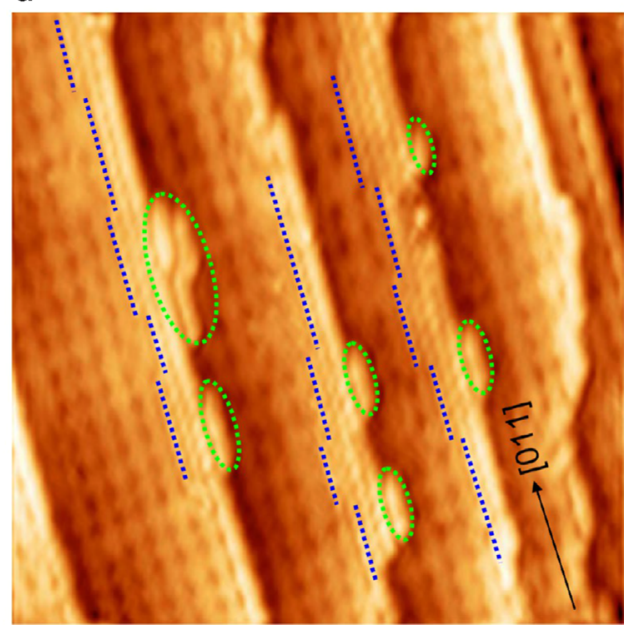

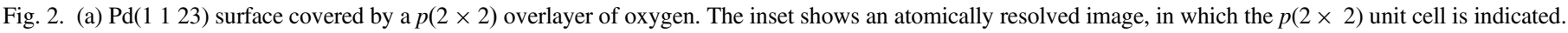

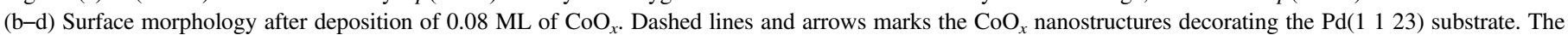

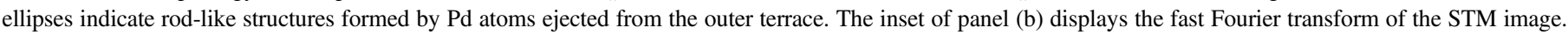

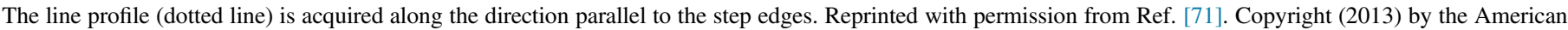
Chemical Society.

recently demonstrated the possibility of stabilizing wellordered $\mathrm{MgO}$ ultra-thin films on $\mathrm{Ag}(001)$ [78]. After hightemperature reactive deposition followed by a slow cooling, $\mathrm{MgO}$ terraces are obtained with a width reaching several tens of nanometers, while different preparation and post-growth conditions induce the nucleation of irregular monolayer islands or bi-layer islands [78]. Fig. 3(a)-(c) displays the morphology of sub-monolayer-thick $\mathrm{MgO}$ films stabilized on $\mathrm{Ag}(001)$, prepared by following different growth and post-growth protocols. The bottom part of each panel displays STM topographic images acquired at high voltage $(V \geq 3 \mathrm{~V})$, i.e. by tunneling into the $\mathrm{MgO}$ conduction band. In the upper part of the figure, the same surface regions are imaged at low bias $(V=1 \mathrm{~V})$, corresponding to an electron energy within the $\mathrm{MgO}$ band gap. Panel (a) corresponds to a $\mathrm{MgO}$ film grown at moderate temperature $(450 \mathrm{~K})$ and rapidly cooled at $200 \mathrm{~K}$. The constant current image displays relatively small islands, few nanometers wide, characterized by irregular borders. The topographic height of the islands [see Fig. 3(e)], measured at high bias voltage, is about $2 \AA$, corresponding to a thickness of a single atomic layer. In panel (b) the sample was obtained by a high-temperature $(773 \mathrm{~K})$ growth followed by a quick cooling to $200 \mathrm{~K}$. The increased growth temperature allows for the development of slightly larger and regular islands. However, in this case the islands are two-atomic-layer high, as testified by the topographic line shown in panel (e). Finally, the surface in panel (c) is obtained by growing the film at high temperature and slowly cooling the sample to $250 \mathrm{~K}$. In this case, large and monolayer-high $\mathrm{MgO}$ islands are obtained.

The formation of extended $\mathrm{MgO}$ monolayer terraces has been discussed in terms of interface chemistry and postgrowth kinetics [78,79]. On one hand, the high deposition temperature promotes the incorporation of oxygen atoms at the $\mathrm{MgO} / \mathrm{Ag}(001)$ interface. These possibly expand the substrate lattice and reduce the stress of the oxide film, improving its mesoscopic structure. On the other hand, the cooling rate determines whether the system can reach its low-temperature equilibrium state or is quenched into the configuration that is 


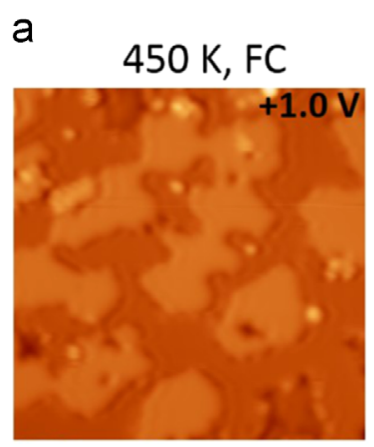

b
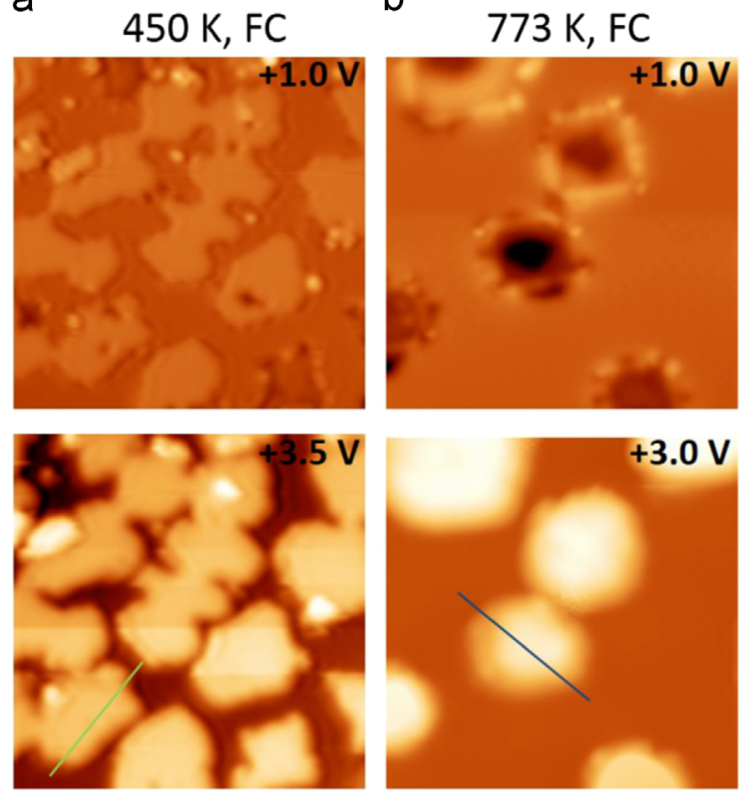

C $773 \mathrm{~K}, \mathrm{SC}$
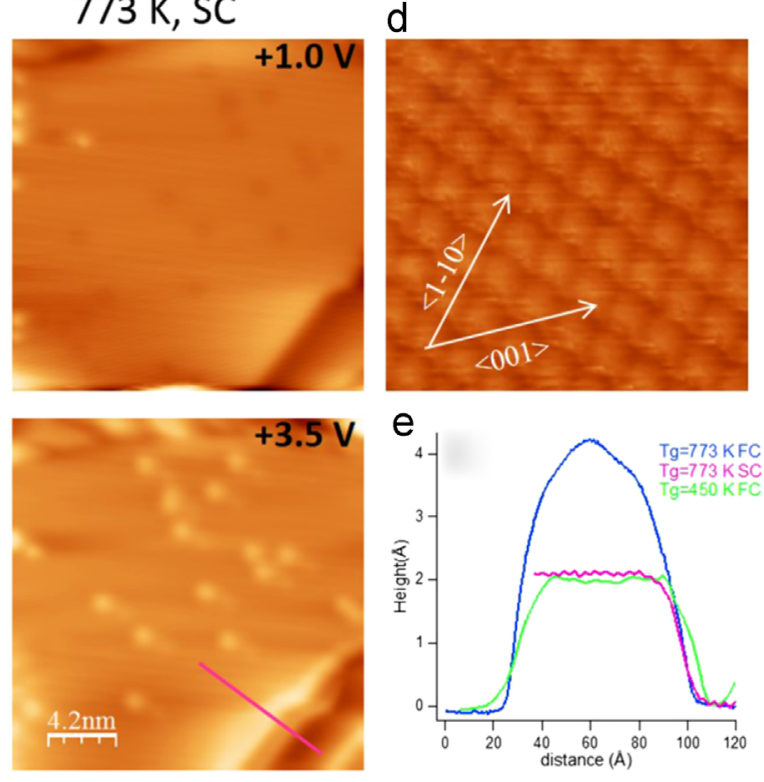

Fig. 3. (a-c) Top and bottom: STM images $\left(21 \times 21 \mathrm{~nm}^{2}\right)$ of $0.7 \mathrm{ML} \mathrm{MgO}$ stabilized on the $\mathrm{Ag}(001)$ surface at different growth conditions. If the sample cooling after $\mathrm{MgO}$ deposition is fast (FC), the film is characterized by the presence of small islands [panels (a) and (b), respectively], independently of the substrate temperature during $\mathrm{MgO}$ growth. Conversely, when the growth is performed at $773 \mathrm{~K}$ and the cooling rate is slow (SC), the film morphology displays extended atomically flat terraces [panel (c)]. (d) Atomically resolved image of the $\mathrm{Ag}(001)$ substrate. (e) Height profiles corresponding to the lines drawn in the bottom panels (a)-(c). Reprinted with permission from Ref. [78]. Copyright (2014) by the American Physical Society.

thermodynamically stable at the high temperature at which the deposition has been performed $[78,79]$.

Interface chemistry is also crucial for the nucleation of $\mathrm{FeO}$ islands on $\mathrm{Ru}(0001)$ [80]. At low oxygen pressure, monolayerhigh $\mathrm{FeO}$ islands cover the substrate, while bilayer islands of similar chemical composition nucleate when the growth is performed at higher background oxygen pressures. The nucleation process has been suggested to be driven by how the concentration of oxygen adsorbed on the $\mathrm{Ru}$ substrate changes as this oxygen is incorporated into the oxide film. More specifically, at low oxygen pressure, the oxygen concentration on $\mathrm{Ru}(0001)$ is limited and monolayer-high $\mathrm{FeO}$ islands can form. At higher oxygen pressure, monolayer growth is suppressed, leading to exclusive bilayer island nucleation, which is needed to remove oxygen atoms from the substrate [80].

\subsubsection{Energetic balance}

The energetic balance, driving the early stages of growth of either $\mathrm{M} / \mathrm{O}$ or $\mathrm{O} / \mathrm{M}$ interfaces, is governed by the surface free energy $\gamma$, defined as the energy per unit area required to create a surface starting from the bulk material. The modification of the surface free energy $\Delta \gamma$ induced by film deposition on a substrate can be written as $\Delta \gamma=\gamma_{\text {dep }}-\gamma_{\text {sub }}+\gamma_{\text {int }}$, where $\gamma_{\text {dep }}$ $\left(\gamma_{\text {sub }}\right)$ are the surface free energies of the deposit (substrate), while $\gamma_{\text {int }}$ is the interface energy per unit area. If $\Delta \gamma<0$, the formation of a wetting layer is energetically favored, while for $\Delta \gamma>0$ islanding is observed. If the interaction between the oxide and the metal is weak, the generally lower $\gamma$ values of oxide surfaces with respect to those characteristic of metal surfaces (see Table 2) steer two-dimensional growth of oxide
Table 2

Surface free energies per unit surface of common oxides and metals. The values concerning the metal surfaces are extracted from Ref. [81].

\begin{tabular}{ll}
\hline Oxide & $\gamma\left[\mathrm{J} / \mathrm{m}^{2}\right]$ \\
\hline $\mathrm{CoO}(001)[82]$ & 0.8 \\
$\mathrm{Fe}_{3} \mathrm{O}_{4}(001)[83]$ & 0.96 \\
$\mathrm{MgO}(001)[84]$ & 0.94 \\
$\mathrm{NiO}(001)[85]$ & 1.74 \\
$\mathrm{Al}_{2} \mathrm{O}_{3}(0001)[86]$ & 2.03 \\
$\mathrm{Cr}_{2} \mathrm{O}_{3}(0001)[87]$ & 3.1 \\
& \\
$\mathrm{Metal}$ & $\gamma\left[\mathrm{J} / \mathrm{m}^{2}\right]$ \\
\hline $\mathrm{Ag}(100)$ & 1.2 \\
$\mathrm{Au}(001)$ & 1.62 \\
$\mathrm{Fe}(001)$ & 2.222 \\
$\mathrm{Ni}(001)$ & 2.426 \\
$\mathrm{Co}(0001)$ & 2.775 \\
$\mathrm{Cr}(001)$ & 3.979 \\
\hline
\end{tabular}

films on metallic substrates, whereas islanding is observed in the case of metals deposited on oxide surfaces.

The chemical reactions occurring at the M/O interface may change the energetic balance thanks to the lowering of the interface energy term. A stronger adhesion between the metallic overlayer and the oxide surface promotes the formation of a continuous film. An example of chemically induced switching from three-dimensional to two-dimensional growth has been observed in the case of the $\mathrm{Co} / \mathrm{Al}_{2} \mathrm{O}_{3}(0001)$ interface [88]. Co films grown on the bare $\mathrm{Al}_{2} \mathrm{O}_{3}(0001)$ surface form three-dimensional clusters, while $\mathrm{Co}$ deposited on fullyhydroxylated $\mathrm{Al}_{2} \mathrm{O}_{3}(0001)$ wets the substrate [88]. The twodimensional growth is triggered by the chemical interaction of 
Co atoms with adjacent $\mathrm{OH}$ groups present on the hydroxylated surface, with the formation of $\mathrm{Co}^{2+}$ ions. The presence of these ions, strongly bound to the substrate, allows the development of smooth Co films.

Similar results were obtained for the $\mathrm{Cu} / \mathrm{Al}_{2} \mathrm{O}_{3}$ interface, which has been theoretically investigated by means of firstprinciples molecular dynamics simulations [89]. Cu nucleation on the hydroxylated $\mathrm{Al}_{2} \mathrm{O}_{3}$ surface results in the nucleation of single-layer islands composed by oxidized $\mathrm{Cu}$, while threedimensional growth is predicted on the anhydrous surface.

Interface smoothness can be improved also by direct spillover of oxygen from the oxide support, as demonstrated in the case of $\mathrm{Cr}$ films deposited on the $\mathrm{SrTiO}_{3}$ surface [90]. A threedimensional morphology develops after $\mathrm{Cr}$ deposition at room temperature, while annealing the film at high temperature induces a flattening of the $\mathrm{Cr}$ clusters, driven by $\mathrm{Cr}$ oxidation and by the consequent enhanced adhesion at the $\mathrm{M} / \mathrm{O}$ interface.

Another mechanism to promote adhesion at the $\mathrm{M} / \mathrm{O}$ interface can be represented by charge transfer, either from the metallic substrate on which the oxide is supported or from foreign atomic species dispersed in the oxide matrix. Gold deposited onto thin $\mathrm{CaO}$ films grown on $\mathrm{Mo}(001)$ forms twodimensional islands, whereas on thicker $\mathrm{CaO}$ films a threedimensional growth is observed. The two-dimensional growth is promoted by charge transfer from the underlying Mo substrate to the $\mathrm{Au} / \mathrm{CaO}$ interface [91]. Similar results have been found in the case of $\mathrm{Au}$ clusters deposited on $\mathrm{MgO}$ thin films supported on $\operatorname{Mo}(001)[92,93]$.

\subsubsection{Lattice matching}

The relevant parameter determining the elastic interaction between the epilayer and the substrate is the lattice mismatch, defined as $f=\frac{a_{\mathrm{o}}-a_{\mathrm{s}}}{a_{\mathrm{s}}}$, where $a_{\mathrm{o}}$ and $a_{\mathrm{s}}$ are the overlayer and substrate lattice constants, respectively. While the crystal structure of most metallic elements can be basically classified into three categories [i.e. the body centered cubic (bcc), face centered cubic (fcc) and hexagonal close packed (hcp) atomic arrangements], the oxidic compounds are characterized by a richer variety of crystal structures. Restricting the discussion to the cubic oxide crystal structures, the most common ones are fcc: rocksalt (where both anions and cations form fcc sublattices), spinel (only anions in fcc sites), and perovskite (anions and low-oxidation-state cations in fcc sublattices). In heterostructures composed by such fcc cubic oxides and fcc metals, the lattice mismatch $f$ for interfaces parallel to the (001) plane can be obtained by directly comparing the oxide and metal lattice constants $a_{\text {oxide }}$ and $a_{\text {metal }}$, respectively. On the other hand, for interfaces involving bcc metals, the oxide fcc sublattice should be rotated by $45^{\circ}$ around the [001] direction in order match the and the bcc lattice, so that the $f$ value should be estimated by comparing $a_{\text {metal }}$ and $a_{\text {oxide }} / \sqrt{2}$ (see Table 3 for a list of lattice mismatch values for bcc $\mathrm{Fe}(001)$ with respect to the (001) surface of several oxides possessing a cubic unit cell).

The accumulation of elastic energy can be released by the growth of three dimensional islands on top of the wetting layer (Stransky-Krastanov growth) and/or by means of plastic
Table 3

Mismatch $f_{\mathrm{Fe}(001)}=\frac{a_{\text {oxygen }}-a_{\mathrm{Fe}(001)}}{a_{\mathrm{Fe}(001)}}$ between bcc $\mathrm{Fe}(001)$ and common cubic oxides. Column $a_{\text {oxide }}$ lists the values of the bulk oxide lattice parameter, while $a_{\text {oxygen }}$ indicates the size of the unit cell of the square sublattice formed by oxygen atoms exposed at the oxide (001) surface. The lattice constant of bcc $\mathrm{Fe}$ is $a_{\mathrm{Fe}}=287 \mathrm{pm}$.

\begin{tabular}{llllc}
\hline Oxide & Structure & $a_{\text {oxide }}[\mathrm{pm}]$ & $a_{\text {oxygen }}[\mathrm{pm}]$ & $f_{\mathrm{Fe}(001)}(\%)$ \\
\hline $\mathrm{CoO}[94]$ & Rocksalt & 426.1 & 301.3 & 5 \\
$\mathrm{Co}_{3} \mathrm{O}_{4}[95]$ & Spinel & 808.4 & 285.8 & -0.4 \\
$\mathrm{NiO}[96]$ & Rocksalt & 417.6 & 295.3 & 2.9 \\
$\mathrm{FeO}[97]$ & Rocksalt & 417.2 & 295.0 & 2.8 \\
$\mathrm{Fe}_{3} \mathrm{O}_{4}[98]$ & Inverse spinel & 839.4 & 296.8 & 3.4 \\
$\mathrm{MgO}_{[99]}$ & Rocksalt & 421.0 & 297.7 & 3.7 \\
$\mathrm{SrTiO}_{3}[100]$ & Perovskite & 390.5 & 276.1 & -3.8 \\
\hline
\end{tabular}

deformations, i.e. by involving the formation of misfit dislocations, found both in M/O [101] and O/M interfaces [102,103]. The dislocations often arrange in an ordered network, developing at correspondence with the coincidence-site lattice between the overlayer and the substrate. The coincidence-site lattice is defined as the superlattice formed by Bravais vectors that the two primitive ones have in common, with a period given by $a_{\mathrm{o}} /|f|$.

The partial modification of the oxide stoichiometry in proximity of the interface region plays an important role in accommodating strain at the metal-oxide interface. For instance, the presence of reduced species at the $\mathrm{O} / \mathrm{M}$ interfaces has been associated with strain release [104]. This circumstance can be understood by considering that oxides are generally stiffer than metals, thereby the presence of reduced species at the interface can lower the elastic energy stored in the film. In the case of one-layer-thick oxides supported on metallic substrates, also cations deficient films have been observed. Pd(001)-supported transition metal (TM) oxides, where TM is $\mathrm{Ni}$ [105], Mn [106], and Co [107], in the monolayer limit form a $c(4 \times 2)$ phase, with $\mathrm{TM}_{3} \mathrm{O}_{4}$ formal stoichiometry. The $c(4 \times 2)$ phase is obtained by removing one quarter of TM cations from the (001) surface of the stoichiometric rocksalt monoxide. The formation of cationic vacancies partially releases the large lattice mismatch existing between the $\operatorname{Pd}(001)$ substrate and the bulk TMO rocksalt (001) surface, allowing for the development of ordered oxide wetting layers [107].

Besides the partial modification of the oxide stoichiometry, the development of mixed phases has also been observed and interpreted as an efficient mechanism to release the strain, as in the case of $\mathrm{CaO}$ films grown on $\mathrm{Mo}$ [108]. The (001) surface of the rocksalt $\mathrm{CaO}$ is symmetry-matched with the (001) surface of bcc $\mathrm{Mo}(001)$, but the lattice mismatch between the overlayer and the substrate is substantial $(+8.1 \%)$. When $\mathrm{CaO}$ films are grown on $\mathrm{Mo}(001)$ at room temperature, the resulting film is found to be amorphous, while upon annealing the film crystallizes and long-range order is achieved. It turns out that the large misfit is accommodated by a change of the stoichiometric composition at the interface, thanks to the incorporation of Mo ions into the $\mathrm{CaO}$ lattice (see Fig. 4). The diffusion of 


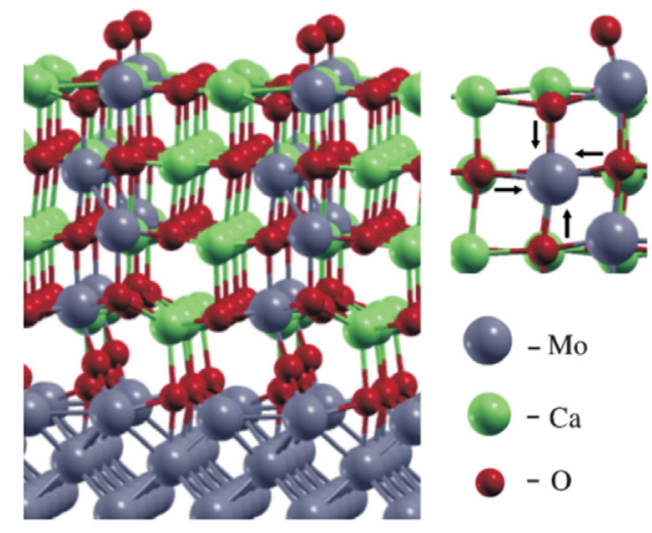

Fig. 4. Proposed model of the reactive $\mathrm{CaO} / \mathrm{Mo}$ interface, for which the ternary $\mathrm{Ca}-\mathrm{Mo}-\mathrm{O}$ phase forming at the oxide/metal boundary allows an efficient strain release. The right panel displays the inward relaxation of $\mathrm{O}$ ions next to a Mo impurity. Reprinted with permission from Ref. [109]. Copyright (2011) by the American Physical Society.

Mo atoms from the substrate allows the stabilization of a ternary $\mathrm{Ca}_{3} \mathrm{MoO}_{4}$ oxide phase, which possesses an improved lattice matching with the $\mathrm{Mo}(001)$ substrate [109].

Strictly speaking, the concept of lattice mismatch applies only when the substrate and the overlayer involved in the interface formation possess the same two-dimensional symmetry. Although this is often the case, many exceptions to this rule can be found in the literature, in particular for $\mathrm{O} / \mathrm{M}$ interfaces. A large number of experimental works report on the stabilization of oxide films growing with crystallographic orientations that do not follow the substrate symmetry. $\mathrm{FeO}(111)$ layers, characterized by an hexagonal surface symmetry, have been stabilized on square substrates such as $\operatorname{Pt}(001)$ [110] and $\operatorname{Ag}(001)$ [111,68]. Under specific preparation conditions, Co oxide on $\operatorname{Ir}(001)$ [112,113] and Mn oxide on $\operatorname{Pd}(001)$ [114] have been found to develop with the (111) orientation, notwithstanding the square symmetry of the substrate. The reverse case, i.e. squared oxide overlayers on hexagonal metallic substrates, has also been observed as in the case of $\mathrm{MgO}(001)$ stabilized on $\mathrm{Au}$ (111) [115]. In these symmetry-mismatched systems, coincidence lattice structures (or moiré patterns) are formed, characterized by periodic long range modulations of the oxide overlayer. On the other hand, similar symmetrymismatched $\mathrm{M} / \mathrm{O}$ interfaces have been rarely observed [91], because metallic deposits tend to form threedimensional clusters on oxide surfaces, hampering the development of long range moiré superstructures.

The interface chemistry can greatly influence the crystallographic orientation of oxide layers stabilized on metallic substrates, as demonstrated in the case of Co oxide nanofilms grown on the $\operatorname{Ir}(001)$ substrate [116]. CoO grows on the bare $\operatorname{Ir}(001)$ surface exposing the (111) surface, but the crystallographic orientation can be switched to (001) by introducing a thin Co buffer layer between the oxide and the substrate. Fig. 5(a) and (b) displays the LEED pattern and the STM topography of a single-layer defective Co oxide stabilized onto a wetting layer of Co covering the $\operatorname{Ir}(001)$ substrate. Both the LEED pattern [panel (a)] and the STM image [panel (b)] show that the Co oxide overlayer forms a $c(4 \times 2)$ superstructure with respect to the substrate lattice, consistent with a formal stoichiometry of $\mathrm{Co}_{3} \mathrm{O}_{4}$. When the $c(4 \times 2) \mathrm{Co}_{3} \mathrm{O}_{4} / \mathrm{Co} / \mathrm{Ir}$ (001) system is further oxidized, $\mathrm{CoO}(001)$ islands develop on the surface [panels (d) and (e)]. In the LEED pattern [panel (c)] the $c(4 \times 2)$ spots have disappeared and a moiré pattern with a periodicity of about $(12 \times 12)$ is visible, arising from the superposition of diffracted electrons from the $\mathrm{CoO}(001)$ overlayer and from the substrate. Panels (f) and ( $g$ ) display the fourfold symmetric LEED pattern and the STM image obtained after further reactive growth of Co. Both measurements are compatible with the development of a $\mathrm{CoO}(001)$ film. Conversely, $\mathrm{CoO}$ grown on bare $\operatorname{Ir}(001)$ exposes the (111) face, as testified by the LEED pattern and the atomically-resolved STM images displayed in panels (h) and (i), respectively.

Being the Co layer pseudomorphic to the $\operatorname{Ir}(001)$ substrate, $f$ is nominally the same for both the $\mathrm{CoO} / \operatorname{Ir}(001)$ and $\mathrm{CoO} / \mathrm{Co} / \mathrm{Ir}(001)$ epitaxial systems, indicating that the driving force determining the crystal growth orientation resides in the interface chemistry. In particular, the development of the $c(4 \times 2)$ superstructure, related to the presence of cation vacancies, has been suggested to be the key for the stabilization of $\mathrm{CoO}(001)$ [116]. A similar effect was recently observed for iron oxide grown on $\operatorname{Pt}(001)$, where $\mathrm{Fe}_{3} \mathrm{O}_{4}(111)$ films develop on the bare $\mathrm{Pt}(100)$ substrate [117], while the oxide grows exposing the (001) face if a $\mathrm{Fe}$ buffer layer is deposited on the $\operatorname{Pt}(001)$ surface before oxide deposition [117,118].

\subsection{Band alignment across the interface}

As a result of the markedly different band structure of insulators and metals, a discontinuity on the electronic energy scale is established at the metal/insulator heterojunction, leading to the development of an energetic barrier for the electrons (or holes) traveling across the interface. The main effect of such a barrier is to produce an asymmetric bias dependence of the current flowing across the interface, leading to the so-called rectifying behavior of the heterojunction. These issues have been extensively discussed in the field of metal/semiconductor heterophases [119], but the increasing interest in all-oxide electronics has boosted a considerable effort towards the study of metal-oxide electronic coupling. In this field, besides the research aiming to achieve an improvement of the carrier mobility, a crucial issue is to find the right metal-oxide combination to obtain ohmic and low-resistance contacts.

In the original model proposed by Schottky, the $p$-type barrier can be written as $\phi_{p}=I P-\phi_{\mathrm{m}}$, where $I P$ and $\phi_{\mathrm{m}}$ are the insulator ionization potential and the metal work function, respectively. The $n$-type barrier is given by $\phi_{n}=\phi_{\mathrm{m}}-\chi$, where $\chi=I P-E_{\mathrm{g}}$ is the insulator electron affinity, defined as the difference between $I P$ and the energy gap $\mathrm{E}_{g}$. However, this model needs to be refined to take into account the presence of interfacial states that can efficiently screen the insulator. The model should thus include an additional term in the Schottky 



Fig. 5. (a and b) LEED pattern and STM topography of the $c(4 \times 2)$ superstructure developing on the Co/Ir(001) system. (c-e) Reciprocal and real space lattices of the surface obtained after oxidation of the $c(4 \times 2)$ phase. (f and g) Development of $\mathrm{CoO}(001)$ upon reactive deposition of Co on the $c(4 \times 2)$ surface. (h and i) LEED pattern and STM topography of $\mathrm{CoO}(111)$, developing on the bare $\operatorname{Ir}(001)$ substrate. Reprinted with permission from Ref. [116]. Copyright (2012) by the American Physical Society. 


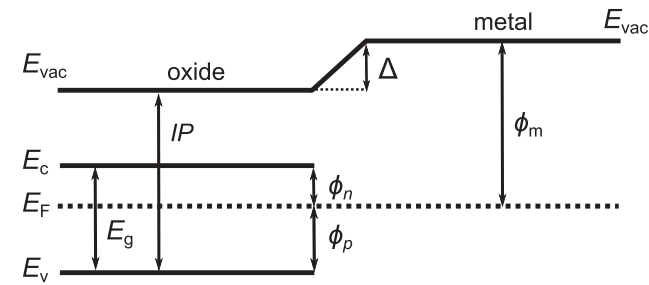

Fig. 6. Simplified scheme of the energy level alignment at the insulator/metal interface. The insulator is considered in flat band conditions. $I P=$ oxide ionization potential, $E_{\mathrm{F}}=$ Fermi level, $E_{\mathrm{g}}=$ energy gap, $E_{\mathrm{vac}}=$ vacuum level, $E_{\mathrm{c}}=$ oxide conduction band minimum, $E_{\mathrm{v}}=$ oxide valence band maximum, $\phi_{\mathrm{m}}=$ metal work function, $\phi_{n}=n$-type barrier, $\phi_{p}=p$-type barrier, $\Delta=$ interface dipole. barrier, related to the presence of an interface dipole $\Delta$ (see Fig. 6). In this case the $p$-type and $n$-type Schottky barriers are given by $\phi_{p}=I P+\Delta-\phi_{\mathrm{m}}$ and $\phi_{n}=\phi_{\mathrm{m}}-(\Delta+\chi)$, respectively. $\Delta$ is generally related to the presence of electronic states in the insulator gap, either intrinsic or induced by the presence of the metal. Due to the intimate relation between the interface chemistry and the lining up of the insulator/metal bands, the chemical interactions of metal atoms with the oxide surface are expected to play a prominent role in the determination of the Schottky barrier.

A noteworthy situation where such an influence has been found for the $\mathrm{M} / \mathrm{O}$ interface is the case of the

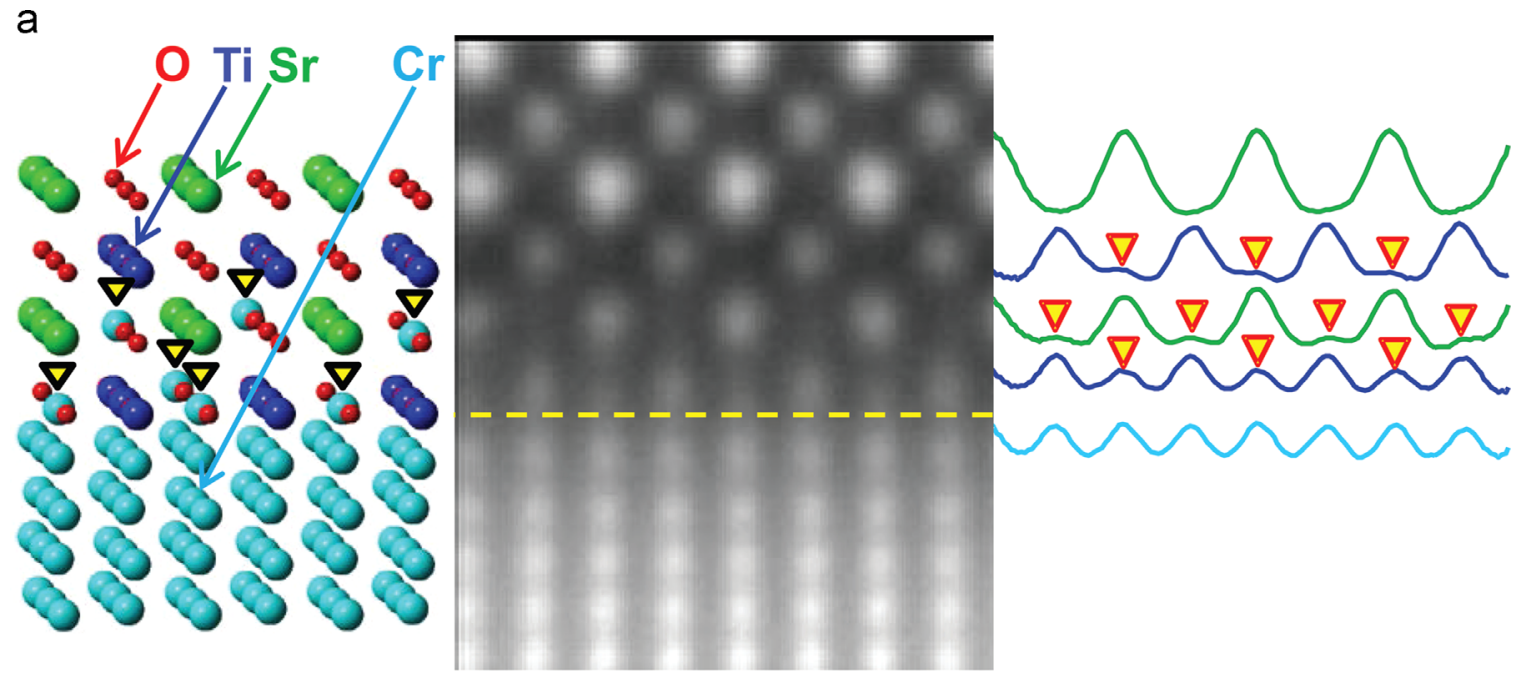

b
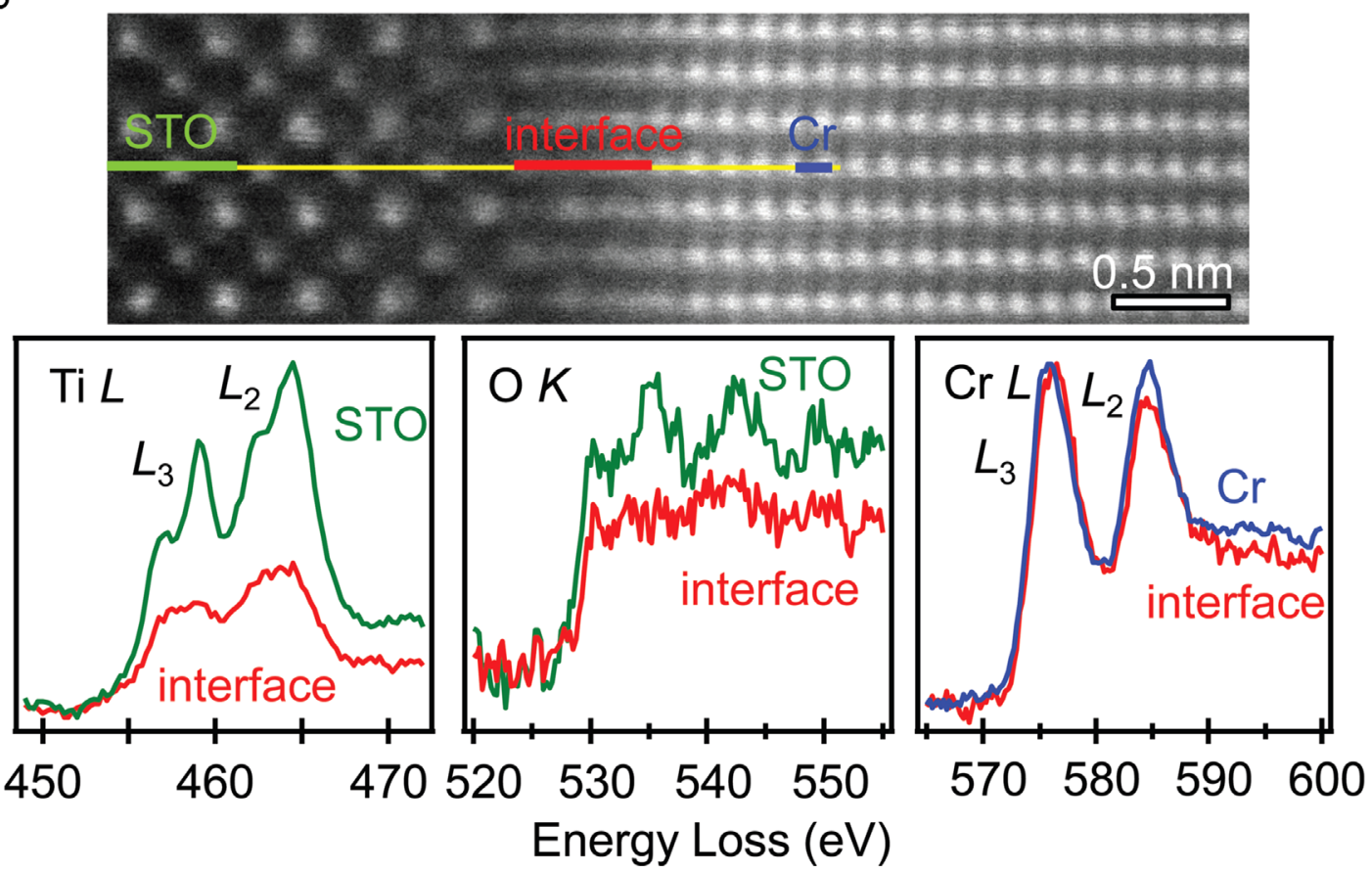

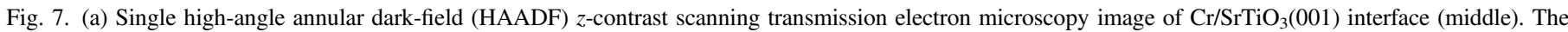

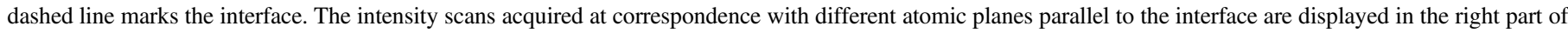

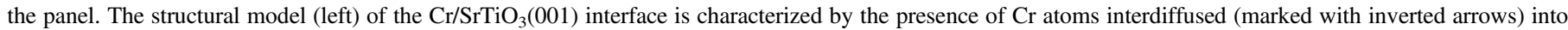

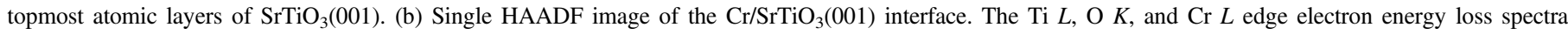

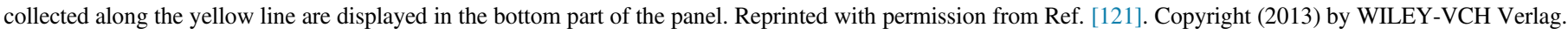
(For interpretation of the references to color in this figure caption, the reader is referred to the web version of this paper.) 
$\mathrm{M} / \mathrm{SrTiO}_{3}(001)$ heterojunction. While the interface between a noble metal such as $\mathrm{Au}$ and $\mathrm{SrTiO}_{3}(001)$ is characterized by the formation of a Schottky barrier [120], the $\mathrm{Cr} / \mathrm{SrTiO}_{3}(001)$ contact exhibits a low resistance and is ohmic $[121,122]$. This stems from the fact that the epitaxial $\mathrm{Cr}$ films do not form an abrupt interface with the $\mathrm{SrTiO}_{3}(001)$ substrate, but a fraction of $\mathrm{Cr}$ atoms diffuses into it, occupying interstitial sites within the first few atomic planes. At the same time, the $\mathrm{Cr}$ atoms become ionized and strongly dope the near-interface region, forcing $E_{\mathrm{F}}$ above the conduction band minimum.

Fig. 7(a) (middle) displays a Z-contrast scanning transmission electron microscopy (STEM) image of the $\mathrm{Cr} / \mathrm{SrTiO}_{3}(001)$ interface [121]. Intensity profiles acquired on different atomic layers [right panel of Fig. 7(a)] indicate the presence of foreign atoms (marked with inverted arrows), located in the $\mathrm{SrTiO}_{3}(001)$ interstitial sites. The foreign species are identified as $\mathrm{Cr}$ atoms, based on $\mathrm{Cr} L$-edge electron energy loss spectroscopy spectra measured at selected points with a transmission electron microscope along lines perpendicular to the interface [Fig. 7(b)]. A schematic structural model, showing $\mathrm{Cr}$ atoms interdiffused within the first two atomic layer of the $\mathrm{SrTiO}_{3}(001)$ substrate, is displayed in the left part of Fig. 7(a).

Lenser et al. obtained similar results by comparing the transport properties of $\mathrm{M} / \mathrm{SrTiO}_{3}(001)$ heterojunctions, where the metallic electrode was either the noble metal $\mathrm{Pt}$ or the highly reactive metal $\mathrm{Ti}$ [123]. In the former case, the junction was found to exhibit rectifying properties, whereas in the latter the interface exhibits flat-band electronic structure and symmetric $I(V)$ characteristics.

Turning to the $\mathrm{O} / \mathrm{M}$ case, an example of chemically induced modifications of the Schottky barrier can be recognized in the $\mathrm{MgO} / \mathrm{Ag}(001)$ interface obtained by $\mathrm{Mg}$ reactive deposition $[124,125]$. Depending on the oxygen partial pressure and the substrate temperature employed during the reactive deposition of ultra-thin Mg oxide films, the $p$-type Schottky barrier has been tuned over $0.7 \mathrm{eV}$ [125]. These remarkable variations of the Schottky barrier were ascribed to the decrease of the silver work function induced by the $\mathrm{Mg}$ enrichment of the substrate surface region (associated to the formation of a $\mathrm{Mg}-\mathrm{Ag}$ alloy) when $\mathrm{Mg}$ is evaporated in oxygen-poor conditions or at high substrate temperatures $[125,126]$.

Yoshitake et al. studied the relation between the chemical composition and the band alignment in $\mathrm{Al}_{2} \mathrm{O}_{3} / \mathrm{M}$ interfaces, where the metallic substrates were pure metals, $\mathrm{Cu}(111)$ and $\mathrm{Ni}(111)$, or Al-based intermetallic compounds, $\mathrm{Cu}-9$ at $\% \mathrm{Al}$ (111) and $\mathrm{NiAl}(110)$ [127]. The $\mathrm{Al}_{2} \mathrm{O}_{3} / \mathrm{Cu}(111)\left[\mathrm{Al}_{2} \mathrm{O}_{3} / \mathrm{Cu}-9\right.$ at $\% \mathrm{Al}(111)]$ and $\mathrm{Al}_{2} \mathrm{O}_{3} / \mathrm{Ni}(111)\left[\mathrm{Al}_{2} \mathrm{O}_{3} / \mathrm{NiAl}(110)\right]$ interfaces have been found to be O-terminated [Al-terminated]. The $p$-type Schottky barrier depends on the interface composition, being larger when the interfaces are Al-terminated. The band offset for the $\mathrm{Al}_{2} \mathrm{O}_{3} / \mathrm{NiAl}(110)\left[\mathrm{Al}_{2} \mathrm{O}_{3} / \mathrm{Cu}-9\right.$ at $\left.\% \mathrm{Al}(111)\right]$ interface is $1.7 \mathrm{eV}[1.2 \mathrm{eV}]$ larger than for the $\mathrm{Al}_{2} \mathrm{O}_{3} / \mathrm{Ni}(111)$ $\left[\mathrm{Al}_{2} \mathrm{O}_{3} / \mathrm{Cu}(111)\right]$ interface.

The origin of the different band offset observed in Al- and O-terminated $\mathrm{Al}_{2} \mathrm{O}_{3} / \mathrm{M}$ interfaces has been discussed in
Ref. [128], where the $\mathrm{Al}_{2} \mathrm{O}_{3} / \mathrm{Cu}-9$ at $\% \mathrm{Al}(111)$ case was analyzed. For an Al-terminated interface, $E_{\mathrm{F}}$ aligns with the $\mathrm{Al}_{2} \mathrm{O}_{3}$ gap states induced by the presence of $\mathrm{Al}$ dangling bonds. The gap states are located slightly above the middle of the $\mathrm{Al}_{2} \mathrm{O}_{3}$ gap. Therefore, the maximum of the $\mathrm{O} 2 p$-derived valence band of $\mathrm{Al}_{2} \mathrm{O}_{3}$ is approximately $4 \mathrm{eV}$ below $E_{\mathrm{F}}$, resulting in a $p$-type Schottky barrier of about $4 \mathrm{eV}$. On the other hand, when the interface is established between the Oterminated alumina and $\mathrm{Cu}$, a strong hybridization between the $\mathrm{Cu} 3 d$ and $\mathrm{O} 2 p$ electronic states occurs; this places the $\mathrm{O} 2 p$ orbitals of the alumina valence band near the $\mathrm{Cu} 3 d$ levels. Since $\mathrm{Cu}$ possesses a high $3 d$ density of states approximately $2 \mathrm{eV}$ below $E_{\mathrm{F}}$, the $p$-type Schottky barrier falls to the $2 \mathrm{eV}$ range.

\subsection{Magnetic coupling}

Interfaces between oxides and metals find a widespread application in the field of magnetic layered structures $[12,129,130]$. Since the magnetic coupling in such systems is mainly driven by the interactions occurring at the interface, it is of paramount importance to have a full control of the interfacial chemical composition. Indeed, the local chemical environment directly influences the spin ordering, since often the oxidic compounds possess a different magnetic order with respect to that of the parent metallic crystals (see Table 4). Magnetic moments are ferromagnetically coupled in $\mathrm{Fe}$, Co and $\mathrm{Ni}$ crystals, while the spin structure of the respective rocksalt monoxides is characterized by ferromagnetically coordinated cations with adjacent $\{111\}$ planes coupled antiferromagnetically to each other. Conversely, $\mathrm{Cr}$ bcc crystals exhibit an antiferromagnetic order, while $\mathrm{CrO}_{2}$ is a halfmetallic ferromagnet.

Because of the different chemical composition of the nominally symmetric $\mathrm{M} / \mathrm{O}$ and $\mathrm{O} / \mathrm{M}$ heterosystems (see Section 2.1), also the resulting interfacial magnetic order is considerably different. An example can be found again in $\mathrm{Fe} / \mathrm{NiO}(\mathrm{NiO} / \mathrm{Fe})$ heterostructures, where either a thin antiferromagnetic $\mathrm{FeO}$ oxide [139] or a ferrimagnetic $\mathrm{Fe}_{3} \mathrm{O}_{4}[41,43]$ are stabilized at $\mathrm{Fe} / \mathrm{NiO}$ and $\mathrm{NiO} / \mathrm{Fe}$ interfaces, respectively. In both cases, the magnetic coupling

Table 4

Magnetic order and magnetic moments $(m)$ expressed in Bohr magnetons $\left(\mu_{\mathrm{B}}\right)$ for magnetic transition metals and corresponding oxide compounds.

\begin{tabular}{lll}
\hline Material & Magnetic order & $m\left(\mu_{\mathrm{B}}\right)$ \\
\hline $\mathrm{Cr}$ & Antiferromagnetic & $0.59[131]$ \\
$\mathrm{Cr}_{2} \mathrm{O}_{3}$ & Antiferromagnetic & $2.48[132]$ \\
$\mathrm{CrO}_{2}$ & Ferromagnetic & $2[133]$ \\
$\mathrm{Fe}$ & Ferromagnetic & $2.23[134]$ \\
$\mathrm{FeO}$ & Antiferromagnetic & $3.32[135]$ \\
$\mathrm{Fe}_{3} \mathrm{O}_{4}$ & Ferrimagnetic & $5\left(\mathrm{Fe}^{3+}\right), 4\left(\mathrm{Fe}^{2+}\right)[136,137]$ \\
$\mathrm{Co}$ & Ferromagnetic & $1.67[134]$ \\
$\mathrm{CoO}$ & Antiferromagnetic & $3.8[135]$ \\
$\mathrm{Ni}$ & Ferromagnetic & $0.59[134]$ \\
$\mathrm{NiO}$ & Antiferromagnetic & $1.9[138]$ \\
\hline
\end{tabular}


between $\mathrm{Fe}$ and $\mathrm{NiO}$ is not direct, but mediated by the $\mathrm{Fe}$ oxide layer in between.

A widely investigated magnetic phenomenon occurring at ferromagnetic metal-antiferromagnetic oxide interfaces is the exchange bias effect. First discovered in 1956 by Meiklejohn and Bean during the study of Co particles embedded in their native antiferromagnetic oxide [140], exchange bias is a general phenomenon occurring in coupled ferromagnetic-antiferromagnetic heterostructures. When the system is cooled through the Néel temperature of the antiferromagnet, unidirectional magnetic anisotropy is induced in the ferromagnetic layer, resulting in a shift of its hysteresis loop. Various theoretical models have been proposed in order to explain the exchange bias effect, many of them involving the presence of uncompensated spins at the interface between the ferromagnet and the antiferromagnet. The atomic spin structure is greatly influenced by the chemical environment, therefore the reactions occurring at the interface are expected to directly influence the number of uncompensated spins. Moreover, since structural defects are responsible for the development of magnetically frustrated regions [141], the chemical composition can also indirectly influence the interface magnetic order through structural modifications at mesoscopic scales.

For instance, Ohldag et al. correlated the presence of uncompensated spins at the $\mathrm{Co} / \mathrm{NiO}$ interface to the stabilization of an ultra-thin $\mathrm{CoNiO}_{x}$ layer, formed after the Co deposition through reduction of the $\mathrm{NiO}$ surface [142]. Bali et al. studied the influence of interfacial $\mathrm{Fe}$ oxide on the magnetic properties of $\mathrm{CoO} / \mathrm{Fe}$-wedged/ $\mathrm{Ag}(001)$ samples [47]. Upon $\mathrm{CoO}$ growth on $\mathrm{Fe}$ films of different thickness (4-16 ML), the interface is found to be characterized by the presence of a $\mathrm{Fe}$ oxide layer composed by $\mathrm{Fe}_{2} \mathrm{O}_{3}$ and $\mathrm{Fe}_{3} \mathrm{O}_{4}$, with the amount of the interfacial $\mathrm{Fe}$-oxide decreasing with increasing $\mathrm{Fe}$ film thickness and saturating for Fe films above $8 \mathrm{ML}$. The authors found a correlation between the thickness of the $\mathrm{Fe}$ oxide layer and the difference in exchange bias field induced by magnetic or zero field cooling, assigned to the evolution of the interface roughness. Młyńczak et al. recently studied the relation between the interfacial $\mathrm{Fe}$ oxidation and the exchange bias in $\mathrm{CoO} / \mathrm{Fe}$ samples grown on $\mathrm{MgO}$ [143]. Comparing samples characterized by different amounts of Fe oxides at the interface, it turned out that the shift of the hysteresis loop is related to the interface composition, being larger for the overoxidized $\mathrm{Fe}(001)$ substrates.

The chemical interactions occurring at the interface can induce exchange bias even in cases in which the ferromagnet is coupled with an oxide that does not display any magnetic order. Fan et al. studied the magnetic behavior of $\mathrm{Fe} / \mathrm{MgO}$, finding that $\mathrm{FeO}$ patches formed at the $\mathrm{Fe} / \mathrm{MgO}$ interface can act as an antiferromagnetic pinning layer, inducing a pronounced exchange bias confined to the interface magnetization [144]. Moreover, the authors demonstrated also the possibility to tune the magnitude of the exchange bias by changing the interface oxygen concentration.

\subsection{Tailoring the interface structure: buffer layers}

A versatile route to tailor the properties of both $\mathrm{M} / \mathrm{O}$ and $\mathrm{O} / \mathrm{M}$ interfaces is to deposit a different metallic or oxide thin buffer layer between the substrate and the overlayer, obtaining the different heterostructures $\left(\mathrm{M} / \mathrm{O}_{a} / \mathrm{O}_{b}, \mathrm{O} / \mathrm{M}_{a} / \mathrm{M}_{b}, \mathrm{M}_{a} / \mathrm{M}_{b} / \mathrm{O}\right.$, and $\left.\mathrm{O}_{a} / \mathrm{O}_{b} / \mathrm{M}\right)$ schematically displayed in Fig. 8. Buffer layers can be exploited to prevent unwanted chemical reactions at the interface or improve the interface morphology.

For what concerns $\mathrm{M} / \mathrm{O}_{a} / \mathrm{O}_{b}$ heterostructures, Allegretti et al. employed an oxide buffer layer in order to control the interface chemistry, finding that a sharp boundary between a ferromagnetic metal and an antiferromagnetic oxide can be obtained by inserting a thin $\mathrm{CoO}$ buffer layer between a $\mathrm{NiO}$ (100) surface and a Co film. XPS spectra reveal that the presence of a 1-2 ML thick $\mathrm{CoO}$ buffer layer, covering the $\mathrm{NiO}(001)$ surface, prevents its reduction when a submonolayer Co film is deposited [145]. A similar strategy was followed by Masi et al. who investigated, by means of XPS and LEED, the effect of $\mathrm{MgO}$ buffer layers deposited on $\mathrm{NiO}$ prior to Fe growth [146], finding an almost complete suppression of the $\mathrm{NiO}$ reduction characterizing the $\mathrm{Fe} / \mathrm{NiO}$ interface.

Considering $\mathrm{O} / \mathrm{M}_{a} / \mathrm{M}_{b}$ interfaces, metallic buffer layers can be exploited in order to stabilize high quality $\mathrm{O} / \mathrm{M}$ interfaces between a metal and its native oxide in those cases in which the respective crystal structures are markedly different in terms of lattice mismatch or crystal symmetry. For instance, rocksalt $\mathrm{NiO}$ possesses a large lattice mismatch with respect to fcc $\mathrm{Ni}$ [147], while thin Ni films grow pseudomorphic on $\mathrm{Fe}(001)$, i.e. with the same in-plane lattice constant of the $\mathrm{Fe}(001)$ surface [148-150], leading to a good lattice matching with $\mathrm{NiO}$ (see Table 3). The crystal structure of bulk Co is hcp, symmetrymismatched with respect to the rocksalt structured $\mathrm{CoO}$, while high quality tetragonally distorted body-centered-cubic Co films stabilized on $\mathrm{Fe}(001)$ [151-153] can be used as a template for obtaining epitaxial $\mathrm{CoO}$ layers $[154,155] . \mathrm{Mg}$ (whose bulk crystal structure is hcp) has been stabilized in the cubic phase on $\mathrm{Fe}(001)$ and then subsequently oxidized to obtain $\mathrm{MgO}$ [156]. Finally, a metallic buffer layer can also protect the substrate from oxidation during oxide deposition.

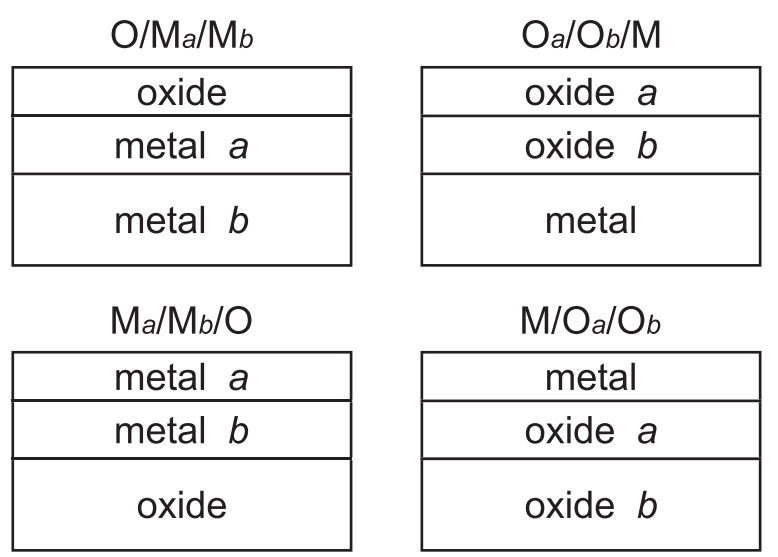

Fig. 8. Schematic representation of the three-layered structures containing a different metallic or oxide buffer layer across the $\mathrm{M} / \mathrm{O}$ and $\mathrm{O} / \mathrm{M}$ interface. 
Following this line, it has been shown that a thin Mg buffer layer prevents substrate oxidation when $\mathrm{MgO}$ layers are deposited on $\mathrm{CoFeB}$ [157].

As for $\mathrm{O}_{a} / \mathrm{O}_{b} / \mathrm{M}$ layered system, Xue et al. studied the effect of a Fe oxide buffer layers covering a $\mathrm{Mo}(110)$ substrate for the growth of polar $\mathrm{NiO}(111)$ films. Fe oxide buffer layers improve the $\mathrm{NiO}(111)$ growth with respect to the direct growth on the pristine $\mathrm{Mo}(110)$ substrate, decreasing the lattice mismatch and lowering the interfacial energy thanks to the chemical reactions occurring between $\mathrm{NiO}$ and the iron oxide [158]. A similar strategy, employing a $\mathrm{FeO}(111)$ layer grown on $\mathrm{Mo}(110)$, was adopted for the stabilization of $\mathrm{ZnO}(0001)$ films [159].

Metallic buffer layers covering oxide substrates before the deposition of a second metal $\left(\mathrm{M}_{a} / \mathrm{M}_{b} / \mathrm{O}\right)$ can be useful in those cases in which the top metallic layer is a noble metal, employed because of its high stability under environmental conditions. The weak adhesion between the noble metal and the oxide surface often results in the formation of a remarkable number of pinholes (see Section 2.2.2). The insertion of a reactive metal buffer layer between the oxide substrate and the overlayer can enhance the mechanical stability of the $\mathrm{M} / \mathrm{O}$ interface. Comparing the morphology of $\mathrm{Au}$ films grown on the bare $\mathrm{Al}_{2} \mathrm{O}_{3}(0001)$ surface with that obtained on $\mathrm{Co} / \mathrm{Al}_{2} \mathrm{O}_{3}(0001)$, Kamiko et al. observed the formation of highly ordered $\mathrm{Au}(111)$ films on the surface covered by the Co buffer layer, while a disordered film develops on the pristine $\mathrm{Al}_{2} \mathrm{O}_{3}(0001)$ surface [160].

The wetting of the metallic overlayer can be enhanced also by using the $\mathrm{M} / \mathrm{O}_{a} / \mathrm{O}_{b}$ heterostructure. Nozaki et al. found that inserting an $\mathrm{Fe}_{2} \mathrm{O}_{3}$ buffer layer on top of the $\mathrm{MgO}(001)$ surface leads to the development of flat $\mathrm{Fe}$ [161] (Co [162]) films with sub-nanometer thickness, whereas $\mathrm{Fe}(\mathrm{Co})$ films grown on the bare $\mathrm{MgO}$ substrate form a discontinuous layer due to metal islanding. A flat $\mathrm{Fe}(\mathrm{Co})$ morphology allows the establishment of a ferromagnetic long-range order since the early stages of growth, while in the $\mathrm{Fe} / \mathrm{MgO}$ system the ferromagnetism sets in only at higher coverages, when the Fe clusters coalesce and a closed film is formed [163].

\section{Structure and chemistry of ultra-thin oxide films on highly oxidizable substrates}

A large body of literature deals with the preparation and characterization of ultra-thin oxide films on noble and quasi noble metals [3]. These films are often exploited for the study of complex chemical processes involved in heterogeneous catalysis. Phenomena like water interaction with oxide surfaces [164-167] and CO oxidation [168,169] have been deeply investigated thanks to the use of these model systems. A prerequisite to successfully rationalize the complex chemical reactions occurring at oxides surfaces relies on the possibility to obtain highly ordered films, allowing their investigation by means of scanning probe techniques [64,170] and a direct comparison with theoretical models [171]. Comparatively, less progress have been made in the characterization of ultra-thin oxides supported by highly reactive metallic substrates. With the term "highly reactive" we refer to metals for which a stable oxide structure is formed for oxygen exposures performed in UHV conditions (up to $10^{-6}$ mbar).

Such a lack of experimental investigations is partly related to the additional experimental issues encountered when one tries to stabilize an oxide on a highly oxidizable substrates. As a matter of fact, exposure to oxygen can produce severe structural modifications on the substrate, often inducing a roughening of the surface and in turn preventing the development of smooth structures [172]. For this reason, particular care has to be paid for the preparation of well-ordered oxides on such substrates.
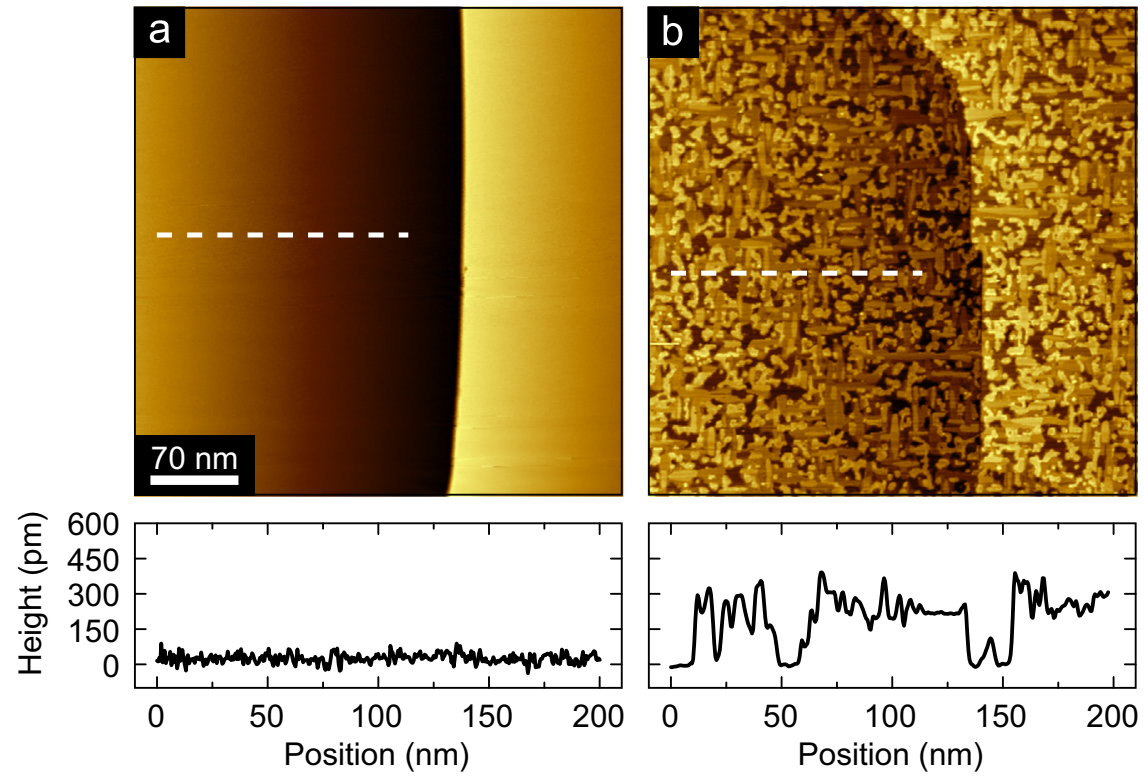

Fig. 9. Oxygen-induced roughening of the $\mathrm{Fe}(001)-p(1 \times 1) \mathrm{O}$ surface. (a) STM topographic image of the $\mathrm{Fe}(001)-p(1 \times 1) \mathrm{O}$ [see Section 4.1]. (b) Morphology obtained after dosing $50 \mathrm{~L}$ of $\mathrm{O}_{2}$ and annealing at $200{ }^{\circ} \mathrm{C}$ in UHV. 
Fig. 9 exemplifies the surface structural modifications induced on the $\mathrm{Fe}(001)-p(1 \times 1) \mathrm{O}$ surface by oxygen exposure (see Section 4.1). While the $\mathrm{Fe}(001)-p(1 \times 1) \mathrm{O}$ substrate is characterized by atomically flat terraces, after dosing $50 \mathrm{~L}$ of molecular oxygen $\mathrm{Fe}$ oxide nuclei develop, increasing considerably the surface roughness. The surface roughening, occurring during the early stages of oxygen dosing, is expected to play an important role in determining the structural quality of the heteroepitaxial ultra-thin oxide film, as indeed observed by Tekiel et al. in their systematic study of the influence of the preparation protocol on the growth of epitaxial $\mathrm{MgO}$ films on $\mathrm{Fe}(001)$ [57]. The $\mathrm{MgO}$ crystal quality depends on the ratio between the $\mathrm{Mg}$ deposition rate $(r)$ and the oxygen partial pressure $(p)$ employed during the reactive deposition. At low $r / p$ values, the overexposure to oxygen causes an excessive substrate oxidation, resulting in a poorly ordered $\mathrm{MgO}$ film, while high $r / p$ values induce the development of understoichiometric $\mathrm{MgO}$ films (see Fig. 10).

\subsection{Late $3 d$ transition metals substrates}

In this section, oxide ultra-thin films stabilized on late $3 d$ transition metals (with the exception of Fe, to which Section 4 is dedicated) are reviewed. Incidentally, such metals can be either antiferromagnetic $(\mathrm{Cr}, \mathrm{Mn})$ or ferromagnetic ( $\mathrm{Fe}, \mathrm{Co}$, $\mathrm{Ni}$, therefore the interaction with an oxide overlayer can have a strong influence on the interfacial magnetic properties, as highlighted in Section 2.4. Because of their high oxygen affinity, ultra-thin layers of native oxides can be stabilized on late $3 d$ magnetic metals by direct exposure to oxygen at relatively low partial pressures. In this case, the crystal quality and morphological order of the oxide overlayer represent a crucial issue, since the oxide crystal structure is generally different with respect to that of the metallic substrate. In

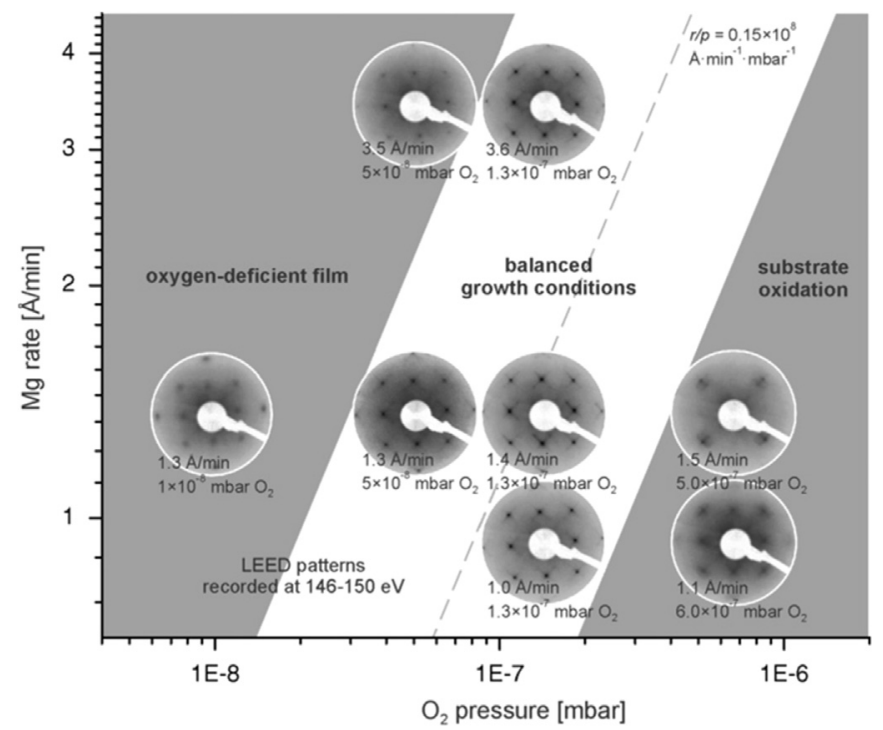

Fig. 10. LEED patterns from $\mathrm{MgO}$ thin films stabilized on $\mathrm{Fe}(001)$ as a function of oxygen partial pressure $(p)$ and $\mathrm{Mg}$ deposition rate $(r)$. The best quality films are obtained for $r / p=0.15 \times 10^{8} \AA \times \min ^{-1} \times \mathrm{mbar}^{-1}$. Reprinted with permission from Ref. [57]. Copyright (2013) by Elsevier. addition, when $3 d$ magnetic metals are employed as substrates for heteroepitaxial growth, particular care has to be paid in the characterization of the interface chemical state, since cationic species from the substrate are likely to be included in the oxide.

Epitaxial $\mathrm{Cr}_{2} \mathrm{O}_{3}$ films have been stabilized on the $\mathrm{Cr}(011)$ surface [173-178]. Oxygen exposure in UHV conditions performed with the substrate held at relatively high temperatures $(603 \mathrm{~K})$ induces the layer-by-layer growth of $\mathrm{Cr}_{2} \mathrm{O}_{3}$, without any interface roughening [174]. On the other hand, oxidation of the $\mathrm{Cr}(001)$ surface produces a polycrystalline oxide film [179].

Oxidation of the $\mathrm{Co}(0001)$ surface results in the stabilization of an ultra-thin $\mathrm{CoO}(111)$ at low oxygen exposure and $\mathrm{Co}_{3} \mathrm{O}_{4}$ at higher exposures [180]. Generally, when $\mathrm{Co}(0001)$ is employed as a substrate for the deposition of heteroepitaxial oxides, the formation of a thin Co oxide interfacial layer is reported. For instance, the $\mathrm{Co}(0001)$ surface has been found to be a suitable substrate for the growth of corundum-structured $\mathrm{Al}_{2} \mathrm{O}_{3}$. In this case the formation of a $\mathrm{CoO}$ thin layer between the $\mathrm{Al}_{2} \mathrm{O}_{3}$ film and the $\mathrm{Co}$ substrate was reported [181]. A $3.5 \AA$ thick Co oxide layer was found also in the interfacial region of ultra-thin chromia films grown on the $\mathrm{Co}(0001)$ surface [182]. Wang et al. studied the $\mathrm{MoO}_{3} / \mathrm{Co}$ layered system, finding a reduction of the oxidation state of Mo in the interfacial region, along with the formation of $\mathrm{Co}$ oxide [183].

The oxidation of $\mathrm{Ni}(001)$ above room temperature produces crystallites with a lateral extension of $50 \AA$ [147], while the formation of a continuous $\mathrm{NiO}(111)$-like layer composed by nanosized domains is observed on Ni(111) [184]. The experimental results in the literature concerning the growth of $\mathrm{Ni}$ supported oxide films generally report a very weak substrate oxidation. $\mathrm{Ni}(001)$ has been used as a template for the growth of $\mathrm{Eu}$ oxide, with a negligible oxidation of the Ni substrate $[185,186]$. $\mathrm{Ni}(111)$ has been used as template for the growth of $\mathrm{Al}_{2} \mathrm{O}_{3}$ films [187,188]. Also in this case, no remarkable oxidation of the $\mathrm{Ni}$ substrate was observed, although Ni-O bonds were detected at the interface. Prevot et al. suggested that the Ni-supported $\mathrm{Al}_{2} \mathrm{O}_{3}$ oxide films can be regarded as a freestanding oxide layers [189]. Ti oxide [190] and V oxide [191] have been stabilized on $\mathrm{Ni}(110)$. In both cases only a weak oxidation of the substrate was reported.

\subsection{Refractory metals}

$4 d(\mathrm{Nb}$ and $\mathrm{Mo})$ and $5 d(\mathrm{Ta}, \mathrm{W}, \mathrm{Re})$ refractory metals are all characterized by a high melting point (above $2273 \mathrm{~K}$ ) and a remarkable hardness. Despite the heat of formation of these oxides is comparable with that of $3 d$ magnetic metals, the onset of bulk oxidation in UHV conditions is observed only at high temperatures or by employing oxidation promoters, such as rare-earths [192,193] or alkali metals [194]. The reluctance of refractory metals towards oxidation relies on the high kinetic constraints experienced by oxygen atoms during their incorporation into the growing oxide film. Nordlander et al. discussed the correlation between the stiffness of the materials 
and the energy barrier for the incorporation of oxygen atoms in subsurface sites. Their findings suggest that the higher the shear modulus, the higher the potential barrier for oxygen penetration through the surface [195]. In this frame, due to their remarkable hardness, refractory metals and their oxidic compounds are expected to display high barriers for oxygen incorporation [196]. Nevertheless, high-quality layers of $\mathrm{WO}_{2}$ were recently stabilized by high temperature oxidation of the $\mathrm{W}(110)$ surface [197]. A structure similar to $\mathrm{WO}_{2} / \mathrm{W}(110)$ is found for the $\mathrm{MoO}_{2}$ oxide layer formed upon high-temperature oxidation of the Mo(110) surface [198].

Thanks to their reluctance towards oxidation, when refractory metals are employed as substrates for the stabilization of heteroepitaxial oxides, the formation of their oxidic phase is seldom reported. The key point for the use of refractory metals substrates is the possibility to explore a large window of temperatures without problems of substrate instability or intermixing between the deposit and the substrate. Mo(001) has been employed as a support for the growth of $\mathrm{MgO}$ [199201] and $\mathrm{CaO}$ thin films [108,109], finding in both cases that the best quality films are obtained after high temperature annealing, at about $1000 \mathrm{~K}$. Either $\mathrm{TiO}_{2}(100)$ or $\mathrm{Ti}_{2} \mathrm{O}_{3}(0001)$ epitaxial layers have been grown on the Mo(110) surface, depending on the film preparation method [202]. Similarly, the $\mathrm{W}(100)$ surface has been reported to be a good support for the growth of $\mathrm{TiO}_{2}(110)$ layers $[203,204]$.

\subsection{Alloys}

Among the class of substrates reactive towards oxidation, the case of binary alloys should be mentioned as well. These are characterized by a crystal structure composed by two different metallic elements, A and B. Generally, alloys are employed since their high melting point allows high-temperature treatments, crucial to obtain ordered crystalline structures. The preferred route for obtaining an epitaxial oxide over a binary alloy substrate is typically represented by the direct oxidation of the surface. If the atomic species B possesses a larger oxygen affinity with respect to $\mathrm{A}$, the oxidation will primarily induce the segregation and oxidation of $\mathrm{B}$ atoms, producing a $\mathrm{BO}_{x}$ layer covering the surface [205-207]. In this case the alloy should release the reactive metal slowly, so that the oxidation process takes place at a moderate rate, which constitutes a crucial prerequisite for the formation of good-quality crystalline oxide films. As an example, $\mathrm{Pt}_{3} \mathrm{Zr}(0001)$ [208] and $\mathrm{Pd}_{3} \mathrm{Zr}(0001)$ [209] substrates have been exploited to grow well-ordered $\mathrm{ZrO}_{2}$ films by means of post oxidation.

The paradigmatic example of an oxide supported by an alloy substrate is represented by the binary compound $\mathrm{NiAl}$, employed as a substrate for the stabilization of well-ordered $\mathrm{Al}_{2} \mathrm{O}_{3}$ films. While direct oxidation of $\mathrm{Al}$ surfaces produces amorphous layers [210,211], high-temperature oxidation of either $\mathrm{NiAl}(110)$ [212,213] or $\mathrm{Ni}_{3} \mathrm{Al}(111)$ [214] induces the formation of high-crystal-quality $\mathrm{Al}_{2} \mathrm{O}_{3}$ films. $\mathrm{Al}_{2} \mathrm{O}_{3}$ has been stabilized also by oxidation of $\mathrm{Cu}-9$ at\% $\mathrm{Al}(111)$ [215].

Surface-confined alloys, obtained by deposition of metallic films on metallic substrates, have also been used as a template for the stabilization of ultra-thin oxide films. The oxidation of a $\mathrm{Ti}-\mathrm{Au}$ alloy formed at the $\mathrm{Au}(111)$ surface induces the development of $\mathrm{TiO}_{2}$ nanocrystals [216], while Co oxide develops from the oxidation of a surface-confined Co-Pt alloy grown on $\mathrm{Pt}(111)$ [217].

\section{Ultra-thin oxides on $\mathrm{Fe}(001)$}

\section{1. $\mathrm{Fe}(001)$ and $\mathrm{Fe}(001)-p(1 \times 1) O$ substrates}

$\mathrm{Fe}(001)$ substrates are available in the form of whiskers [218-220] or bulk single crystals [220]. In addition, high quality $\mathrm{Fe}(001)$ surfaces can be obtained by depositing thick $\mathrm{Fe}$ films (thickness above $100 \mathrm{~nm}$ ) onto a polished $\mathrm{MgO}$ substrate by means of MBE [221]. Such Fe(001) films display the same properties of bulk iron, but compared to $\mathrm{Fe}(001)$ single crystals are cheaper and much easier to clean from contaminants.

The $\mathrm{Fe}(001)-p(1 \times 1) \mathrm{O}$ surface, characterized by a single layer of oxygen atoms adsorbed on the fourfold hollow sites of $\mathrm{Fe}(001)$, can be obtained by dosing molecular oxygen onto the clean $\mathrm{Fe}(001)$ surface and annealing the sample at high temperatures (above $700 \mathrm{~K}$ ). Although several preparation methods can be found in the literature, mainly differing in the oxygen amount employed to saturate the clean surface (ranging from 0.6 L [222] to $30 \mathrm{~L}$ [223]), according to Lu et al. the final heating step annihilates any surface disorder induced by oxygen overdosing, leaving just one well-ordered layer of oxygen atoms [224].

Fig. 11 displays the effects induced by the oxidation process and high-temperature annealing on the $\mathrm{Fe}(001)$ mesoscopic morphology [223]. The oxygen-free $\mathrm{Fe}(001)$ sample is characterized by relatively small terraces (average width equal to about $30 \mathrm{~nm}$ ) separated by monoatomic steps (with a topographic height of $143 \mathrm{pm}$ ). Screw dislocations arising from strain relaxation are visible on the surface. Oxygen adsorption and subsequent annealing induce a noticeable mass rearrangement over the whole surface, and the $\mathrm{Fe}(001)-p(1 \times 1) \mathrm{O}$ reconstruction displays wide atomically flat terraces (average width equal to about $140 \mathrm{~nm}$ ), separated by multilayer steps. The product between the step density and the mean step height before and after oxidation is the same, indicating that the mesoscopic structure of the $\mathrm{Fe}(001)-p(1 \times 1) \mathrm{O}$ surface results from the bunching of the steps present on the pristine $\mathrm{Fe}(001)$ surface.

At the atomic level, oxygen atoms arrange in a highly ordered overlayer, in registry with the substrate [225-227]. The oxygeninduced enhancement of the atomic corrugation allows the achievement of atomic resolution on the $\mathrm{Fe}(001)-p(1 \times 1) \mathrm{O}$ substrate, difficult to obtain on the clean $\mathrm{Fe}(001)$ surface $[228,229]$. The atomically resolved STM image acquired on the $\mathrm{Fe}(001)-p(1 \times 1) \mathrm{O}$, displayed in Fig. 12 , shows that large defectfree regions are present on this surface. The oxygen atoms reside in the fourfold symmetric surface hollow sites, protruding slightly above the $\mathrm{Fe}(001)$ topmost layer. Since the oxygen overlayer is not coplanar with the $\mathrm{Fe}(001)$ surface layer, an electric dipole directed normal to the surface appears. As a consequence, the surface work 

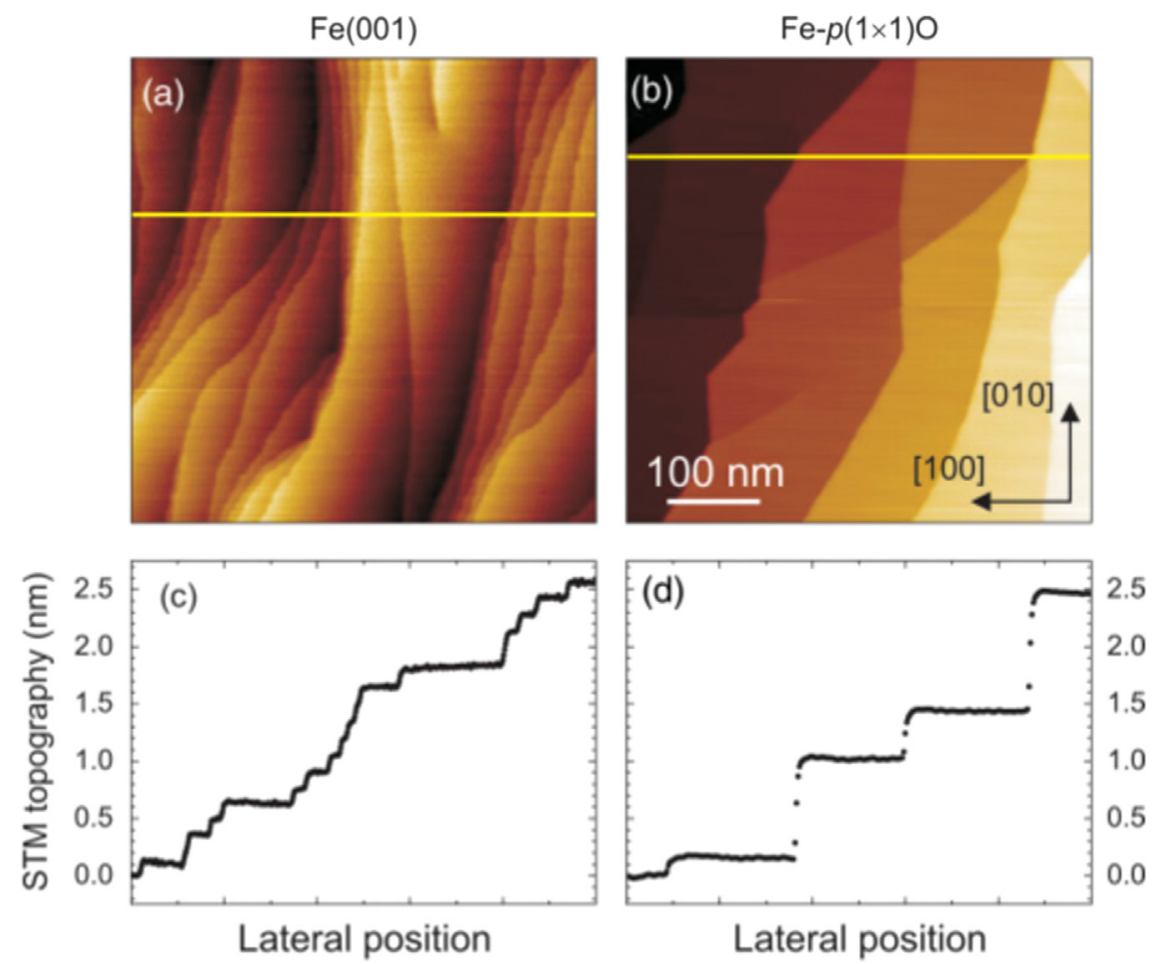

Fig. 11. (a and b) Comparison between the mesoscopic morphology of $\mathrm{Fe}(001)$ and $\mathrm{Fe}(001)-p(1 \times 1) \mathrm{O}$ (image size is $500 \times 500 \mathrm{~nm}^{2}$ ). (c and d) Profiles measured along the scan lines drawn in panels (a) and (b), respectively. Reprinted with permission from Ref. [223]. Copyright (2011) by the American Physical Society.

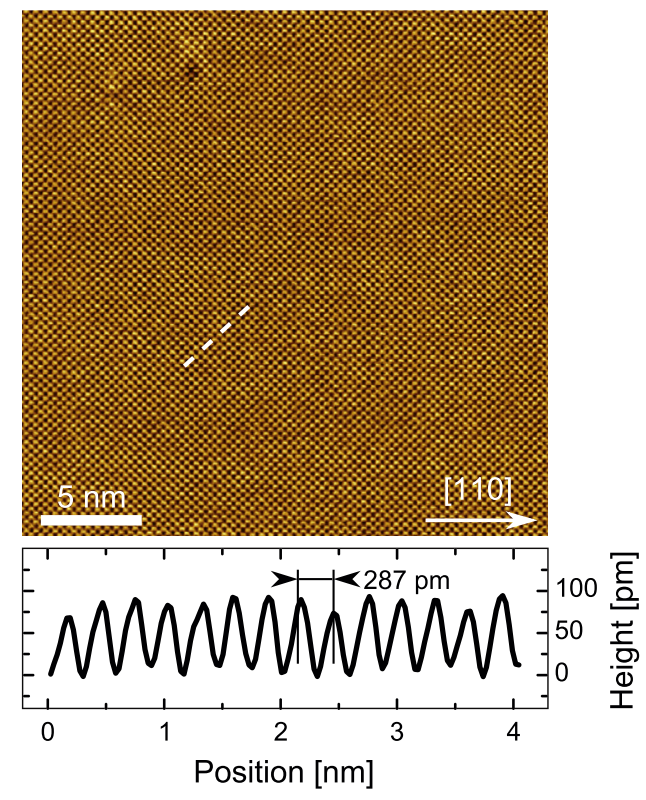

Fig. 12. Atomically resolved STM image illustrating the high degree of order at the $\mathrm{Fe}(001)-p(1 \times 1) \mathrm{O}$ surface.

function of the oxidized sample is about $0.6-0.7 \mathrm{eV}$ higher than that of the oxygen-free surface [230,231]. The oxygen overlayer also induces an outward relaxation of the topmost Fe layer, with the first Fe-Fe interlayer spacing increased with respect to the bulk value. First principles calculations performed by Chubb et al. show a 23\% increase [232], while Legg et al. inferred an expansion of 7\% from LEED data [233]. More recently, Parhiar et al. determined an increase of $16 \%$ by combining x-ray diffraction and $a b$ initio calculations [234]. The oxygen-induced relaxation of the topmost layers has been found to enhance the surface spindependent effects with respect to oxygen-free $\mathrm{Fe}(001)$ [235]. Moreover, the surface magnetic moment oscillates during $\mathrm{Fe}$ homoepitaxial growth on the $\mathrm{Fe}(001)-p(1 \times 1) \mathrm{O}$ [236,237], a phenomenon recently related to the different oxygen adsorption geometry on the islands and on the atomically flat terraces [238].

The first layer of oxygen atoms adsorbed on the $\mathrm{Fe}(001)$ hollow sites is often described as a chemisorbed phase, preceding the onset of bulk oxidation. However, the atomic structure of the $\mathrm{Fe}(001)-p(1 \times 1) \mathrm{O}$ surface has been suggested to be similar to an ultra-thin rocksalt-type $\mathrm{FeO}$ film accommodated on the $\mathrm{Fe}(001)$ surface, as evidenced by the theoretical investigation of Blonski et al., where the presence of strong covalent $\mathrm{Fe}-\mathrm{O}$ bonds formed by $\mathrm{Fe}-3 d-\mathrm{O}-2 p$ hybrid orbitals, similar to those of bulk $\mathrm{FeO}$, was highlighted [231]. These theoretical results are experimentally confirmed by low electron-kinetic-energy AES spectroscopy [see Fig. 13(a)], which is particularly sensitive to the local chemical environment of surface atoms. While the spectrum of the oxygen-free surface is characterized by a single peak located at about $47 \mathrm{eV}$, corresponding to the MVV transition of clean $\mathrm{Fe}(001)$, upon oxygen adsorption a small shoulder appears at about $38 \mathrm{eV}$. This feature is more evident in the spectra acquired by tilting the sample with respect to the emitted electron beam, indicating that such a feature is related to the surface electronic structure. The same feature is found also on Fe oxide surfaces produced by oxidation of either bulk Fe samples [239-241] or $\mathrm{W}(110)$-supported $\mathrm{Fe}$ monolayer films exposed to oxygen 

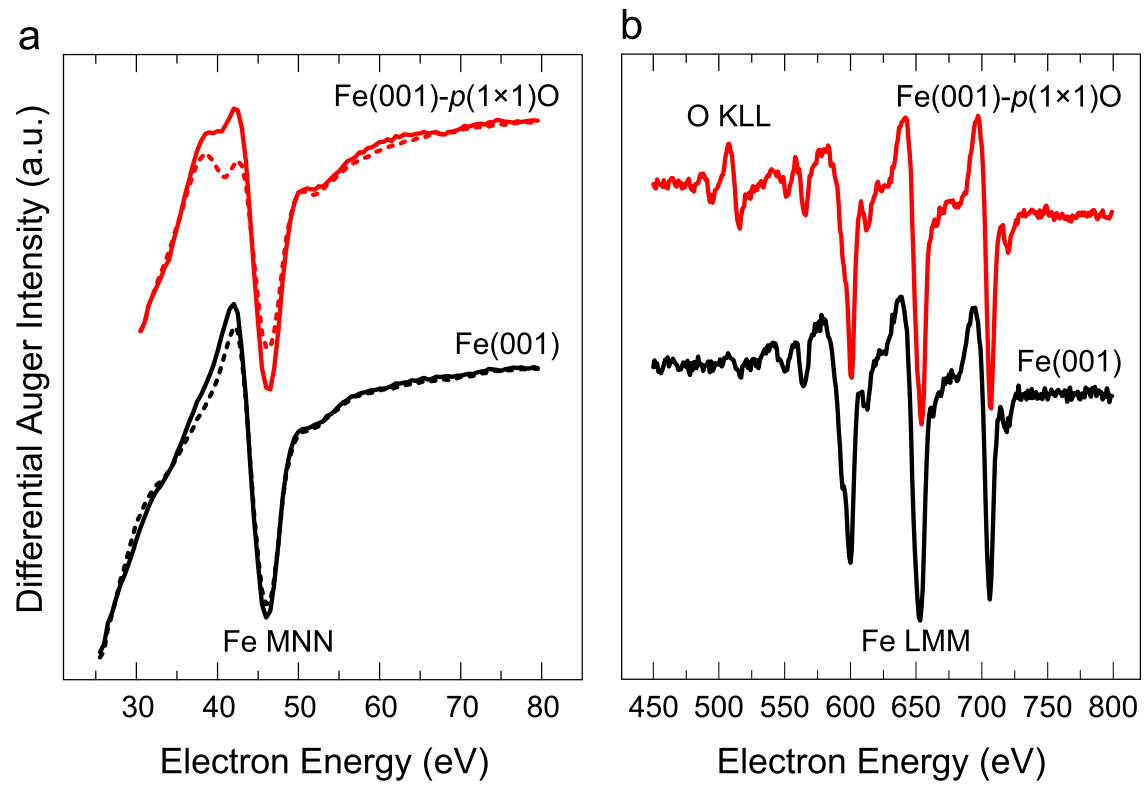

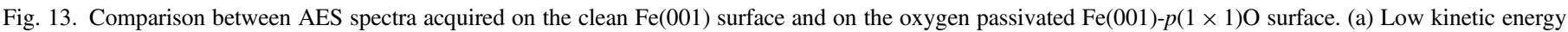

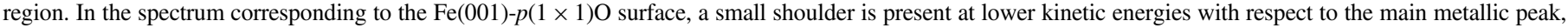

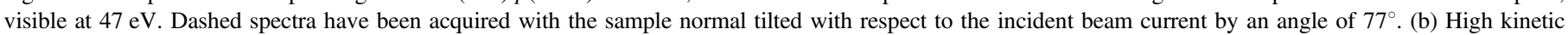
energy region. Upon oxygen adsorption, the peaks corresponding to oxygen KLL transitions arise.

[242], indicating that the chemical bonds between $\mathrm{Fe}$ and $\mathrm{O}$ on the $\mathrm{Fe}(001)-p(1 \times 1) \mathrm{O}$ surface are very similar to those found on the surfaces of Fe oxides. Fig. 13(b) displays the spectra acquired on the $\mathrm{Fe}(001)$ and $\mathrm{Fe}(001)-p(1 \times 1) \mathrm{O}$ samples in the high kinetic energy region. Upon oxygen adsorption, the peaks related to the O KLL transitions arise, while the Fe LMM transitions are not significantly perturbed by the oxygen overlayer.

STS acquired on the $\mathrm{Fe}(001)-p(1 \times 1) \mathrm{O}$ surface confirms that substantial modifications of the $\mathrm{Fe}(001)$ electronic structure are induced by the ordered oxygen overlayer. The clean $\mathrm{Fe}(001)$ surface is characterized by an intense surface state at $0.17 \mathrm{eV}$ above $E_{\mathrm{F}}$ [243], weakly shifted by $0.04 \mathrm{eV}$ to higher energies when isolated oxygen impurities are present on the surface [244]. On the other hand, in $d I / d V$ STS data acquired on the $\mathrm{Fe}(001)-p(1 \times 1) \mathrm{O}$ surface, displayed in the inset of Fig. 14, two peaks are located at about $1 \mathrm{eV}$ above and $0.5 \mathrm{eV}$ below $E_{\mathrm{F}}$, respectively [226]. These spectroscopic features are better resolved in the spectrum reported in the main panel of Fig. 14, obtained by normalizing the STS curves to the tunneling barrier transmission coefficient [226,245,246].

A direct comparison between the measured and the computed electronic structure for the $\mathrm{Fe}(001)-p(1 \times 1) \mathrm{O}$ surface is obtained by considering the calculated density of states integrated over a proper region of the first surface Brillouin zone [226]. Fig. 15 displays the computed density of states, evaluated in the two surface layers (Fe and $\mathrm{O}$ ). The majority and minority components are reported in the upper and lower panels, respectively, while the dotted line corresponds to the total DOS. The minority-spin state located at $0.17 \mathrm{eV}$, characteristic of oxygen-free $\mathrm{Fe}(001)$ [243], is not present on the $\mathrm{Fe}(001)-p(1 \times 1) \mathrm{O}$ surface, while a feature at $0.8 \mathrm{eV}$ is detected. This is attributed to a surface resonance, because of its large amplitude in the vacuum region. This unoccupied minority surface state is in very good agreement with the measured position of the large peak at positive bias in STS data. A better agreement between experiment and theory can be obtained by considering that, because of the faster decay towards vacuum of the wave functions at large $k$ values, the STS probing method possesses a higher sensitivity to states around the $\Gamma$ point. By assuming that only $1 / 5$ of the first surface Brillouin zone around $\Gamma$ contributes to the STS signal [Fig. 15(b)], one finds that features at about $0.35 \mathrm{eV}$ below $E_{\mathrm{F}}$ appear in the partial density of minority spin states, which can be readily associated to the peak detected at about $-0.5 \mathrm{eV}$ in the STS spectra.

In conclusion, the available experimental and theoretical data suggest to consider the $\mathrm{Fe}(001)-p(1 \times 1) \mathrm{O}$ as a completely new phase with respect to oxygen-free $\mathrm{Fe}(001)$, characterized by its own structural, electronic and magnetic properties.

\subsection{Wetting layer oxides on $\mathrm{Fe}(001)$}

A suitable strategy to obtain a sharp interface between a single-layer of oxide and a reactive substrate could be to exploit the oxygen adsorbed on the surface before metal deposition since, in this case, the amount of oxygen available is well defined. Preloaded oxygen on surface and subsurface sites has been employed for the preparation of twodimensional films of vanadium, niobium, and molybdenum oxides on $\mathrm{Cu}_{3} \mathrm{Au}(100)$ [247]. An incomplete $\mathrm{BaO}_{x}$ layer was obtained by depositing $\mathrm{Ba}$ atoms on the $\mathrm{O}(2 \times 1) \mathrm{Ni}(110)$ surface [248]. More recently, a sharp interface between a $\mathrm{Ni}$ thin film and a single layer of $\mathrm{NiO}$ was obtained by depositing $\mathrm{Ni}$ onto a pre-oxidized $\mathrm{Cu}(100)$ surface [249], and even a ternary $\mathrm{Ba}_{x} \mathrm{Ti}_{2} \mathrm{O}_{3}$ oxide has been obtained by depositing $\mathrm{Ba}$ 


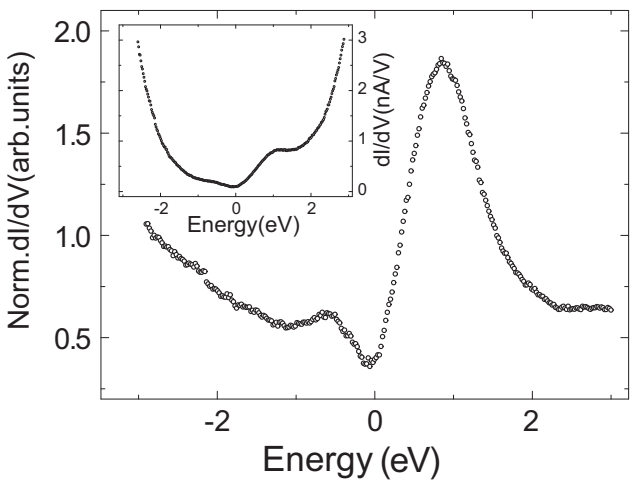

Fig. 14. Conductance spectra, normalized to the tunneling barrier transmission coefficient, acquired on the $\mathrm{Fe}(001)-p(1 \times 1) \mathrm{O}$ surface. The inset displays the raw data. Reprinted with permission from Ref. [226]. Copyright (2009) by the American Physical Society.
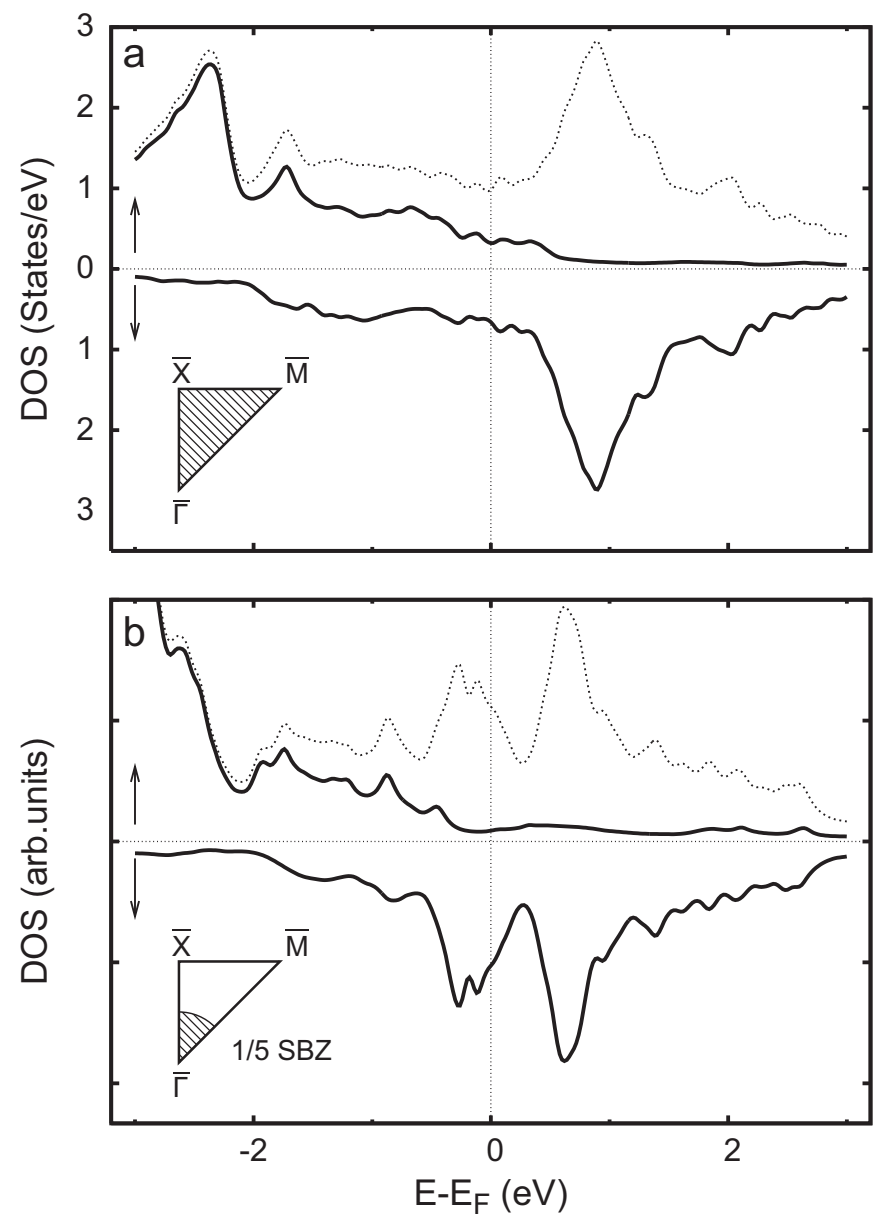

Fig. 15. (a) Computed density of $\mathrm{Fe}(001)-p(1 \times 1) \mathrm{O}$ states in the surface region (surface and subsurface layer), integrated over the entire first surface Brillouin zone. (b) Computed density of $\mathrm{Fe}(001)-p(1 \times 1) \mathrm{O}$ states integrated only over $1 / 5$ of the surface Brillouin zone around $\Gamma$. Up arrow: majority-spin component; down arrow: minority-spin component; dotted line: total density of states. Reprinted with permission from Ref. [226]. Copyright (2009) by the American Physical Society.

atoms onto an ultra-thin $\mathrm{Ti}_{2} \mathrm{O}_{3}$ film supported on $\mathrm{Au}(111)$ [250]. The natural starting point for the growth of $\mathrm{Fe}(001)$ supported ultra-thin oxides is the $\mathrm{Fe}(001)-p(1 \times 1) \mathrm{O}$ surface. A
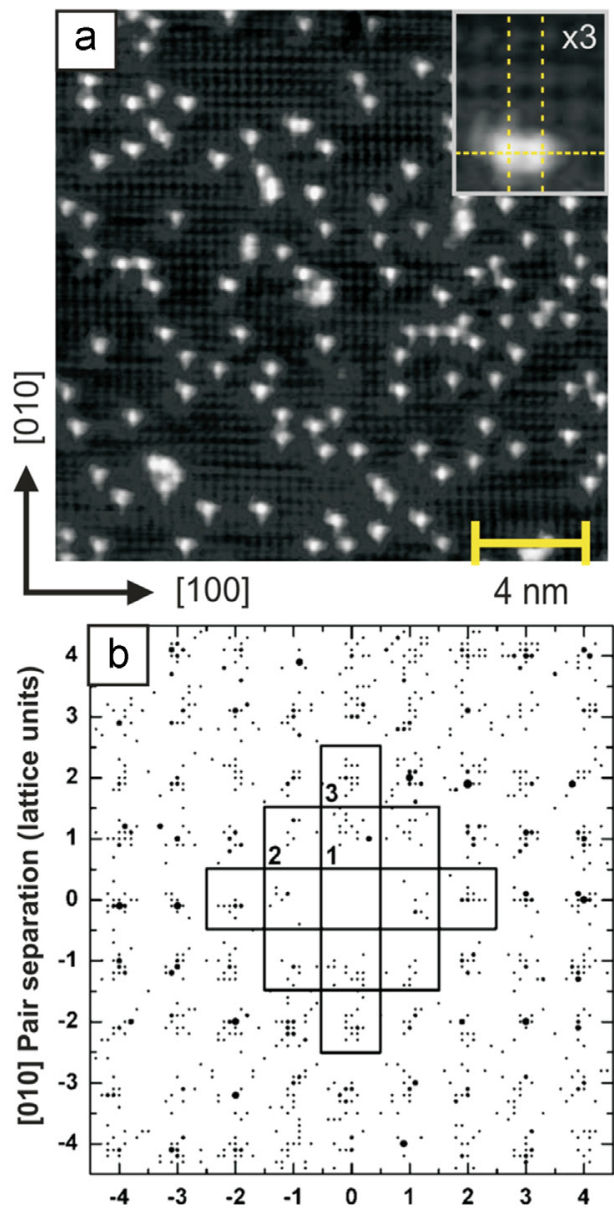

[100] Pair separation (lattice units)

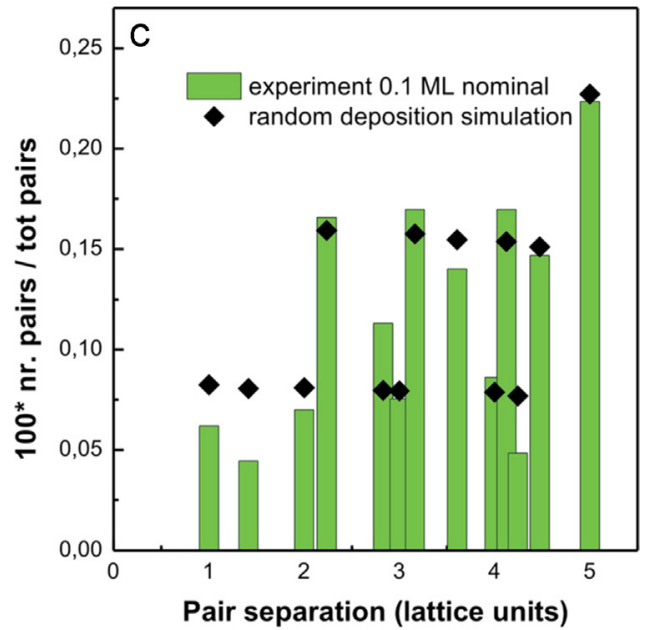

Fig. 16. (a) Atomically resolved STM image acquired after deposition of $0.1 \mathrm{ML} \mathrm{Cr}$ on the $\mathrm{Fe}(001)-p(1 \times 1) \mathrm{O}$ substrate kept at high temperature (673 K). The inset shows a blow-up of a $\mathrm{Cr}$ first nearest-neighbor pair, where the alignment with the substrate lattice is indicated by the dashed lines. (b) Statistical distribution of relative $\mathrm{Cr}$ pair co-ordinates of the adatoms observed in panel (a). Regions corresponding to the first, second, and third nearest-neighbor separations are evidenced in the figure. (c) Comparison between the experimental results reported in panel (b), integrated over the possible directions (vertical bars), and a simulated random distribution (symbols). Reprinted with permission from Ref. [252]. Copyright (2013) by AIP Publishing LLC. 

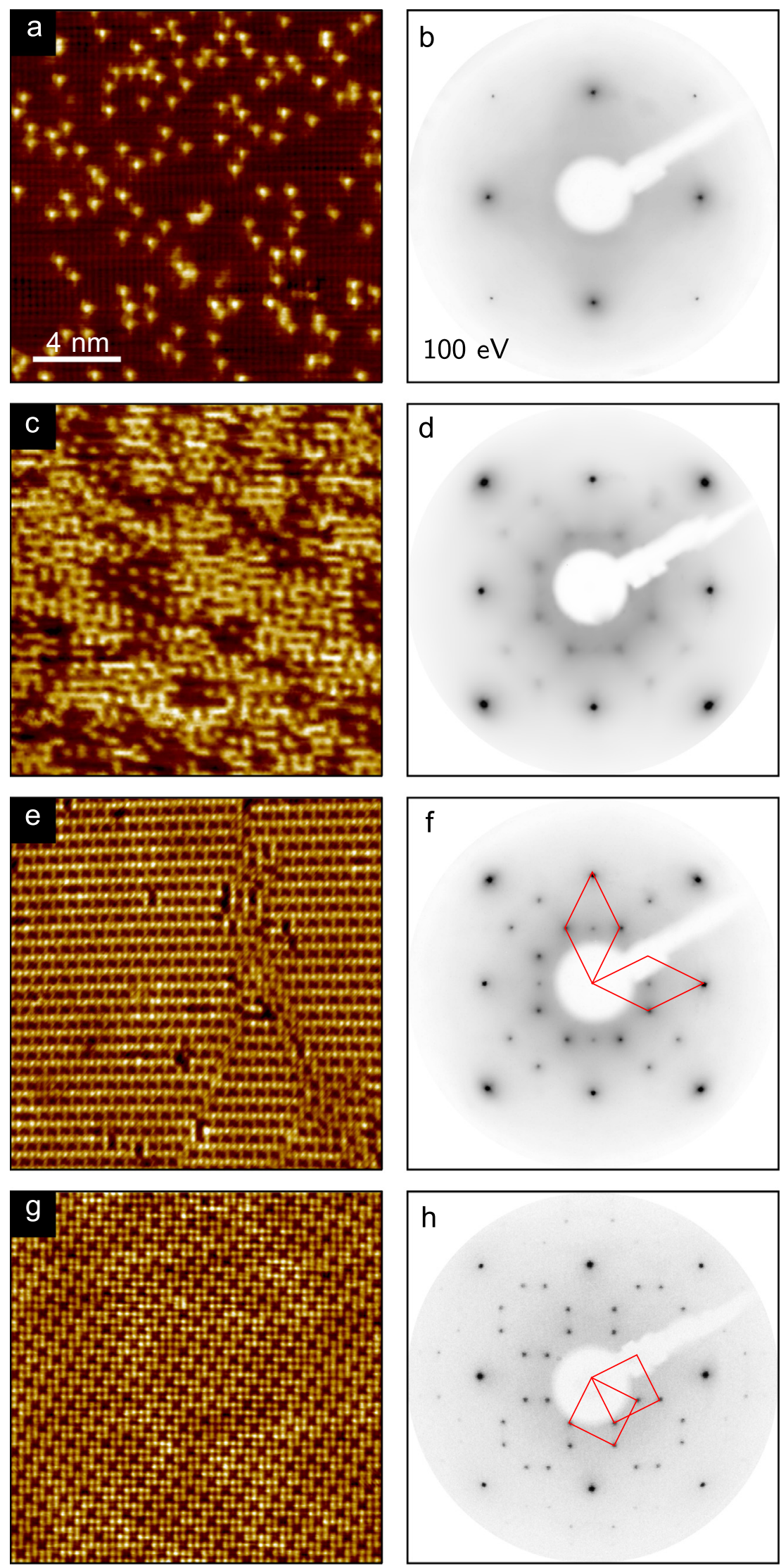

Fig. 17. High-resolution STM images acquired after deposition of (a) 0.1 , (c) 0.4 , (e) 0.75 , and (g) $0.8 \mathrm{ML}$ of $\mathrm{Cr}$ on $\mathrm{Fe}(001)-p(1 \times 1) \mathrm{O}$, respectively ( $I=5 \mathrm{nA}$, $V=100 \mathrm{mV}$ ). In the right column the corresponding LEED patterns (electron beam energy $E=100 \mathrm{eV}$ ) are reported. The reciprocal unit cells of the two rotational domains are superimposed on panels (f) and (h). Adapted with permission from Ref. [251]. Copyright (2013) by the American Physical Society. 

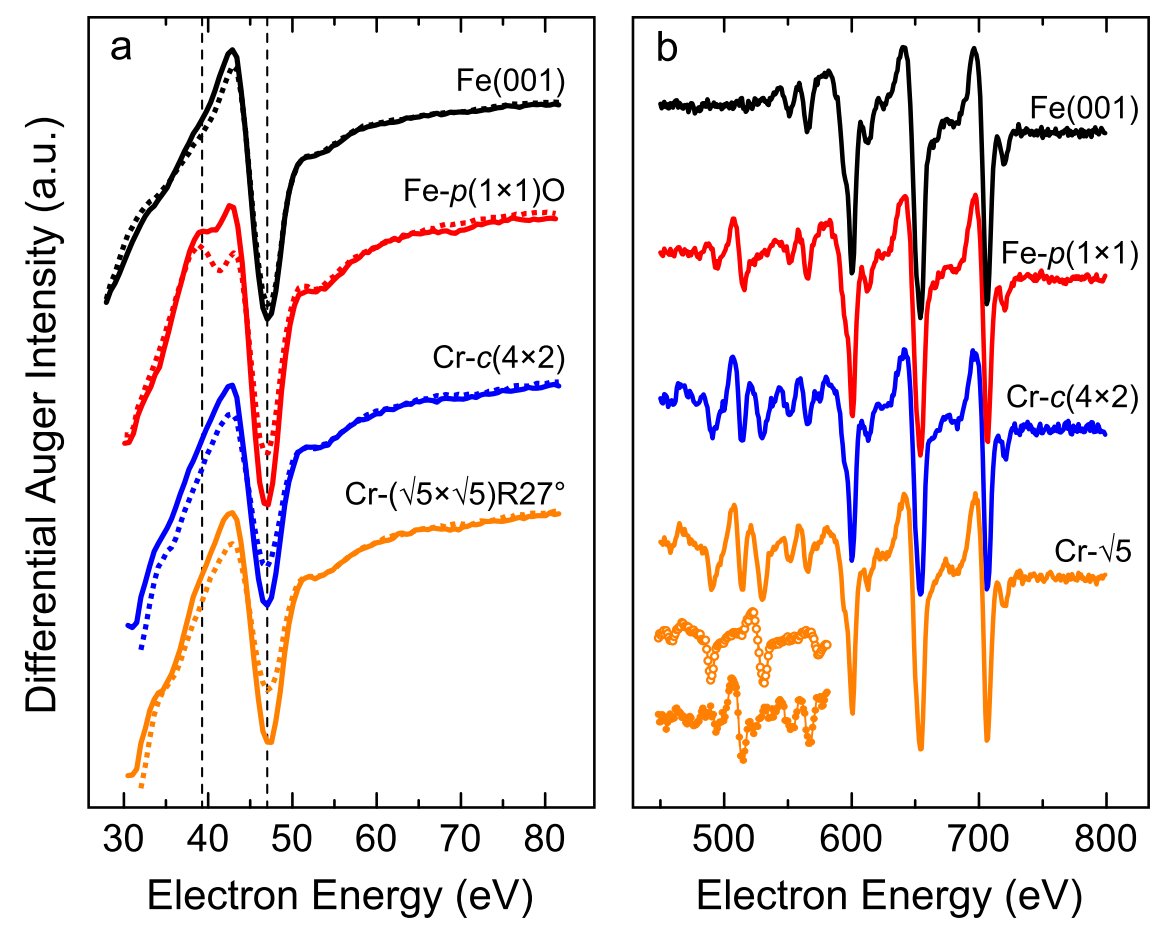

Fig. 18. (a) Variation of the Fe MNN AES lineshape at normal (continuous line) and grazing incidence (dotted line, sample normal $77^{\circ}$ tilted with respect to the emitted beam) for the different $\mathrm{Cr}_{x} \mathrm{O}_{y}$ phases observed for ultra-thin $\mathrm{Cr} / \mathrm{Fe}(001)-p(1 \times 1) \mathrm{O}$ films. The vertical dashed lines indicate the maximum of the feature related to Fe-O bonds $(39.5 \mathrm{eV}$ ) and the minimum of the metallic Fe peak $(47 \mathrm{eV})$. (b) Corresponding high kinetic energy region for the same samples in (a). The bottom-left part reports the spectrum of a reference Cr sample (open symbols) and the result (full symbols) obtained by subtracting the former from the AES spectrum observed on the $(\sqrt{5} \times \sqrt{5}) R 27^{\circ}$ sample. Panel (a) adapted from Ref. [251]. Copyright (2013) by the American Physical Society.
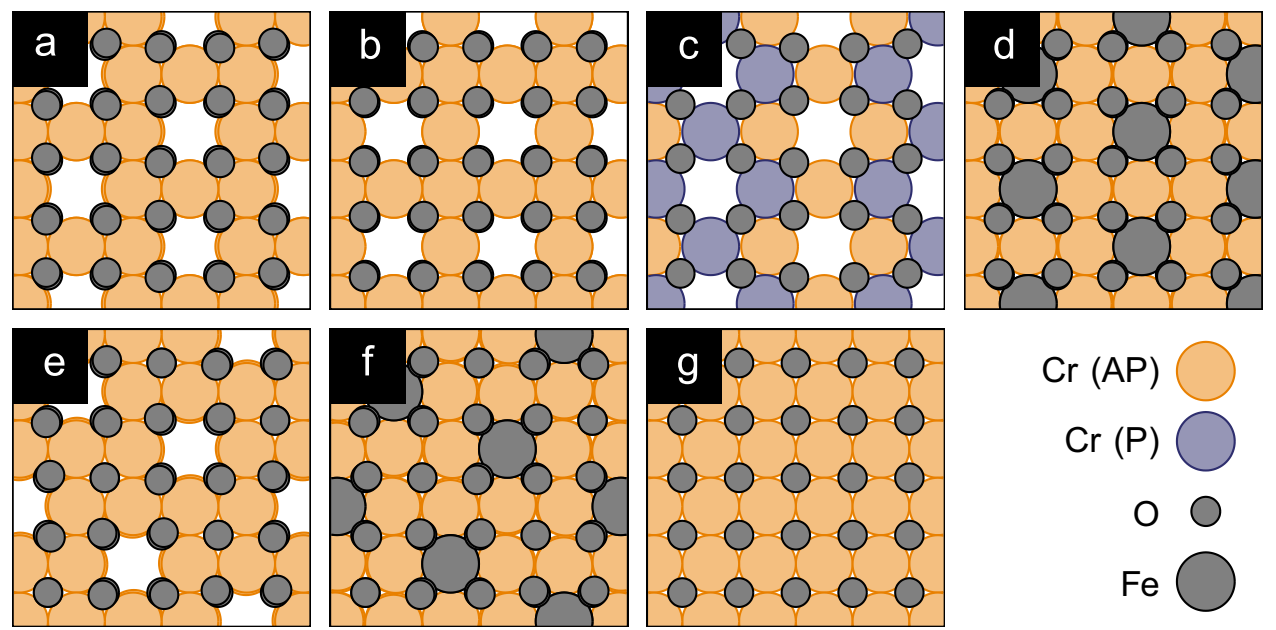

Fig. 19. Top view of structures simulated by DFT: (a) $c(4 \times 2)$ phase with metal vacancies, (b) $p(2 \times 2)$, (c) $p(4 \times 2)$, (d) $c(4 \times 2)$ with Fe atoms filling the vacancies, (e) $(\sqrt{5} \times \sqrt{5}) R 27^{\circ}$ with metal vacancies, (f) $(\sqrt{5} \times \sqrt{5}) R 27^{\circ}$ with Fe atoms filling the vacancies, and $(\mathrm{g}) p(1 \times 1)$. Only atoms above the Fe( $(001)$ surface layer are depicted. $\mathrm{Cr}(\mathrm{AP}) /(\mathrm{P})$ refers to atoms with magnetic moment antiparallel/parallel to the one of the Fe surfaces. Reprinted with permission from Ref. [251]. Copyright (2013) by the American Physical Society.

noteworthy case in which this strategy has been successful is that of Cr oxide single layers [251,252].

Fig. 16(a) focuses on the surface morphology of $0.1 \mathrm{ML} \mathrm{Cr}$ grown on the $\mathrm{Fe}(001)-p(1 \times 1) \mathrm{O}$ surface, with the substrate kept at $673 \mathrm{~K}$ during $\mathrm{Cr}$ deposition. Isolated atomic-scale features, imaged as protrusions in the constant current STM image, are visible on the substrate. The observation of isolated atoms in STM measurements acquired at room temperature, where adatoms are usually highly mobile on uniform surfaces, suggests that deposited $\mathrm{Cr}$ atoms are embedded into the topmost substrate layer. Fig. 16(b) and (c) displays the outcome of a statistical analysis performed on the atomically resolved STM images, from which it is possible to notice that the $\mathrm{Cr}$ pair distribution does not significantly deviate from a random distribution. In a similar analysis performed for $\mathrm{Cr}$ deposition on oxygen-free $\mathrm{Fe}(001)$ [253], no nearest neighbor $\mathrm{Cr}$ adatoms were found, indicating an effective repulsion between $\mathrm{Cr}$ atoms, which has been attributed to the presence 
Table 5

Summary of DFT energetics. The unit cell of each structure covers $N_{1 \times 1}$ primitive unit cells of $\mathrm{Fe}(001)$ and includes $N_{\mathrm{Cr}}\left(N_{\mathrm{Fe}}\right) \mathrm{Cr}(\mathrm{Fe})$ atoms. $F$ indicates the formation energy per $\mathrm{Cr}$ atom. Refer to Fig. 19 for structural models. Reprinted with permission from Ref. [251]. Copyright (2013) by the American Physical Society.

\begin{tabular}{lllllll}
\hline Coverage & Structure & Periodicity & $N_{1 \times 1}$ & $N_{\mathrm{Cr}}$ & $N_{\mathrm{Fe}}$ & $F(\mathrm{eV})$ \\
\hline $0.75 \mathrm{ML}$ & (a) & $c(4 \times 2)$ & 4 & 3 & 0 & -1.12 \\
& (b) & $p(2 \times 2)$ & 4 & 3 & 0 & -1.05 \\
& $(\mathrm{c})^{\mathrm{a}}$ & $p(4 \times 2)$ & 8 & 6 & 0 & -0.99 \\
& $(\mathrm{~d})^{\mathrm{b}}$ & $c(4 \times 2)$ & 4 & 3 & 1 & -0.70 \\
$0.80 \mathrm{ML}$ & $(\mathrm{e})$ & $(\sqrt{5} \times \sqrt{5}) R 27^{\circ}$ & 5 & 4 & 0 & -1.02 \\
& $(\mathrm{f})^{\mathrm{b}}$ & $(\sqrt{5} \times \sqrt{5}) R 27^{\circ}$ & 5 & 4 & 1 & -0.69 \\
$1.00 \mathrm{ML}$ & $(\mathrm{g})$ & $p(1 \times 1)$ & 1 & 1 & 0 & -0.55
\end{tabular}

${ }^{\mathrm{a}}$ Antiferromagnetic coupling within the $\mathrm{Cr}$ layer.

${ }^{\mathrm{b}}$ Vacancies filled by Fe atoms.
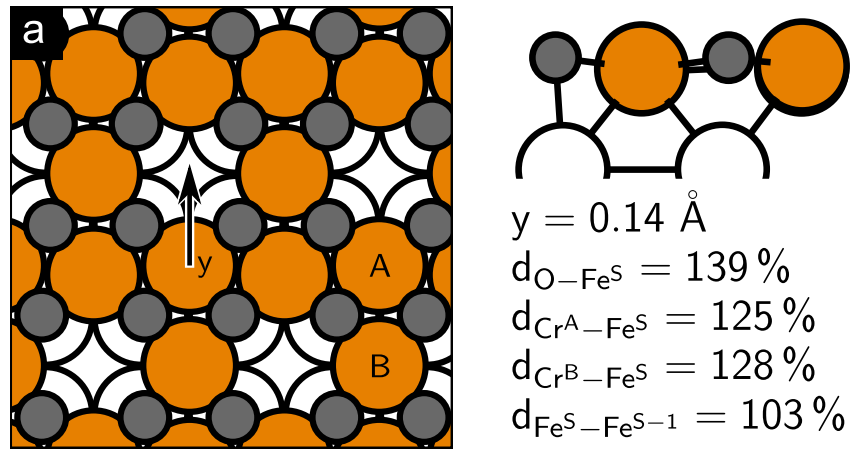

$\mathrm{y}=0.14 \AA$

$\mathrm{d}_{\mathrm{O}-\mathrm{Fe}_{\mathrm{e}} \mathrm{s}}=139 \%$

$\mathrm{d}_{\mathrm{Cr}^{\mathrm{A}}-\mathrm{Fe}^{\mathrm{s}}}=125 \%$

$\mathrm{d}_{\mathrm{Cr}^{\mathrm{B}}-\mathrm{Fe}^{\mathrm{S}}}=128 \%$

$\mathrm{d}_{\mathrm{Fe}^{\mathrm{S}}-\mathrm{Fe}^{\mathrm{S}-1}=103 \%}$
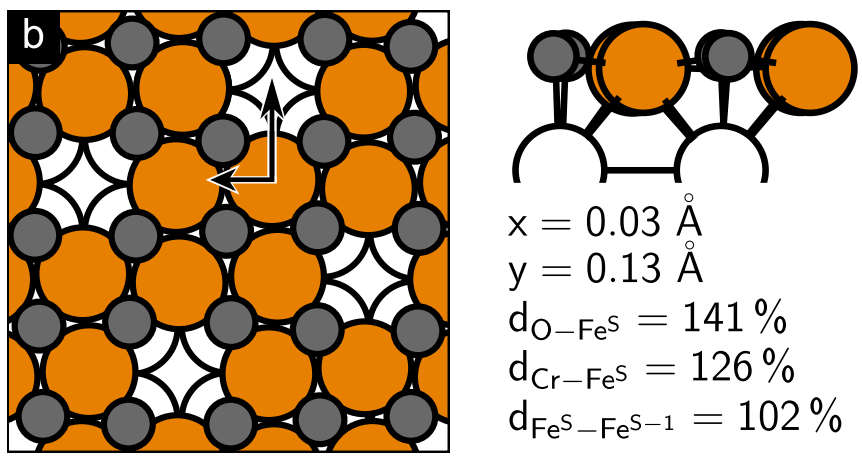

$x=0.03 \AA$

$y=0.13 \AA$

$\mathrm{d}_{\mathrm{O}-\mathrm{Fe}_{\mathrm{e}}^{\mathrm{s}}}=141 \%$

$\mathrm{d}_{\mathrm{Cr}-\mathrm{Fe}^{\mathrm{S}}}=126 \%$

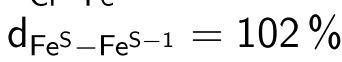

Fig. 20. Top and side views of the (a) $c(4 \times 2)$ and (b) $(\sqrt{5} \times \sqrt{5}) R 27$ superstructures. The arrows indicate the in-plane displacements $(x, y)$ of the $\mathrm{Cr}$ atoms. Interlayer spacings $d$ are given in percentages of the one in bulk iron. By $\mathrm{Fe}^{\mathrm{S}}$ and $\mathrm{Fe}^{\mathrm{S}-1}$ we indicate iron atoms in the first and the second layer, respectively. Reprinted with permission from Ref. [251]. Copyright (2013) by the American Physical Society.

of magnetic frustration [254]. This repulsive interaction between $\mathrm{Cr}$ atoms embedded in the $\mathrm{Fe}(001)$ topmost layer seems to be suppressed by the oxygen overlayer present on the $\mathrm{Fe}(001)-p(1 \times 1) \mathrm{O}$ substrate.

For higher coverages the $\mathrm{Cr}$ atoms self-organize in highly ordered structures, as evidenced in Fig. 17. In Fig. 17(c), corresponding to a $\mathrm{Cr}$ coverage of $0.4 \mathrm{ML}$, the regions in which the $\mathrm{Cr}$ density is higher, appear to be locally ordered, as
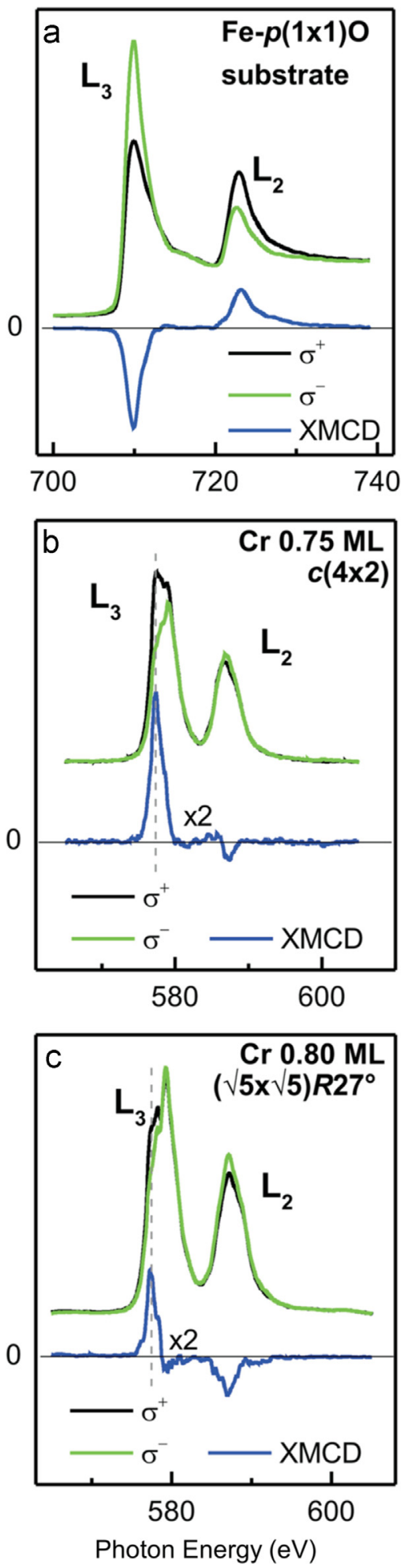

Fig. 21. XAS spectra at $\mathrm{L}_{2,3}$ edges taken with either positive (black lines) or negative (green lines) photon helicity and XMCD spectra (blue lines) of panel (a) $\mathrm{Fe}(001)-p(1 \times 1) \mathrm{O}$ substrate; panel (b) $c(4 \times 2)$ phase; and panel (c) $(\sqrt{5} \times \sqrt{5}) R 27^{\circ}$ phase. The dashed vertical lines indicate the position of the XMCD main peak. Adapted with permission from Ref. [252]. Copyright (2013) by AIP Publishing LLC. (For interpretation of the references to color in this figure caption, the reader is referred to the web version of this paper.) 

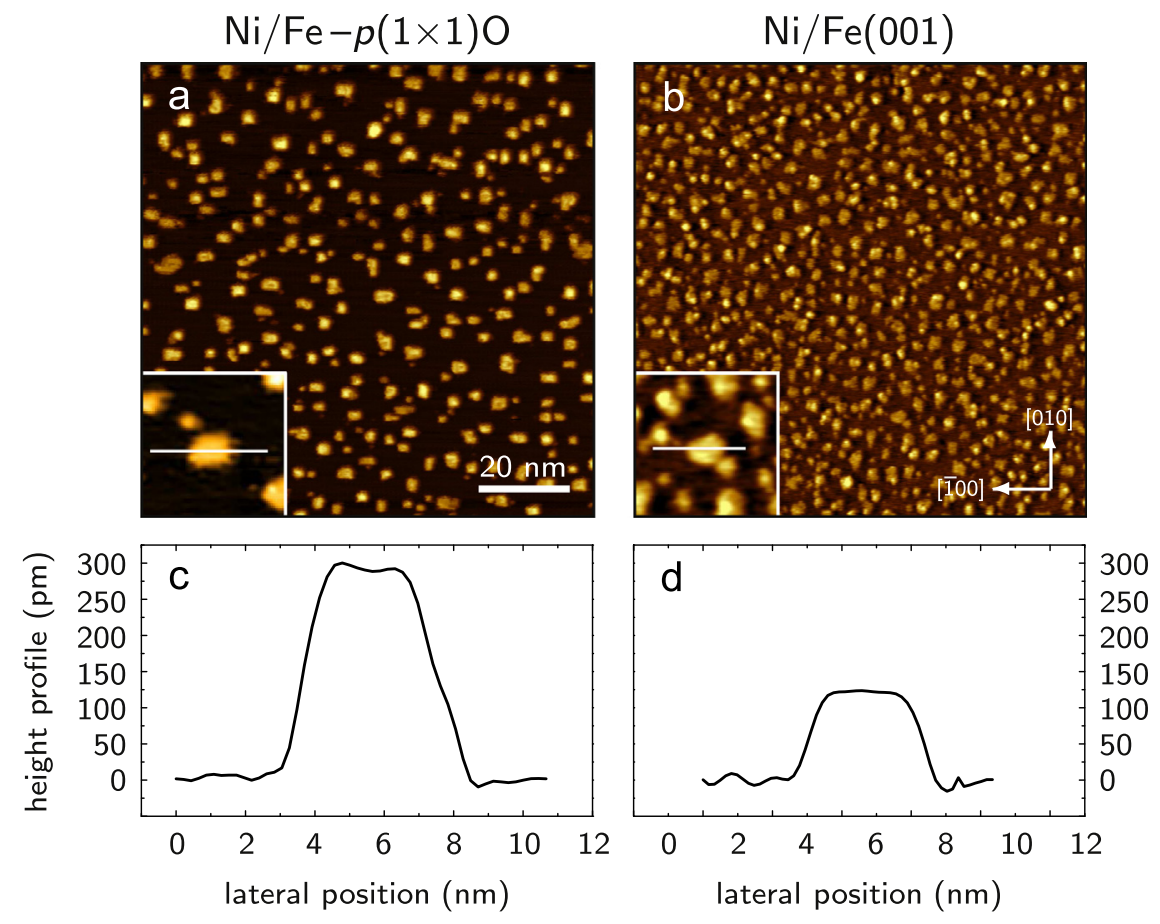

Fig. 22. STM topography after deposition of $0.35 \mathrm{ML}$ of $\mathrm{Ni}$ on (a) $\mathrm{Fe}(001)-p(1 \times 1) \mathrm{O}$ and (b) $\mathrm{Fe}(001)$. (c and d) STM profiles measured along the lines traced in panels (a) and (b), respectively. Reprinted with permission from Ref. [257]. Copyright (2012) by the American Physical Society.
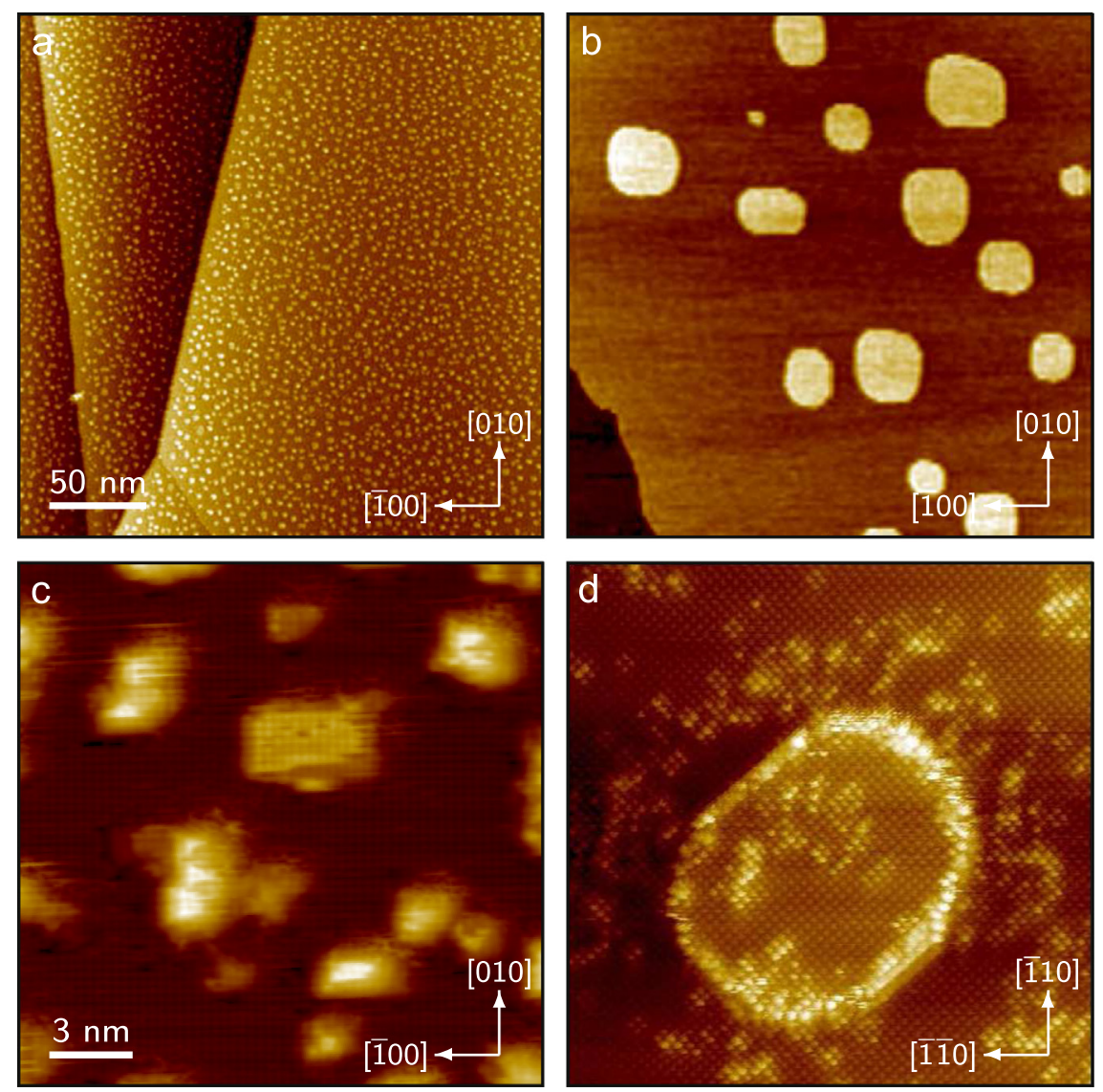

Fig. 23. STM images of $0.6 \mathrm{ML} \mathrm{Ni}$ grown on $\mathrm{Fe}(001)-p(1 \times 1) \mathrm{O}$. (a and c) Morphology of the as-grown sample. (b and d) Morphology after post-annealing treatment at $600 \mathrm{~K}$. The islands size after the annealing increases by one order of magnitude with respect to the case before annealing. (d) Enlarged view of an island: the small bright features on the substrate and on the islands, with apparent height of $40 \mathrm{pm}$, indicate intermixing between $\mathrm{Ni}$ and $\mathrm{Fe}$. Panels (a) and (b): $270 \times 270 \mathrm{~nm}^{2}$; panels (c) and (d): $19 \times 19 \mathrm{~nm}^{2}$. Reprinted with permission from Ref. [257]. Copyright (2012) by the American Physical Society. 

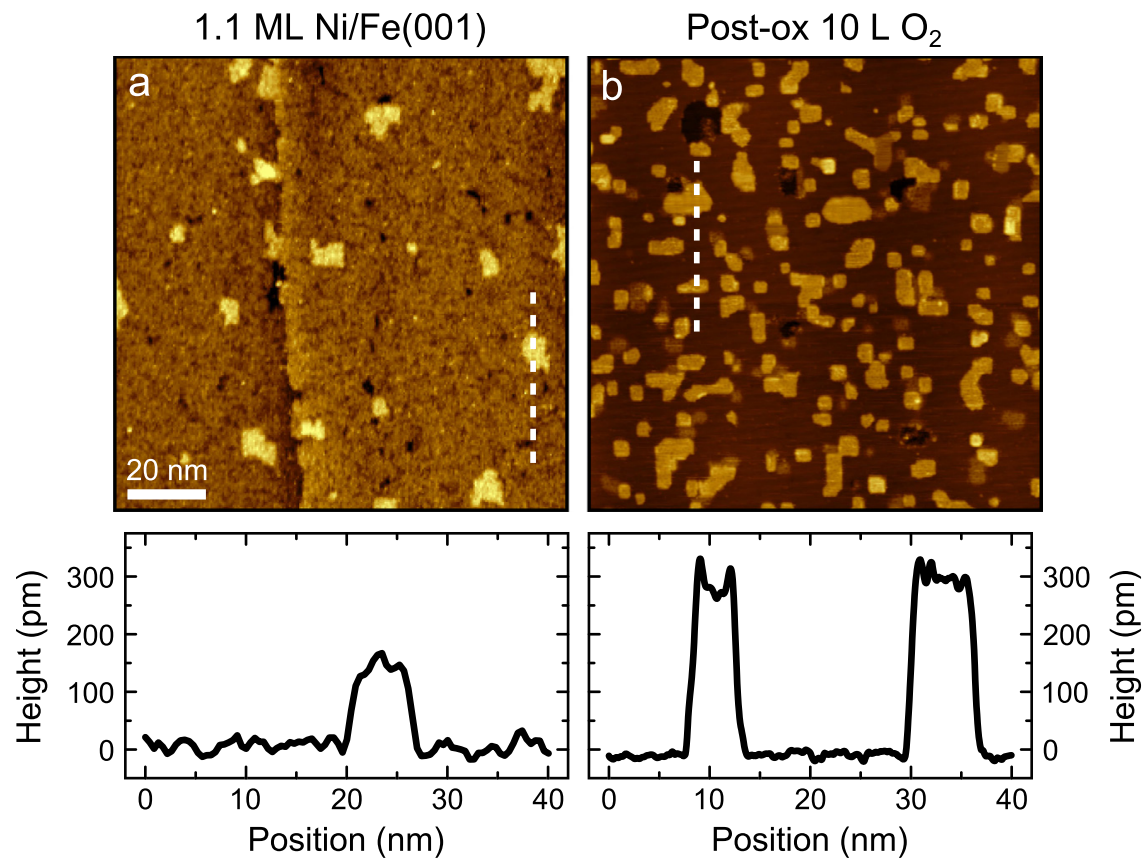

Fig. 24. Effects of the post-oxidation process $\left(10 \mathrm{~L}\right.$ of $\mathrm{O}_{2}$ at $\mathrm{RT}$ and post-annealed to $470 \mathrm{~K}$ for $5 \mathrm{~min}$ ) performed on a $1.1 \mathrm{ML}$-thick Ni film grown at $370 \mathrm{~K}$ on the oxygen-free $\mathrm{Fe}(001)$. Panels (a) and (b) display the surface topography before and after the post-oxidation treatment, respectively. Line profiles along the corresponding dashed white lines are reported at the bottom of each panel. Panel (a) $V=100 \mathrm{mV}, I=100 \mathrm{pA}$; panel (b) $V=1 \mathrm{~V}, I=300 \mathrm{pA}$.

also testified by the faint $c(4 \times 2)$ superstructure visible in the LEED pattern of Fig. 17(d). At a coverage of $0.75 \mathrm{ML}$, the substrate is completely covered by an atomically flat wetting layer characterized by a $c(4 \times 2)$ periodicity, arising from an ordered array of dark holes with a rhombic primitive cell, well-visible in the STM image and LEED pattern reported in Fig. 17(e) and (f), respectively [251]. Increasing the coverage up to $0.8 \mathrm{ML}$ leads to a large-scale reorganization of the overlayer, resulting in a transition toward a $(\sqrt{5} \times \sqrt{5}) R 27^{\circ}$ phase, displayed in the real and reciprocal space in Fig. 17(g) and (h), respectively.

Further insights into the $\mathrm{Cr} / \mathrm{Fe}(001)-p(1 \times 1) \mathrm{O}$ surface and interface chemistry can be acquired from AES spectroscopy. Fig. 18(a) and (b) shows low and high kinetic energy AES spectra, respectively. The spectrum measured on $\mathrm{Fe}(001)-p(1 \times 1) \mathrm{O}$ differs from that of oxygen-free $\mathrm{Fe}(001)$ by the presence of a shoulder located at lower kinetic energy with respect to the metallic peak located at $47 \mathrm{eV}$, readily associated to the presence $\mathrm{Fe}-\mathrm{O}$ bonds (see Section 4.1). After Cr deposition, the shoulder disappears, indicating that the $\mathrm{Fe}-\mathrm{O}$ bonds are broken. However, the peak related to O KLL transitions is still visible in the high kinetic energy region, revealing that oxygen atoms are still present on the surface layer. By combining these pieces of informations it is possible to deduce that the $\mathrm{Fe}-\mathrm{O}$ bonds are replaced by $\mathrm{Cr}-\mathrm{O}$ bonds [251].

Further insight concerning the wetting-layer $\mathrm{Cr}$ oxides come from theoretical analysis, based on DFT simulations performed with the generalized gradient approximation for the exchange and correlation functional as proposed by Perdew, Burke, and Ernzerhof (GGA-PBE) [255]. Fig. 19 presents some structural models based on the $\mathrm{O}-\mathrm{Cr}-\mathrm{Fe}(001)$ stacking suggested by AES spectra. $\mathrm{Cr}$ atoms are located in the hollow sites of the
$\mathrm{Fe}(001)$ surface, originally occupied by oxygen atoms. The latter are placed on Fe-atop sites, between $\mathrm{Cr}$ atoms and slightly above them. The corresponding formation energies per $\mathrm{Cr}$ atom are reported in Table 5.

For a $0.75 \mathrm{ML}-\mathrm{Cr}$ coverage, the most stable arrangement is found to be the $c(4 \times 2)$ structure, with the topmost layer composed by $75 \%$ of $\mathrm{Cr}$ atoms and $25 \%$ by vacancies [see Fig. 19(a)]. This is in good agreement with the observation of the $c(4 \times 2)$ superstructure in the early nucleation stages. Arranging the same amount of $\mathrm{Cr}$ atoms in a $p(2 \times 2)$ overlayer results in a less stable structure by $0.07 \mathrm{eV}$ per $\mathrm{Cr}$ atom. The observed STM images and LEED patterns, displaying the $c(4 \times 2)$ superstructure, could be compatible also with the models of Fig. 19(c) and (d). In the first case, the $\mathrm{Cr}$ magnetic moments are supposed to be alternately parallel/ antiparallel to that of $\mathrm{Fe}$, resulting in a $p(2 \times 4)$ superstructure, where the net magnetization of the $\mathrm{Cr}$ oxide overlayer is zero. However, the strong magnetic coupling with the Fe substrate makes this magnetic configuration less convenient (by $0.13 \mathrm{eV}$ per $\mathrm{Cr}$ atom) with respect to the one where all the $\mathrm{Cr}$ spins antiparallel to the Fe magnetization. In the second case, the dark holes forming the $c(4 \times 2)$ superstructure are supposed to be filled with $\mathrm{Fe}$ atoms. However, in this case the formation energy is highly unfavorable, with an estimated energy cost per $\mathrm{Fe}$ atom equal to $1.27 \mathrm{eV}$.

The $c(4 \times 2)$ phase is characterized by two inequivalent $\mathrm{Cr}$ atoms, denoted by $\mathrm{Cr}_{\mathrm{A}}$ and $\mathrm{Cr}_{\mathrm{B}}$ in Fig. 20(a), possessing three and two nearest $\mathrm{Cr}$ neighbors, respectively. The lower number of $\mathrm{Cr}$ neighbors makes the $\mathrm{Cr}_{\mathrm{B}}$ atoms less affected by magnetic frustration, resulting in a higher magnetic moment for $\mathrm{Cr}_{\mathrm{B}}$ $\left(3.1 \mu_{\mathrm{B}}\right)$ with respect to that of $\mathrm{Cr}_{\mathrm{A}}\left(2.9 \mu_{\mathrm{B}}\right)$. Lowdin population analysis reveals that $\mathrm{Cr}_{\mathrm{A}}$ and $\mathrm{Cr}_{\mathrm{B}}$ differ also by their net 
a
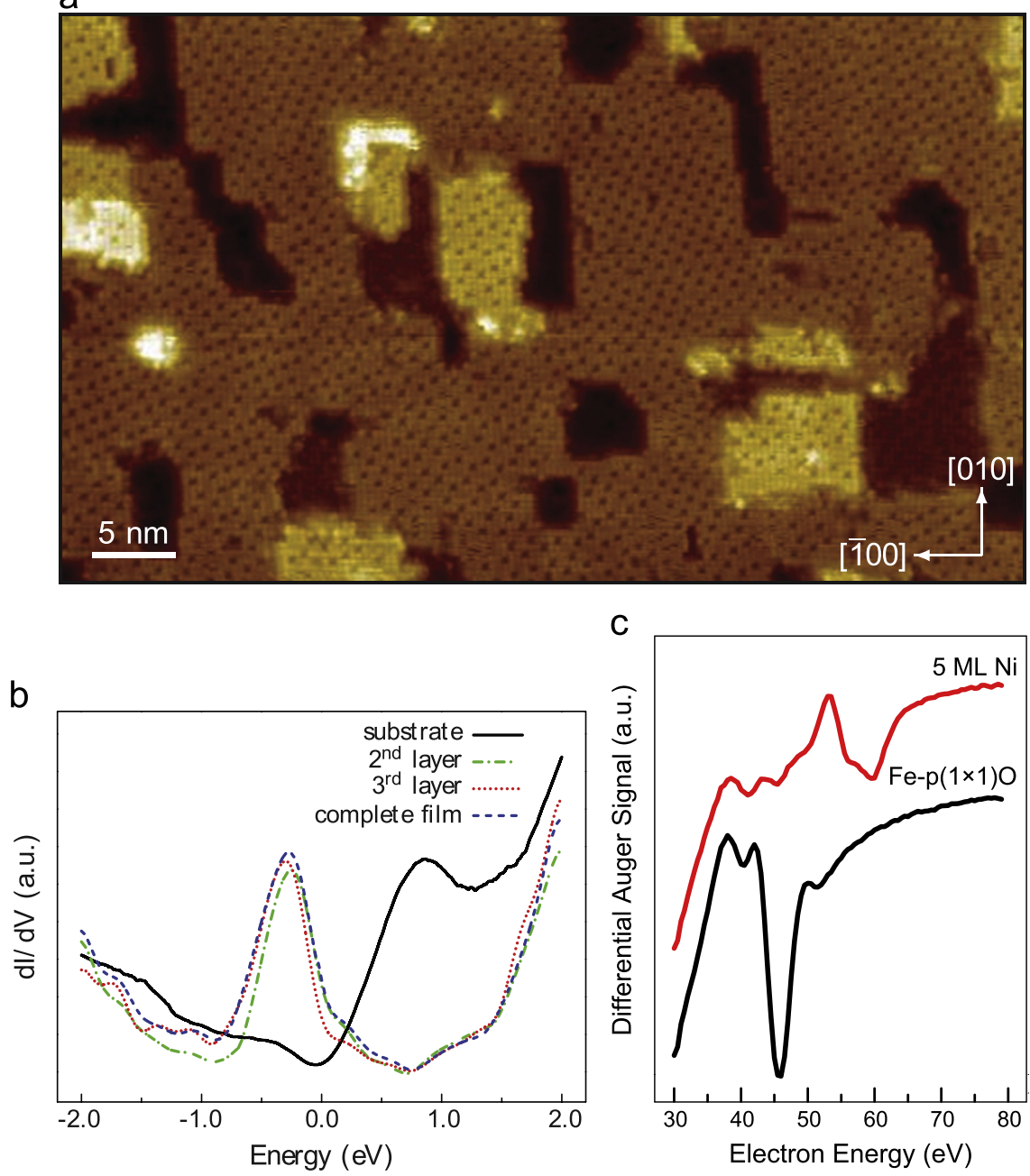

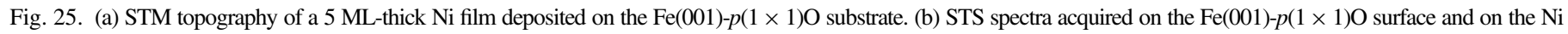

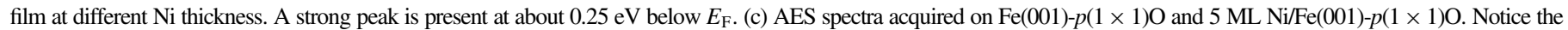

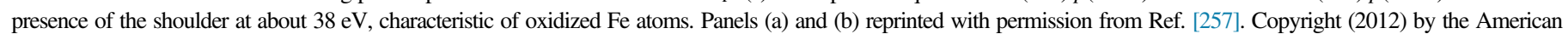
Physical Society.

charge, which are $+1.12 e$ and $+1.16 e$, respectively. Electrons displaced from $\mathrm{Cr}$ atoms mainly move towards the oxygen atoms, which are negatively charged $(-0.62 e$ each). A small fraction of charge also flows towards the first Fe layer $(-0.08 e$ per $\mathrm{Fe}$ atom). The charge flow towards the metallic substrate induces a rumpling of the oxide film, with the oxygen sublayer at larger distances from the support than the cation one [256].

At a coverage of $0.80 \mathrm{ML}$ the energetically most stable structure is $(\sqrt{5} \times \sqrt{5}) R 27^{\circ}$, with a formation energy of $-1.02 \mathrm{eV}$ per atom. The experimentally observed abrupt phase transition from $c(4 \times 2)$ to $(\sqrt{5} \times \sqrt{5}) R 27^{\circ}$ can be understood by comparing the formation energy of the $(\sqrt{5} \times \sqrt{5}) R 27^{\circ}$ phase with that of a mixed phase, obtained by filling $20 \%$ of the $c(4 \times 2)$ vacancy sites with $\mathrm{Cr}$ atoms to obtain $0.80 \mathrm{ML}$ coverage. In this case $25 \%$ of the $\mathrm{Cr}$ atoms would form $p(1 \times 1)$ patches within the $c(4 \times 2)$ phase, yielding $F_{\text {mix }}=0.75 F(\mathrm{a})+0.25 F(\mathrm{~g})=-0.98 \mathrm{eV}$. In the $(\sqrt{5} \times \sqrt{5}) R 27^{\circ}$, all $\mathrm{Cr}$ atoms are equivalent, with a Lowdin charge equal to $+1.12 e$ and a magnetic moment of $3.0 \mu_{\mathrm{B}}$. Similarly to the $c(4 \times 2)$ case, the occupation of vacancy sites by $\mathrm{Fe}$ atoms would increase significantly the formation energy by $1.34 \mathrm{eV}$ per added Fe atom.

The analysis of XMCD profiles extracted from experimental XAS spectra provides information about the magnetization of the different $\mathrm{Cr}$ oxide phases stabilized on the $\mathrm{Fe}(001)$ surface [252]. Fig. 21 reports XAS spectra acquired on the $\mathrm{Cr}_{x} \mathrm{O}_{y}$ layers at coverages of $0.75 \mathrm{ML}[c(4 \times 2)$ phase], and $0.8 \mathrm{ML}$ $\left[(\sqrt{5} \times \sqrt{5}) R 27^{\circ}\right.$ phase] [252]. In both spectra the overlayer dichroic signal is opposite to that of the iron substrate, confirming the theoretic predictions of $\mathrm{Cr}$ oxide wetting layers possessing a ferromagnetic order, antiferromagnetically coupled to the $\mathrm{Fe}(001)$ substrate.

Unlike the $\mathrm{Cr}_{x} \mathrm{O}_{y} / \mathrm{Fe}(001)$ case, it is not possible to stabilize a wetting $\mathrm{NiO}$ layer on the $\mathrm{Fe}(001)$ surface by depositing $\mathrm{Ni}$ on the $\mathrm{Fe}(001)-p(1 \times 1) \mathrm{O}$ substrate. Fig. 22 compares the morphology resulting from $\mathrm{Ni}$ deposition on the $\mathrm{Fe}(001)-p(1 \times 1) \mathrm{O}$ [panel (a)] and $\mathrm{Fe}(001)$ [panel (b)] surfaces, with the substrates held at $400 \mathrm{~K}$ 

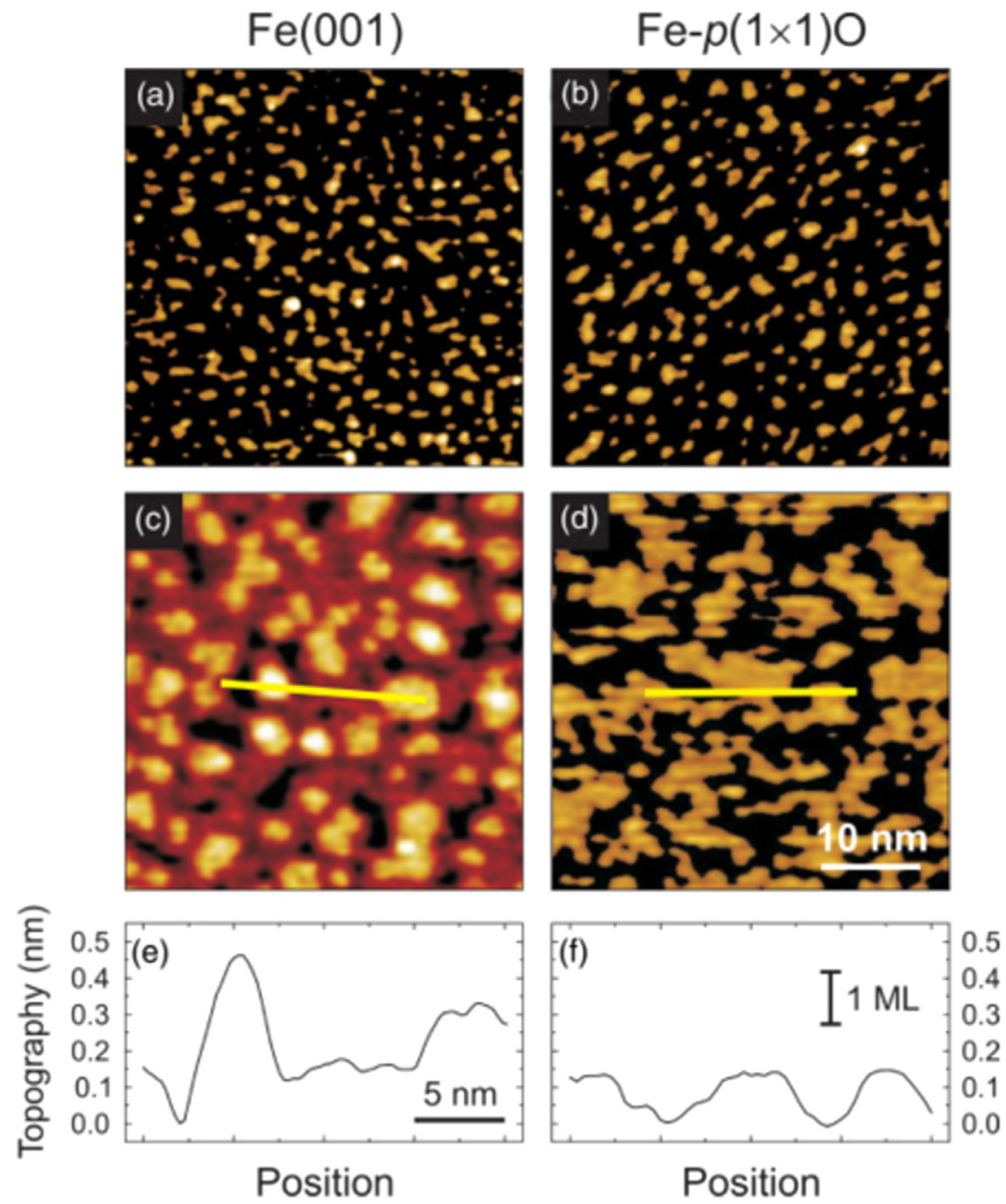

Fig. 26. STM topography after deposition of $0.25 \mathrm{ML}$ of iron on (a) $\mathrm{Fe}(001)$ and (b) $\mathrm{Fe}(001)-p(1 \times 1) \mathrm{O}$. STM topography after deposition of $3.5 \mathrm{ML}$ of iron on (c) $\mathrm{Fe}(001)$ and (d) $\mathrm{Fe}(001)-p(1 \times 1) \mathrm{O}$. (e, f) STM profiles measured along the lines traced in panels (c) and (d), respectively. The image size in panels (a)-(d) is $40 \times 40 \mathrm{~nm}^{2}$. Reprinted with permission from Ref. [223]. Copyright (2011) by the American Physical Society.

during Ni deposition [257]. The STM topographic heights acquired across a $\mathrm{Ni}$ island nucleated on the $\mathrm{Fe}(001)-p(1 \times 1) \mathrm{O}$ and on the Fe(001) substrates are displayed in Fig. 22(c) and (d), respectively. While $\mathrm{Ni}$ deposited on clean $\mathrm{Fe}(001)$ surface nucleates single-layer islands (with a topographic height of about $140 \mathrm{pm}$ ), only bi-layer islands (with a topographic height of about $280 \mathrm{pm})$ are present when $\mathrm{Ni}$ is deposited on the $\mathrm{Fe}(001)-p(1 \times 1) \mathrm{O}$ substrate, suggesting that a single layer of $\mathrm{NiO}$ accommodated on top of $\mathrm{Fe}(001)$ is not stable.

$\mathrm{Ni}$ deposition at higher temperature or post annealing treatments up to $550 \mathrm{~K}$ do not induce a flattening of the bilayer $\mathrm{Ni}$ islands [257]. Instead, post annealing treatments at higher temperatures induce a drastic modifications on the surface morphology. Fig. 23 displays the effect of post annealing at $600 \mathrm{~K}$, performed on a $0.6 \mathrm{ML}$ Ni film deposited on the $\mathrm{Fe}(001)-p(1 \times 1) \mathrm{O}$ surface. The morphology of the asgrown sample, displayed in Fig. 23(a) and (c), is remarkably different compared to that obtained after heating, displayed in Fig. 23(b) and (d). The heating induces the development of larger islands, characterized by an height of $140 \mathrm{pm}$, typical of single layer islands. However, a closer look at the surface reveals the presence of small atomic-scale features, characterized by an apparent height of $40 \mathrm{pm}$, distributed on both the substrate and the islands. This observation suggests that the islands are mainly composed by $\mathrm{Fe}$, while the bright spots are alloyed $\mathrm{Ni}$ atoms.

The instability of the $\mathrm{NiO} / \mathrm{Fe}(001)$ surface is confirmed by the experiment displayed in Fig. 24. In this case a single layer of Ni deposited on the oxygen-free $\mathrm{Fe}(001)$ is exposed to $10 \mathrm{~L}$ of molecular oxygen, and annealed at $470 \mathrm{~K}$ for few minutes. Fig. 24(a) shows the STM topography obtained after deposition of 1.1 ML of $\mathrm{Ni}$ on the $\mathrm{Fe}(001)$ substrate. Ni perfectly wets the $\mathrm{Fe}(001)$ surface, and few islands belonging to the second layer are visible. Fig. 24(b) displays the STM topography obtained after oxygen exposure and post annealing at $470 \mathrm{~K}$. The wetting layer breaks in bi-layer islands covering approximately half of the surface, as in the case of $\mathrm{Ni}$ deposited directly onto the oxygen pre-covered surface.

Notice that the occurrence of bi-layer islands has been seldom reported in metal heteroepitaxy. When observed it was 


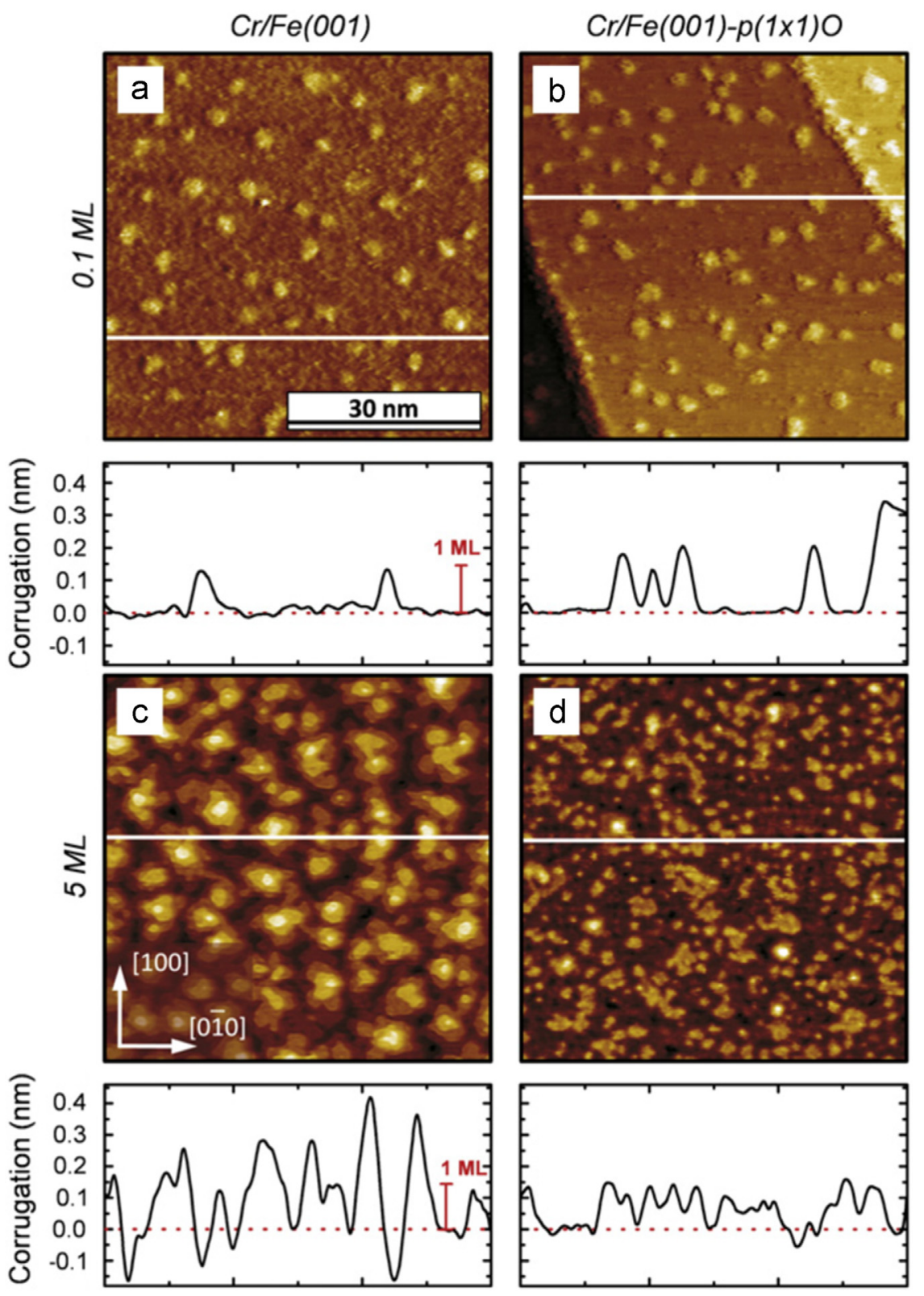

Fig. 27. Comparison of Cr nucleation on $\mathrm{Fe}(001)$ [panels (a) and (c)] and on $\mathrm{Fe}(001)-p(1 \times 1) \mathrm{O}$ [panels (c) and (d)]. The substrate temperature during Cr deposition is $380 \mathrm{~K}$. The Cr coverage is $0.1 \mathrm{ML}$ in panels (a) and (b) and $5 \mathrm{ML}$ in panels (c) and (d). Reprinted with permission from Ref. [278]. Copyright (2011) by Elsevier.

associated to the strain induced by the lattice mismatch. However, for $\mathrm{Ni} / \mathrm{Fe}(001)-p(1 \times 1) \mathrm{O}$, strain does not seem to be the driving force for the nucleation of 2 ML-thick islands. The amount of oxygen present on the $\mathrm{Fe}(001)-p(1 \times 1) \mathrm{O}$ surface is exactly what is needed to form a single layer of $\mathrm{NiO}$. By allowing for a $45^{\circ}$ rotation of the $\mathrm{NiO}$ fcc lattice with respect to the $\mathrm{Fe}$ bcc one, the misfit at the $\mathrm{NiO}(001) / \mathrm{Fe}(001)$ interface is only $3 \%$. Theoretical calculations predict for the single layer of $\mathrm{NiO}$ an even better matching with the $\mathrm{Fe}$ unit cell ( $\mathrm{Ni}-\mathrm{Ni}$ separation in $\mathrm{NiO}$ single layer is close to $2.8 \AA$ ) [258]. In this respect, the interface chemistry seems to play a fundamental role for the instability of a single layer of $\mathrm{NiO}$ on top of $\mathrm{Fe}(001)$.
Although the oxygen preloaded on the substrate does not allow for the stabilization of a single layer $\mathrm{NiO}$, at higher coverages, when the Ni film completely covers the substrate, AES, STM, and STS data suggest that a single layer of a ternary oxide $\mathrm{Ni}_{x} \mathrm{Fe}_{y} \mathrm{O}$ is formed at the surface. Fig. 25 displays the STM, STS and AES data acquired on a 5 ML-thick Ni film grown on $\mathrm{Fe}(001)-p(1 \times 1)$ $\mathrm{O}$. AES spectra in the high kinetic energy region (not shown, see [257]) reveal that oxygen floats on top of the Ni film grown on the $\mathrm{Fe}(001)-p(1 \times 1) \mathrm{O}$ substrate. Atomically resolved STM images, such as that displayed in Fig. 25(a), reveal the presence of a $(\sqrt{5} \times \sqrt{5}) R 27^{\circ}$ reconstruction, indicating that the overlayer is not formed by a single layer of NiO. In the low kinetic energy AES spectra of Fig. 25(c) the small shoulder characteristic of O-bound 
$\mathrm{Fe}$ atoms is clearly visible (see Section 4.1), suggesting that the surface layer can be regarded as a $\mathrm{Ni}_{x} \mathrm{Fe}_{y} \mathrm{O}$ single layer. This interpretation is also supported by the presence of a strong peak in STS spectra detected at about $0.25 \mathrm{eV}$ below $E_{\mathrm{F}}$, very similar to the resonance observed in spectra acquired on invar alloys [259].

\subsection{Growth of metallic buffer layers on $\mathrm{Fe}(001)$ and $\mathrm{Fe}(001)$ - $p(1 \times 1) O$}

From the discussion in the previous section, it is clear that exploiting the oxygen preloaded on the substrate prior to metal deposition could be a useful strategy to obtain a sharp interface between a metallic and an oxidic layer only if the goal is to obtain ultra-thin oxide films in the single-layer range. In order to control the chemical, structural and electronic interactions occurring at $\mathrm{O} / \mathrm{Fe}$ interfaces for thicker films, a suitable strategy could consist in the insertion of a buffer layer. Here, in particular, we discuss the growth of metallic ultra-thin buffer layers on the $\mathrm{Fe}(001)$ surface. A suitable metallic buffer layer should possess specific characteristics, such as a good substrate wettability and a smooth surface (in other words, the growth should be layer-by-layer). The first requirement is mandatory since the presence of voids in the buffer layer would allow a direct contact between the oxidizing agent and the substrate, while the second is important since the surface roughness is likely to increase during the oxide deposition, preventing the stabilization of long-range-ordered epitaxial structures.

In this framework, understanding the atomistic mechanisms governing epitaxial growth is crucial to obtain a suitable metallic buffer layer. The thermodynamics description of epitaxy classifies the growth mode in terms of interface and surface free energies (see Section 2.2.2). However, such a description does not take into account the fact that the epitaxial growth is frequently performed in far-from-equilibrium conditions. Atoms impinging on the substrate diffuse over the surface, encountering energy barriers during their motion. The final film morphology is often determined by these kinetic constraints, especially if the substrate is kept at low temperatures (room temperature or below) during the growth of the film [260-263].

In this context, an important role is played by surfactantassisted growth. A surfactant is a small amount of foreign atoms that, being adsorbed on the surface before film deposition, floats on top of the growing film and contributes to determining its morphology. Surfactants often switch the film growth mode from three-dimensional to two-dimensional. From the point of view of thermodynamics, surfactants change the surface and interface energy balance [264], while in the kinetic picture the surfactants action has been explained in terms of their influence on the diffusion rate of adatoms [265267]. In this case, the growth mode is driven by the ratio between the intralayer and interlayer mass transport rates

\footnotetext{
${ }^{1}$ In ref. [257] the $(\sqrt{5} \times \sqrt{5}) R 27^{\circ}$ reconstruction observed on $5 \mathrm{ML}$ $\mathrm{Ni} / \mathrm{Fe}(001)-p(1 \times 1) \mathrm{O}$ was tentatively related to the presence of $\mathrm{Ni}$ vacancies However, the new AES data reported here suggest that the dark spots observed in STM images arise from a chemical contrast between $\mathrm{Fe}$ and $\mathrm{Ni}$ atoms.
}

[265]. The former is related to the diffusion of atomic species over atomically flat terraces, while the latter expresses the rate at which atoms can overcome a step edge and reach either the lower or higher layer. Generally, the energy barrier at the step edge is higher than that on flat surfaces, since adatoms crossing the step edges are required to pass through low-coordination sites. If interlayer mass transport is much less efficient than intralayer mass transport, deposited atoms are likely to be trapped on top of already nucleated islands, favoring the nucleation of a new layer before lower one is completed. This typically leads to the development of a three-dimensional morphology. The presence of a surfactant overlayer can promote layer-by-layer growth by either decreasing the intralayer rate or increasing the interlayer mass transport.

This kinetic description is certainly the most appropriate one for surfactant-assisted homoepitaxial growth, since the substrate and the deposit possess the same surface free energy. In the case of $\mathrm{Fe} / \mathrm{Fe}(001)$ homoepitaxy, it has been found that $\mathrm{Fe}$ films deposited on the oxygen-free $\mathrm{Fe}(001)$ substrate are characterized by a surface roughness which increases with coverage [268], while the growth on the oxygen-passivated surface is nearly layer-by-layer [269,270,223]. STM images displayed in Fig. 26(a) and (b) reveal that a sub-monolayer amount of Fe deposited on either $\mathrm{Fe}(001)$ or $\mathrm{Fe}(001)-p(1 \times 1) \mathrm{O}$ features the same morphology. In both cases, single-layer islands nucleate, distributed over the surface with basically the same island density, $0.18 \mathrm{~nm}^{-2}$ and $0.16 \mathrm{~nm}^{-2}$ on $\mathrm{Fe}(001)$ and $\mathrm{Fe}(001)-p(1 \times 1) \mathrm{O}$, respectively [223]. Conversely, at higher coverages the surface morphology is quite different. For instance, for 3.5 ML of iron deposited on $\mathrm{Fe}(001)$ four layers are exposed [Fig. 26(c) and (e)], whereas, for the same coverage, iron films grown on $\mathrm{Fe}(001)-p(1 \times 1) \mathrm{O}$ expose only two layers [Fig. 26(d) and (f)]. Oxygen floats on top of the growing Fe film and promotes the development of a smooth surface.

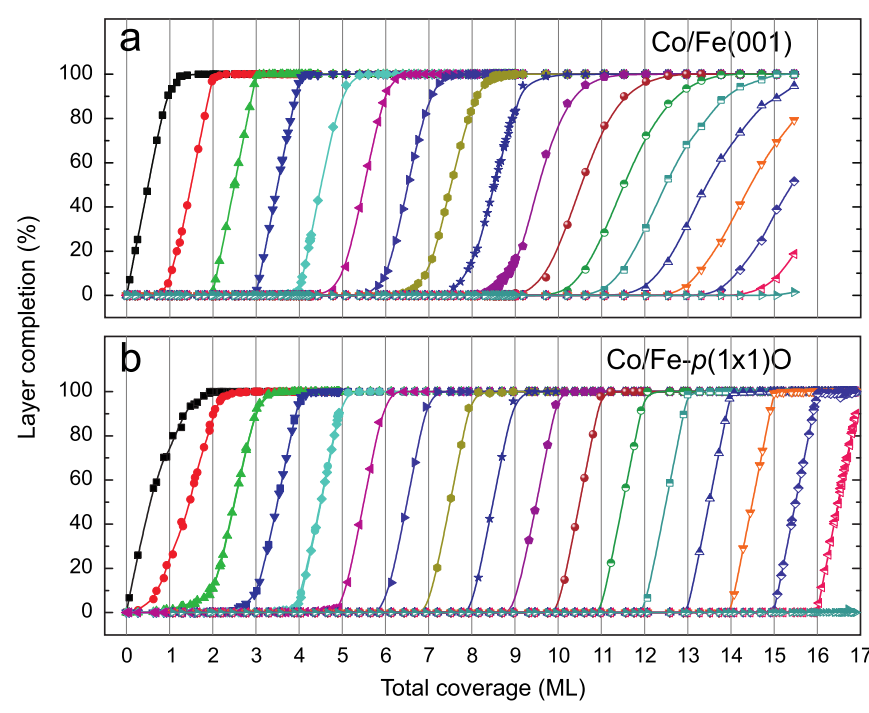

Fig. 28. Layer completion as a function of the total Co coverage on (a) oxygen-free $\mathrm{Fe}(001)$ and on (b) $\mathrm{Fe}(001)-p(1 \times 1) \mathrm{O}$. Reprinted with permission from Ref. [273]. Copyright (2015) by the American Physical Society. 

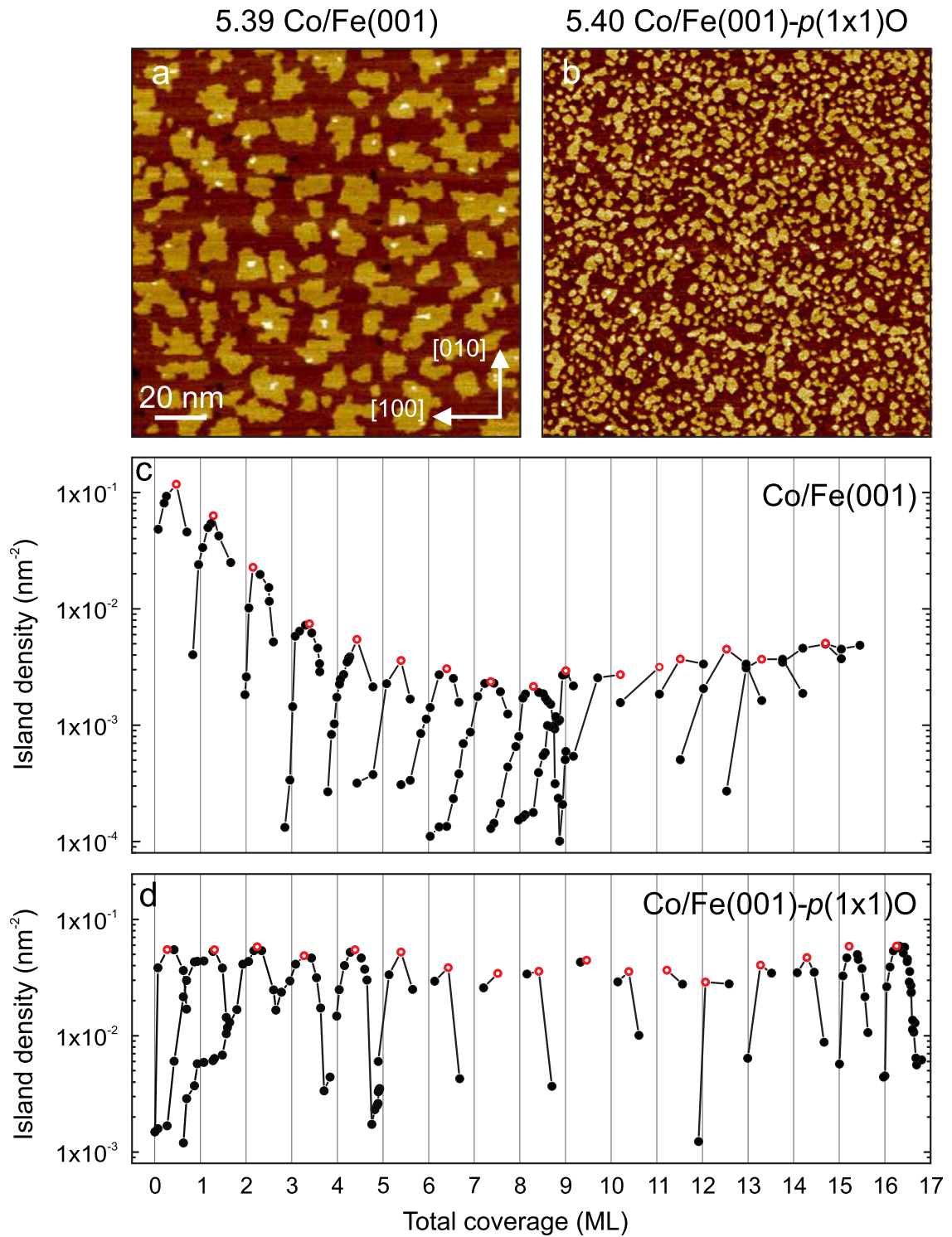

Fig. 29. (a) STM topographic image of a 5.4 ML-thick Co film grown on the oxygen-free Fe(001) surface. (b) STM topographic image of a 5.4 ML-thick Co film grown on the oxygen-passivated $\mathrm{Fe}(001)-p(1 \times 1) \mathrm{O}$ surface. (c and d) Co island density as a function of the total coverage for Co films grown on (c) oxygen-free $\mathrm{Fe}(001)$ and on $(\mathrm{d}) \mathrm{Fe}(001)-p(1 \times 1) \mathrm{O}$, respectively. The local maxima (hollow red dots) correspond to the saturation island density for a given layer. Reprinted with permission from Ref. [273]. Copyright (2015) by the American Physical Society. (For interpretation of the references to color in this figure caption, the reader is referred to the web version of this paper.)

In order to understand the surfactant action of oxygen we recall that, according to the classical nucleation theory [260], the island density is directly related to the atomic diffusion rate over the surface (intra-layer mass transport). Generally, the nucleation of large islands distributed with a low density over the surface indicates a high diffusion rate of atomic species, while the nucleation of a high density of small islands is observed in the case of low diffusion rates. Since the islands density is nearly the same for sub-monolayer deposition on both the oxygen-free and oxygen-passivated surface, the oxygen surfactant action in Fe homoepitaxy can be understood as a consequence of an oxygen-induced lowering of the energy barrier for interlayer mass transport [223]. Notice that the invariance of island density on the oxygen-free and oxygenpassivated surfaces represents a far-from-trivial observation, since the presence of surfactant atoms has been found to have a direct impact on the intralayer mass transport in a considerable number of cases, typically by enhancing the nucleation rate [271,272,265,273].

The oxygen surfactant action is found to be effective also for the growth of heteroepitaxial films, as demonstrated for instance in the case of oxygen-assisted growth of $\mathrm{Mn}$ on $\mathrm{Fe}$ [274], $\mathrm{Fe}, \mathrm{Co}$ and $\mathrm{Ni}$ on $\mathrm{Cu}$ [275-277]. Fig. 27 shows an example of oxygen-assisted heteroepitaxial growth of $\mathrm{Cr}$ films deposited on the $\mathrm{Fe}(001)$ surface [278]. Similar to the case of $\mathrm{Fe} / \mathrm{Fe}(001)$ homoepitaxy, the density of $\mathrm{Cr}$ islands nucleating during the early stages of growth on the $\mathrm{Fe}(001)$ [Fig. 27(a)] and $\mathrm{Fe}(001)-p(1 \times 1) \mathrm{O}$ [Fig. 27(b)] surfaces is about the same, suggesting a negligible effect of the oxygen overlayer on the intralayer mass transport of $\mathrm{Cr}$ atoms. At higher 

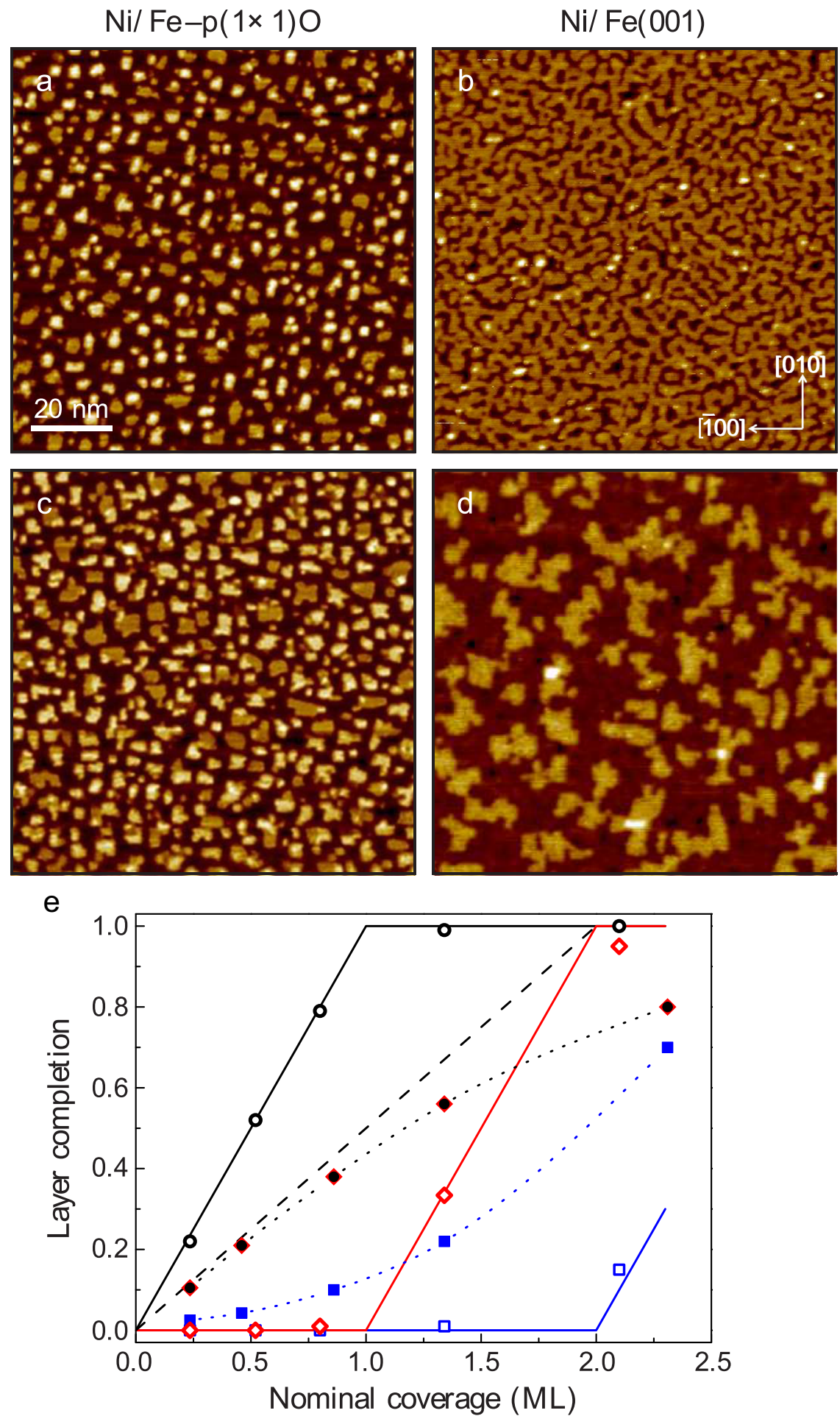

Fig. 30. STM topography after deposition of (a) 0.85 or (c) $1.35 \mathrm{ML}$ of $\mathrm{Ni}$ on $\mathrm{Fe}(001)-p(1 \times 1) \mathrm{O}$, and (b) 0.85 or (d) $1.35 \mathrm{ML}$ of $\mathrm{Ni}$ on Fe(001). (e) Layer completion fraction vs. the number of equivalent monolayers of deposited Ni. The first layer filling is indicated by black circles, the second by red diamonds, and the third layer by blue squares. Full (open) symbols are used for Ni growth on the $\mathrm{Fe}(001)-p(1 \times 1) \mathrm{O}$ [Fe(001)] surface. The trend of both an ideal layer-by-layer growth (full lines) and an ideal two layers-by-two layers growth (dashed line) are also reported for comparison. Dotted lines: guide for the eye. Reprinted with permission from Ref. [257]. Copyright (2012) by the American Physical Society. (For interpretation of the references to color in this figure caption, the reader is referred to the web version of this paper.)

coverages, the oxygen overlayer, floating on top of the Cr film, promotes the development of a two-dimensional morphology [see Fig. 27(d)], while Cr deposited on the $\mathrm{Fe}(001)$ surface develops a rough surface [see Fig. 27(c)].
Another example of the influence that a ultra-thin surfactant oxide layer can have on the morphology of heteroepitaxial growth of thin metal layer is provided by the different morphologies exhibited by $\mathrm{Co}$ films grown on either the oxygen-free $\mathrm{Fe}$ 

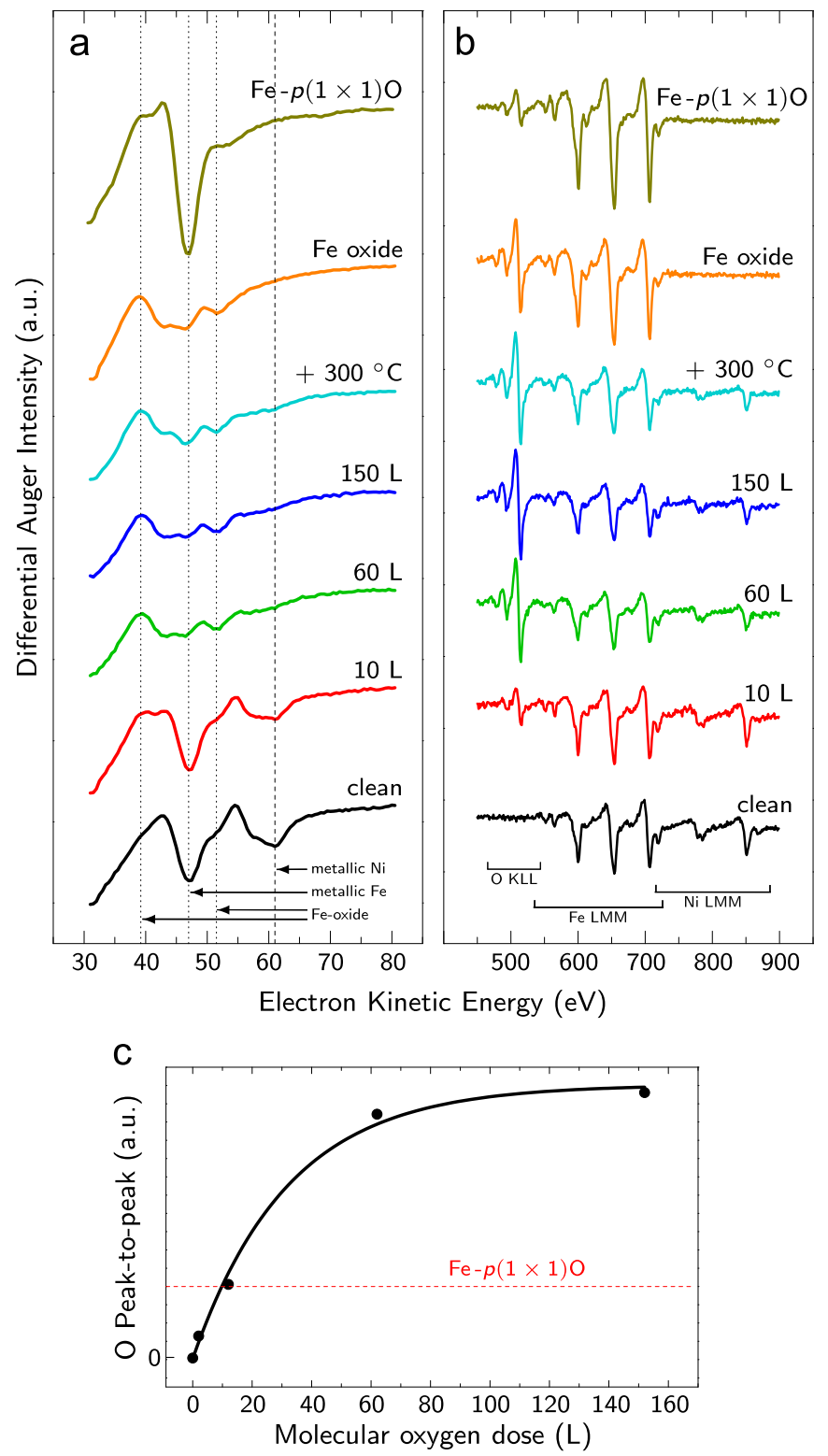

Fig. 31. AES spectra acquired after the oxidation of a 4 ML-thick Ni film deposited on $\mathrm{Fe}(001)$. (a) $\mathrm{Ni}$ and $\mathrm{Fe}$ MNN AES lineshape evolution for increasing oxygen exposure. After each oxygen dosing the sample was annealed for $5 \mathrm{~min}$ at $473 \mathrm{~K}$. The reference spectra collected on the $\mathrm{Fe}(001)-p(1 \times 1) \mathrm{O}$ surface and on a $\mathrm{Fe}(001)$ sample exposed to $50 \mathrm{~L}$ of oxygen are displayed in the upper part of panels (a) and (b). (b) AES spectra acquired in the high-kinetic region on the same samples of panel (a). (c) Oxygen KLL peak-to-peak intensity as a function of the oxygen dose. The intensity measured on the $\mathrm{Fe}(001)-p(1 \times 1) \mathrm{O}$ surface (single layer of oxygen) is indicated by the horizontal dashed red line. Reprinted with permission from Ref. [279]. Copyright (2014) by Elsevier. (For interpretation of the references to color in this figure caption, the reader is referred to the web version of this paper.)

(001) and the oxygen-saturated $\mathrm{Fe}(001)-p(1 \times 1) \mathrm{O}$ surface. In the latter case, AES spectroscopy confirms that $\mathrm{O}$ atoms keep floating on the Co film surface during deposition [273]. Constant-current
STM images reveal that the Co growth on the oxygen-free surface proceeds in a layer-by-layer mode up to about $9 \mathrm{ML}$ [see Fig. 28(a)], after which such a growth mode is destabilized by plastic deformations induced by the relaxation of the strain accumulated in the film [153]. Conversely, when Co is deposited on the $\mathrm{Fe}(001)-p(1 \times 1) \mathrm{O}$, a wetting layer is completed only after a coverage of $2 \mathrm{ML}$, then a nearly perfect layer-by-layer growth is initiated. This proceeds much further with respect to the former case, extending at least up to $17 \mathrm{ML}$ [see Fig. 28(b)].

Further hints about the role of the oxygen overlayer in determining the structural properties of $\mathrm{Co} / \mathrm{Fe}(001)$ or $\mathrm{Co} / \mathrm{Fe}-p(1 \times 1) \mathrm{O}$ are provided by the analysis of the island density as a function of coverage [see Fig. 29]. A Co atom landing on the substrate diffuses over the surface, until it encounters another diffusing atom and forms a dimer that anchors to the substrate, nucleating an island. At higher coverages, the number of nucleated islands per unit area increases up to a critical value. Further deposition of material induces the growth of already present islands, which start merging together. In this way, the density of islands on the layer reaches a maximum (known as "saturation island density"), until a complete wetting layer covers the substrate. The same scenario then repeats for the subsequent layers. In the case of deposition onto oxygen-free $\mathrm{Fe}(001)$, the saturation island density drastically decreases, by almost two orders of magnitude, for increasing Co coverages. Conversely, when Co is evaporated onto $\mathrm{Fe}(001)-p(1 \times 1) \mathrm{O}$, the saturation island density is not affected by the Co film thickness across the $0-17$ ML coverage range.

DFT calculations suggest that the remarkable decrease of the saturation island density on a $\mathrm{Co} / \mathrm{Fe}(001)$ film is due to an enhancement of atomic diffusion driven by the increasing capability of the film to accommodate large deformations as the thickness approaches the limit at which a structural transition occurs [153]. In the case of $\mathrm{Co} / \mathrm{Fe}(001)-p(1 \times 1) \mathrm{O}$, the presence of the oxide overlayer completely change the diffusion mechanisms of Co adatoms, leading to a thicknessindependent diffusion coefficient [273].

An interesting case in which the oxygen overlayer changes the growth mode in the opposite direction, i.e. inducing a three-dimensional morphology when the growth on the oxygen free-surface proceeds in the layer-by-layer mode, has been found in the early stages of $\mathrm{Ni}$ growth on $\mathrm{Fe}(001)-p(1 \times 1) \mathrm{O}$ [257]. As already discussed in Section 4.2, the Ni growth on the oxygen-passivated surface starts by the nucleation of bilayer islands, while single layer islands nucleate over the oxygen-free surface. Fig. 30 focuses on thicker Ni films than those shown in Fig. 24, deposited on either $\mathrm{Fe}(001)-p(1 \times 1) \mathrm{O}$ [panels (a) and (c)] or Fe(001) [panels (b) and (d)]. The layer completion graph, displayed in Fig. 30(e), shows that, when $\mathrm{Ni}$ is deposited on $\mathrm{Fe}(001)-p(1 \times 1) \mathrm{O}$, the third layer develops well-before the completion of the first two, resulting in a threedimensional growth. On the other hand, a nearly perfect layer- 

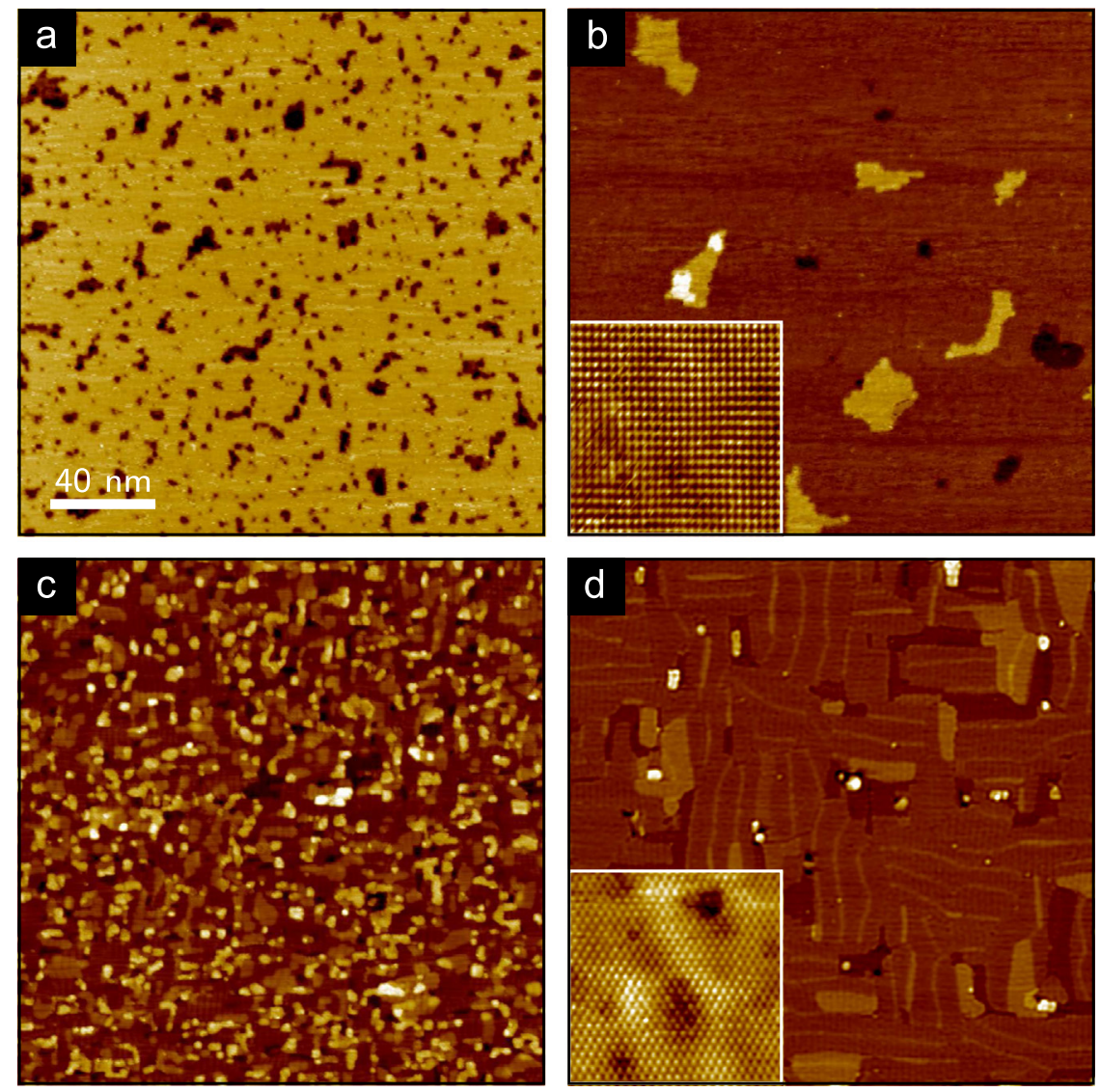

Fig. 32. STM topography of (a) the pristine $4 \mathrm{ML} \mathrm{Ni/Fe}(001)$ surface; (b) the same film after exposure to $10 \mathrm{~L}$ of $\mathrm{O}_{2}$ and annealing at $473 \mathrm{~K}$; (c) the sample of panel (b) dosed with $150 \mathrm{~L}$ of $\mathrm{O}_{2}$ and annealed at $473 \mathrm{~K}$; (d) the sample of panel (c) annealed at $573 \mathrm{~K}$ in UHV conditions. Reprinted with permission from Ref. [279]. Copyright (2014) by Elsevier.

by-layer growth is observed in the case of $\mathrm{Ni}$ deposited on the oxygen-free surface.

\subsection{Oxidation of metallic buffer layers on $\mathrm{Fe}(001)$}

In this section we discuss the effects of oxygen-exposure on metallic buffer layers deposited on $\mathrm{Fe}(001)$. Important phenomena that need to be accounted for to understand these heterostructures include oxygen-induced segregation of atomic species from the substrate to the surface, surface roughening, and interfacial intermixing. In particular, the possibility of stabilizing an oxide by post-deposition oxidation of a metal on $\mathrm{Fe}(001)$ is an issue that bears many points in common with the wetting behavior of ultra-thin oxides on $\mathrm{Fe}(001)$ (see Section 4.2). For instance, while a sharp $\mathrm{NiO} / \mathrm{Fe}(001)$ interface cannot be obtained by either post-oxidation of an $\mathrm{Ni}$ films deposited on $\mathrm{Fe}(001)$ or by exploiting the preloaded oxygen on $\mathrm{Fe}(001)-p(1 \times 1) \mathrm{O}$, chemically stable $\mathrm{Cr}$ ultra-thin oxides on $\mathrm{Fe}(001)$ can be obtained by following both strategies $[279,280]$.

A Ni buffer layer grown on the $\mathrm{Fe}(001)$ surface is not effective in protecting the substrate from oxidation, since oxygen exposure induces the segregation of $\mathrm{Fe}$ atoms towards the surface, where their oxidation takes place. Fig. 31 reports the evolution of AES spectra acquired on $4 \mathrm{ML} \mathrm{Ni} / \mathrm{Fe}(001)$ treated with cycles of room
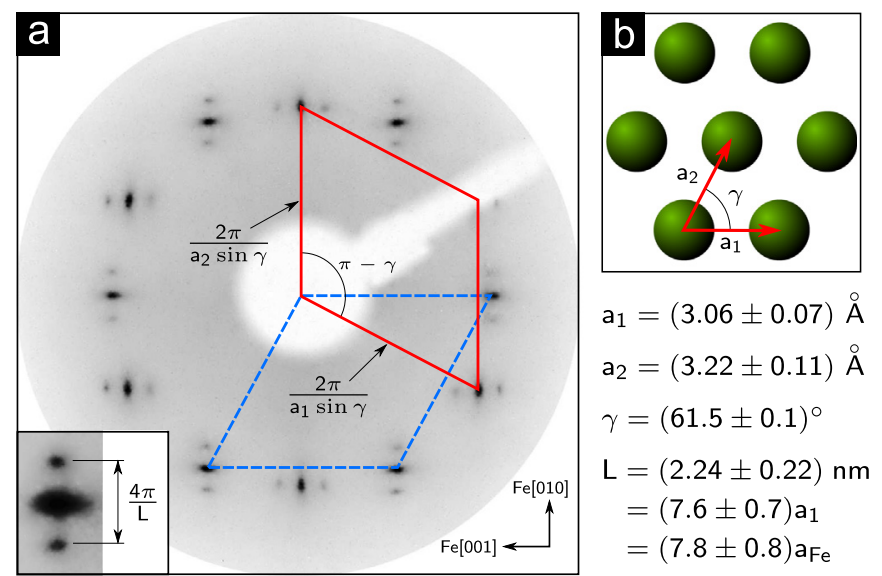

Fig. 33. (a) LEED pattern (primary energy $=100 \mathrm{eV}$ ) of the Ni/Fe sample dosed with $150 \mathrm{~L}$ of oxygen and annealed at $573 \mathrm{~K}$. Two orthogonal rotational quasi-hexagonal domains are visible. The reciprocal unit cells are drawn in red (solid line) and blue (dashed line). A blow-up of one of the spots is reported in the inset, where the contrast has been artificially enhanced to increase the visibility of the mutually orthogonal elongation and spot splitting. The relevant parameters extracted from the pattern to obtain the real-space atomic unit cell (main panel) and the supercell periodicity (inset) are indicated. (b) Real space model atomic Bravais lattice for the quasi-hexagonal overlayer as obtained from the LEED pattern in (a). The numerical values of the parameters are also reported. Reprinted with permission from Ref. [279]. Copyright (2014) by Elsevier. (For interpretation of the references to color in this figure caption, the reader is referred to the web version of this paper.) 
temperature oxygen exposure and annealing in UHV. The freshly deposited ultra-thin Ni films are exposed to an increasing dose of molecular oxygen at room-temperature [oxygen partial pressure during the oxidation from $10^{-9}$ mbar for the lowest exposure and $10^{-7}$ mbar for the highest one (150 L)], and subsequently annealed in UHV at $473 \mathrm{~K}$ for $5 \mathrm{~min}$. Before the oxidation treatment, the low-energy AES spectrum [Fig. 31(a)] of the as-grown sample is characterized by the presence of two main peaks located at $47 \mathrm{eV}$ and $61 \mathrm{eV}$, related to metallic $\mathrm{Fe}$ and $\mathrm{Ni}$, respectively. Upon a $10 \mathrm{~L}$ oxygen exposure, a shoulder appears at lower kinetic energy with respect to the metallic Fe peak. Such a shoulder is analogous to the one present on the $\mathrm{Fe}(001)-p(1 \times 1) \mathrm{O}$ surface (see Section 4.1), suggesting the formation of $\mathrm{O}-\mathrm{Fe}$ bonds. Increasing the oxygen exposure up to $60 \mathrm{~L}$ clearly enhances the intensity of the peaks associated with O-bound $\mathrm{Fe}$ atoms, while the Ni peak almost disappears. Further increase of the oxygen exposure up to $150 \mathrm{~L}$ and the final annealing at $573 \mathrm{~K}$ in UHV do not significantly change the shape and the intensity of the measured peaks. In the high kinetic energy region [Fig. 31(b)], in which the mean free path of electrons is longer, i.e. AES spectra are less surface sensitive, it is possible to notice that the Ni peak intensity does not vanish, indicating that some $\mathrm{Ni}$ is present underneath the $\mathrm{Fe}$ oxide film.

STM topographies corresponding to each oxidation step are displayed in Fig. 32. Before oxidation, Ni films display an atomically smooth surface [Fig. 32(a)], with mainly two layers exposed, as expected for a layer-by-layer growth. Upon exposure to $10 \mathrm{~L}$ of molecular oxygen, the surface retains a flat morphology [Fig. 32(b)]. Thanks to the oxygen-induced increase of the atomic corrugation, atomic resolution is achieved, showing a lattice with square symmetry and in-plane periodicity equal to that of the $\mathrm{Fe}(001)-p(1 \times 1) \mathrm{O}$ surface $(2.87 \AA)$. However, based on AES spectra, this surface atomic layer is expected to be $\mathrm{Fe}$ oxide. Increasing the oxygen exposure to $60 \mathrm{~L}$ promotes surface roughening [Fig. 32(c)]. Annealing the sample at $573 \mathrm{~K}$ in UHV conditions induces the development of large atomically flat terraces, visible in Fig. 32(d). Moreover, the atomically resolved image displayed in the inset of Fig. 32(d) reveals that the unit cell switches from the square symmetry characterizing the early stages of oxidation to the hexagonal one.

Such hexagonal arrangement of surface atoms is also visible in the LEED pattern of Fig. 33(a), revealing the presence of two orthogonal domains, aligned along the main crystallographic directions of the $\mathrm{Fe}(001)$ surface. The analysis of the LEED pattern provides the structural model of Fig. 33(b). The direct-space basis vectors are about $3.06 \AA$ and $3.22 \AA$ long, rotated by an angle of $(61.5 \pm 0.1)^{\circ}$. Being the nearestneighbor spacing between atoms in the $\mathrm{FeO}(111)$ surface equal to $a_{\mathrm{FeO}}=3.04 \AA$ [281], this analysis suggests that the overlayer consists of a $\mathrm{FeO}(111)$ distorted film.

The film surface appears to be modulated, as revealed by the splitting of the LEED diffraction spots as well as by STM images reported in Fig. 34. Two mutually orthogonal modulations are visible, the first one largely variable from about 7 to $15 \mathrm{~nm}$ [see
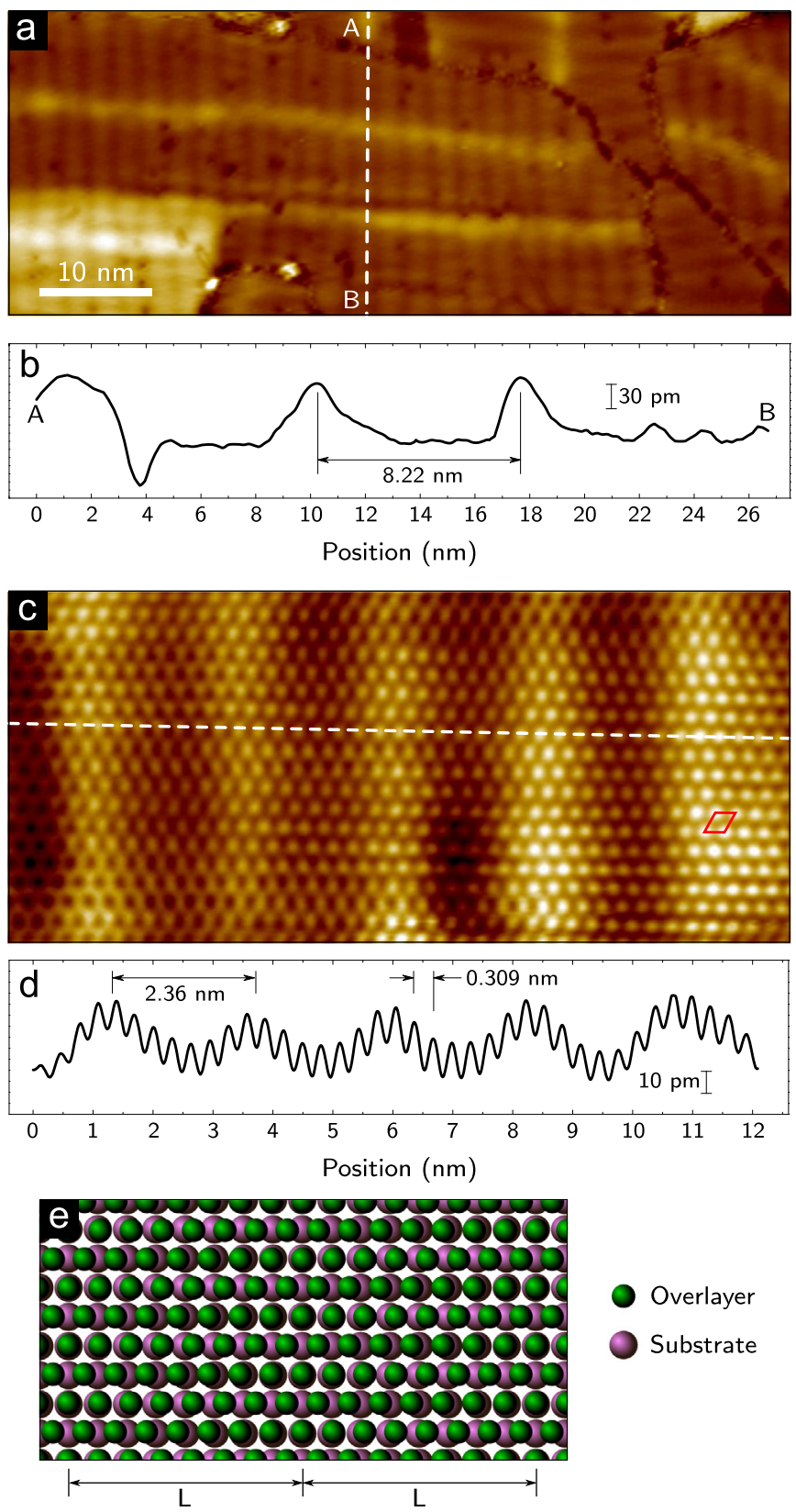

Fig. 34. $4 \mathrm{ML} \mathrm{Ni} / \mathrm{Fe}(001)$ surface dosed with $150 \mathrm{~L}$ of oxygen and heated at $573 \mathrm{~K}$. (a) Large scale STM topography ( $I=1 \mathrm{nA}, V=1 \mathrm{~V})$ showing several rotational domains characterized by the mutually orthogonal surface undulations. (b) Topographic profile acquired along the dashed white line in (a). The peak-topeak distance between the maxima of the longer modulation visible in (a) is indicated. (c) Atomically resolved STM topography $(I=5 \mathrm{nA}, V=0.1 \mathrm{~V})$ of one of the rotational domains, showing the detail of the short-period modulation. The distorted hexagonal atomic unit cell is indicated in red on the right. (d) Height profile along the dashed white line in (c). The modulation periodicity (L) and the $\mathbf{a}_{1}$-unit vector length are indicated (refer to Fig. 33 for the notation). (e) Result of the superposition of the distorted hexagonal lattice (green; unit vectors and relative orientation as measured from the LEED pattern in Fig. 33) and a square lattice (violet) with the same spatial periodicity of $\mathrm{Fe}(001)$. The short-period modulation results from the quasi-coincidence of the two lattices along the $\mathbf{a}_{1}$ direction. Reprinted with permission from Ref. [279]. Copyright (2014) by Elsevier. (For interpretation of the references to color in this figure caption, the reader is referred to the web version of this paper.) 

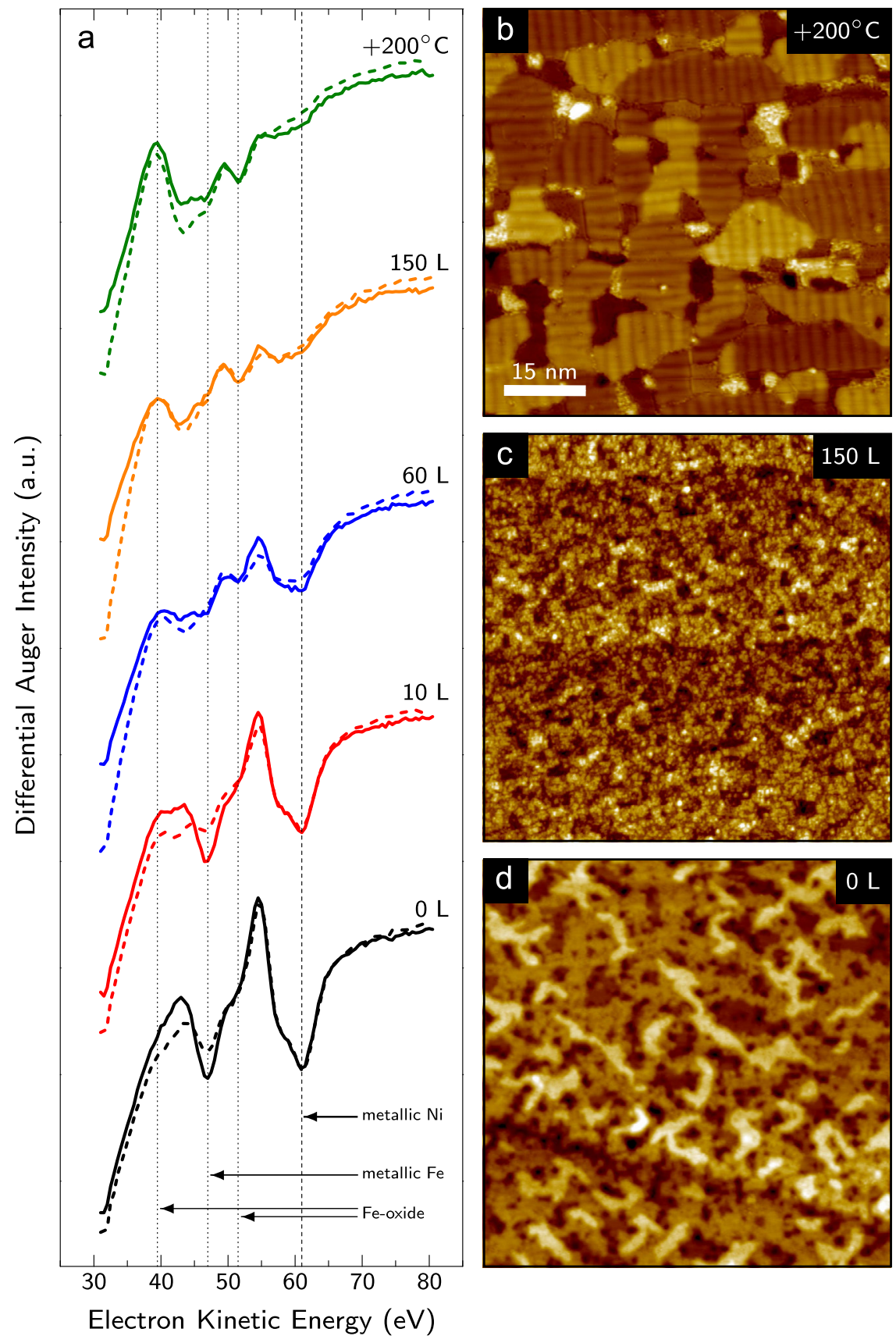

Fig. 35. (a) Low kinetic energy Auger spectra for the $5 \mathrm{ML}$-thick $\mathrm{Ni} / \mathrm{Fe}(001)$ sample at different oxygen exposures. No post-annealing treatment was performed until the highest dose of $150 \mathrm{~L}$ was reached. The topmost spectrum refers to the final sample $(150 \mathrm{~L}$ oxygen) after a mild-temperature annealing. Solid and dashed curves refer to spectra acquired at normal and grazing (sample normal $77^{\circ}$ tilted with respect to the incident electron beam) emissions, respectively. The position of relevant $\mathrm{Ni}$ (dashed) and Fe (dotted) transitions is marked by vertical lines. (b-d) STM images for the sample at a selection of the preparation steps. Namely (d) pristine $\mathrm{Ni} / \mathrm{Fe}(001)$, (c) $150 \mathrm{~L}$ and (b) $150 \mathrm{~L}+$ annealing at $473 \mathrm{~K}$. Reprinted with permission from Ref. [279]. Copyright (2014) by Elsevier.

Fig. 34(a) and (b)] and a second one with a shorter period of $2.36 \mathrm{~nm}$ [see Fig. 34(c) and (d)]. These two mesoscopic-range periodic modulations arise from the superposition of the surface hexagonal lattice with the square lattice of the $\mathrm{Fe}(001)$ surface, resulting in the quasi-coincidence superlattice displayed in Fig. 34(e).

The oxygen-induced Fe segregation is observed also when oxygen is dosed on the $\mathrm{Ni} / \mathrm{Fe}(001)$ sample without any intermediate annealing step. Fig. 35 displays the AES spectra [panel (a)] and the STM morphology [panel (b)] resulting from the oxidation of a $5 \mathrm{ML} \mathrm{Ni} / \mathrm{Fe}(001)$ sample, where the annealing step was performed only on the sample dosed with the highest amount of oxygen $(150 \mathrm{~L})$. The $\mathrm{Fe}$ oxide peak appears after oxygen exposure, being more pronounced for spectra acquired at grazing incidence [dashed spectra in Fig. 35(a)], an observation suggesting that $\mathrm{Fe}$ oxidation takes place in the surface region. The final annealing step increases the amount of the superficial Fe oxide, in line with previous works [42].

STM topography acquired on the $5 \mathrm{ML} \mathrm{Ni/Fe}(001)$ system before oxygen exposure [Fig. 35(d)] reveals that the film 


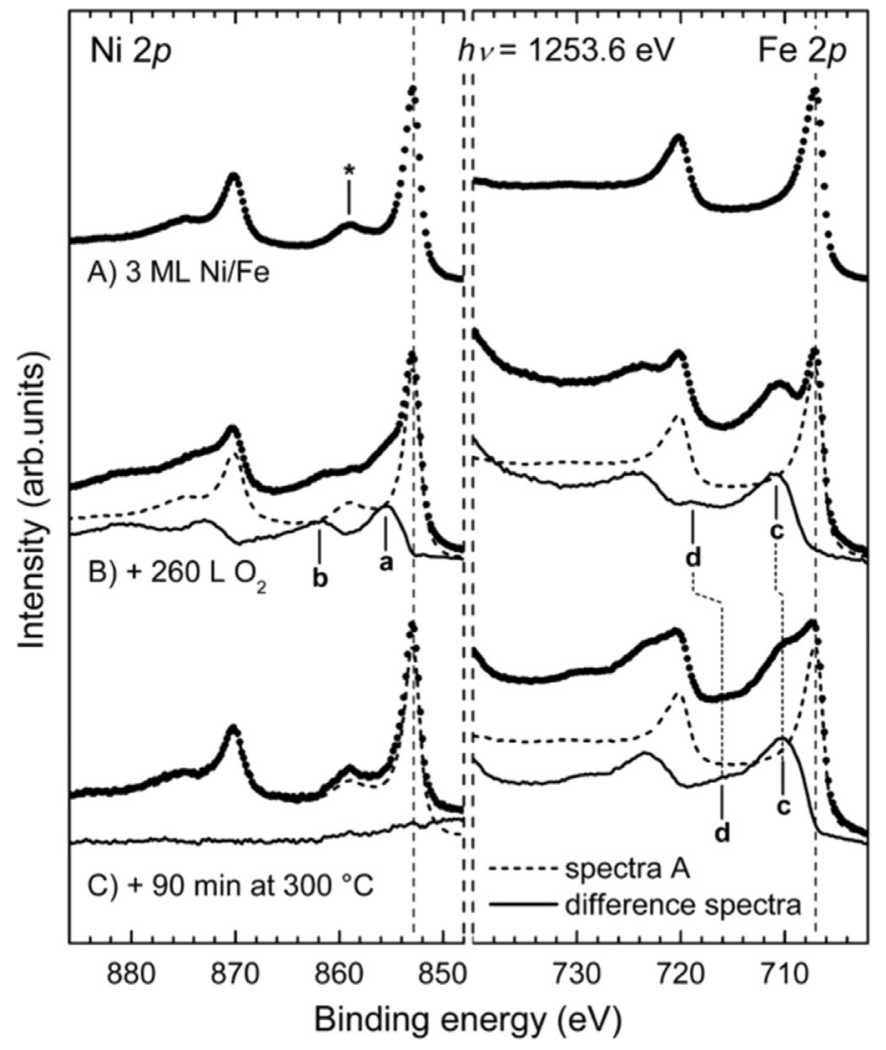

Fig. 36. XPS scans of the Ni $2 p$ and $\mathrm{Fe} 2 p$ regions of a $3 \mathrm{ML} \mathrm{Ni/Fe}(001)$ sample. (A) as-grown, (B) exposed to $260 \mathrm{~L}$ of $\mathrm{O}_{2}$ at room temperature and (C) annealed at $573 \mathrm{~K}$ for $90 \mathrm{~min}$ in UHV. All spectra have been acquired at grazing emission (photoelectrons are emitted with an angle of $60^{\circ}$ with respect to the surface normal) with $\mathrm{Mg} \mathrm{K} \alpha$ radiation. Continuous lines are difference spectra obtained by subtracting the A spectra (dashed lines in spectra B and C) from the experimental data. All spectra have been normalized to the intensity of the as-grown component. Reprinted with permission from Ref. [280]. Copyright (2014) by IOP Publishing.

morphology is slightly rougher with respect to the $4 \mathrm{ML} \mathrm{Ni} / \mathrm{Fe}$ (001) sample. The roughness keeps increasing upon oxygen exposure at room temperature, as visible in the STM image acquired after dosing the sample with $150 \mathrm{~L}$ of $\mathrm{O}_{2}$ [Fig. 35(b)]. Interestingly, the final annealing step again drives the development of the modulated $\mathrm{FeO}(111)$ film [Fig. 35(c)], suggesting that this phase is particularly stable, irrespective of the preparation procedure.

Further insights in the $\mathrm{Ni} / \mathrm{Fe}(001)$ oxidation mechanisms come from XPS and UPS [280]. In this case the oxidation is performed by exposing a $3 \mathrm{ML} \mathrm{Ni} / \mathrm{Fe}(001)$ sample to $\mathrm{O}_{2}$. Fig. 36 displays the XPS spectra acquired in the $\mathrm{Fe} 2 p$ and Ni $2 p$ regions. The analysis of the evolution of the $\mathrm{Fe} 2 p$ and $\mathrm{Ni} 2 p$ lineshapes upon room-temperature oxidation of $\mathrm{Ni} / \mathrm{Fe}(001)$ confirms the development of Fe oxides on the surface layer, but also reveals the presence on $\mathrm{Ni}^{2+}$ ions (see spectra B of Fig. 36). Spectra C of Fig. 36, acquired after annealing the film in UHV, reveal that the thermal treatment completely reduces the $\mathrm{Ni}$ ions, increasing the amount of oxidized Fe. The evolution of the lineshape in UPS spectra upon oxidation is shown in Fig. 37. It is possible to notice that the UPS spectra acquired after the oxidation of the $\mathrm{Ni} / \mathrm{Fe}(001)$ sample significantly differ from those of a $\mathrm{NiO} / \mathrm{Au}$ reference sample [282], confirming that the $\mathrm{Ni}$ oxidation is

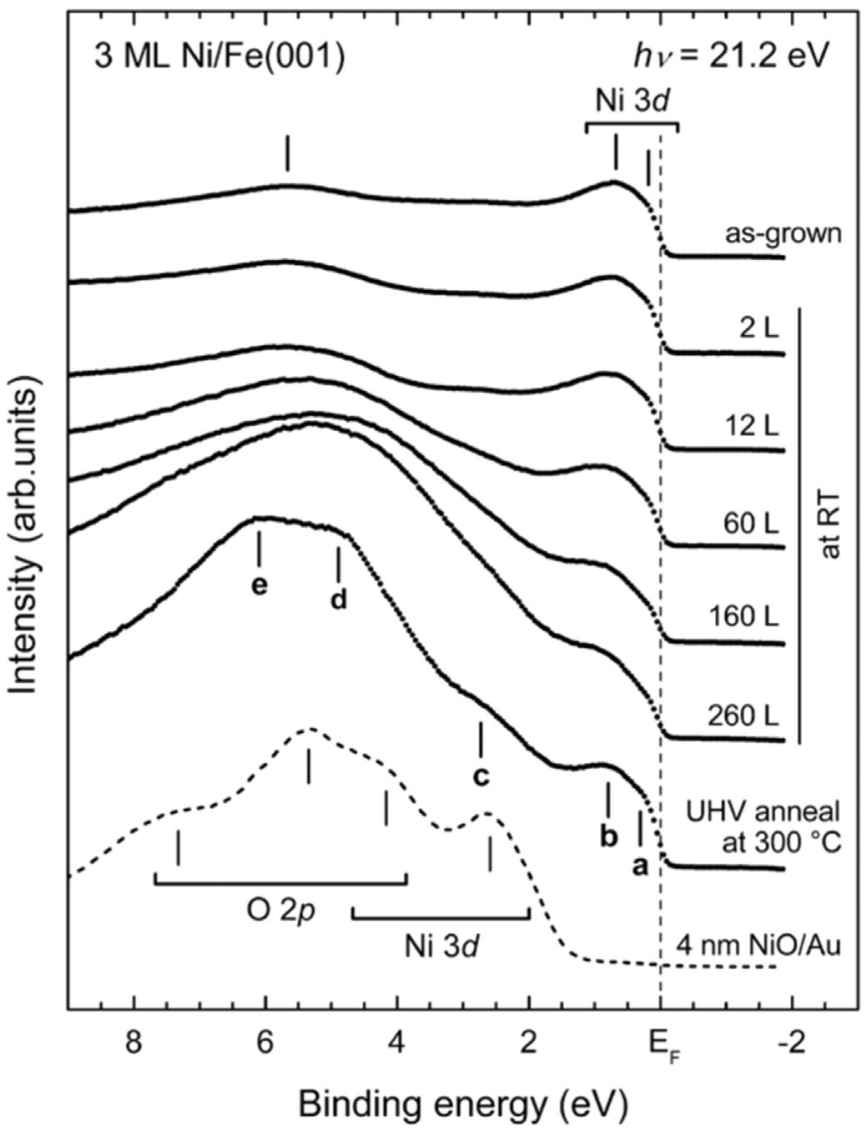

Fig. 37. UPS valence band of $3 \mathrm{ML} \mathrm{Ni/Fe(001).} \mathrm{Starting} \mathrm{from} \mathrm{the} \mathrm{top:} \mathrm{spectra}$ acquired on the as-grown sample, on the oxidized surface at increasing oxygen dose and after an annealing in UHV at $300^{\circ} \mathrm{C}$. Features at about $0.3 \mathrm{eV}$ (a), $0.8 \mathrm{eV}$ (b), $2.7 \mathrm{eV}$ (c), $4.9 \mathrm{eV}$ (d) and $6.0 \mathrm{eV}$ (e) are highlighted in the last spectrum. The spectrum acquired on the $\mathrm{NiO}$ reference sample is shown with a dashed line. Adapted with permission from Ref. [280]. Copyright (2014) by IOP Publishing.

negligible. Furthermore, the spectral features a-e visible after UHV annealing strongly resemble those observed in photoemission from the (111) face of $\mathrm{FeO}[283,284]$.

The presented results point towards the impossibility of obtaining an atomically sharp $\mathrm{NiO} / \mathrm{Fe}(001)$ interface. This conclusion can be rationalized from the one hand by considering the competition for oxidation between the $\mathrm{Ni}$ film and the more oxidizable $\mathrm{Fe}(001)$ substrate (see Table 1), on the other hand with the general oxidation reluctance of $\mathrm{Ni}$ films supported by a metallic substrate [285].

The observation of a non-reconstructed $\mathrm{FeO}(111)$ surface on top of the $\mathrm{Ni} / \mathrm{Fe}(001)$ square substrate deserves some further discussion for two reasons. In the first place, because of the quasi-hexagonal symmetry of the bcc (110) surface, $\mathrm{FeO}(111)$ like films generally form upon oxidation of the $\mathrm{Fe}(011)$ surface $[286,287]$ or by reactive growth of $\mathrm{Fe}$ oxide on $\mathrm{Fe}(011)$ [288]. Conversely, the formation of the $\mathrm{FeO}(111)$ film on the $\mathrm{Ni} / \mathrm{Fe}$ (001) substrate results in a symmetry-mismatched interface between the hexagonal oxide layer and the square substrate lattice. Generally, the stabilization of (111)-oriented rocksalt oxides on square substrates is associated with a large mismatch between the oxide overlayer and the substrate, as in the case of 

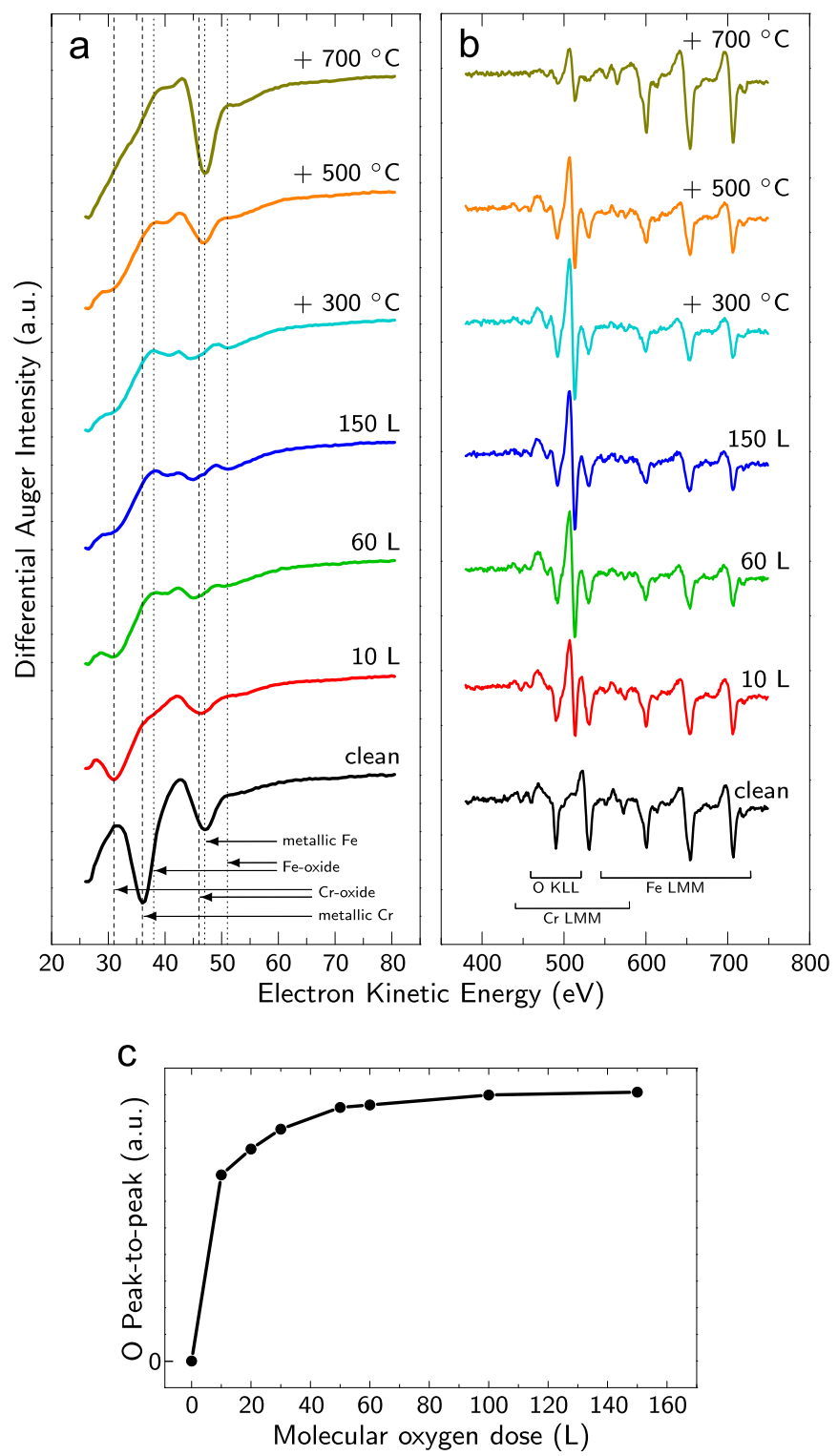

Fig. 38. (a) Low kinetic energy Auger spectra for a $\mathrm{Cr} / \mathrm{Fe}(001)$ sample at different oxygen exposures and after post-annealing treatments. The position of relevant metallic and oxide features for both $\mathrm{Cr}$ (dashed) and $\mathrm{Fe}$ (dotted) is indicated. (b) High kinetic energy Auger spectra corresponding to samples in (a), where the position of $\mathrm{Fe}$ and Cr LMM and O KLL transitions is highlighted. (c) Evolution of the oxygen peak-to-peak intensity as a function of the oxygen dose. Reprinted with permission from Ref. [279]. Copyright (2014) by Elsevier.

$\mathrm{MnO}$ on $\mathrm{Rh}(100)$ [289] or $\mathrm{CoO}$ on $\operatorname{Ir}(001)$ [290]. In addition, the (111) surfaces of bulk rocksalt oxides are unstable because of the development of a dipole moment perpendicular to the surface, ${ }^{2}$ due to the alternation of atomic planes formed by oppositely charged ions $[293,294]$. These two circumstances would conspire to rather induce the preferential stabilization of a $\mathrm{FeO}(001)$ surface, which is charge-compensated and

\footnotetext{
${ }^{2}$ It should be mentioned that only the (111) bulk termination of rocksalt oxides is rigorously unstable, while in the two-dimensional limit additional charge- and polarity-compensation mechanisms become operative, see for instance [291,292].
}

possesses a small lattice mismatch with respect to $\mathrm{Fe}(001)$. Further theoretical insights is therefore needed to rationalize the mechanisms driving the stabilization of the observed $\mathrm{FeO}$ (111)-like film.

A completely different oxidation path is observed in the case of $\mathrm{Fe}(001)$-supported $\mathrm{Cr}$ ultra-thin films [279,280]. In this case Fe segregation and oxidation is not observed during the early stages of oxygen exposure. Auger spectra acquired after oxidation and post-annealing treatments on $4 \mathrm{ML} \mathrm{Cr} / \mathrm{Fe}(001)$ samples are displayed in Fig. 38, where panels (a) and (b) refer to the low and high kinetic energy regions, respectively. In the oxygen-free sample, the peaks arising from metallic $\mathrm{Fe}(47 \mathrm{eV})$ and metallic $\mathrm{Cr}(36 \mathrm{eV})$ are visible [see Fig. 38(a)]. After exposure to $10 \mathrm{~L}$ of $\mathrm{O}_{2}$ and post-annealing treatment, the feature associated to metallic $\mathrm{Cr}$ is no longer visible, substituted by a peak at $31 \mathrm{eV}$, related to the presence of $\mathrm{Cr}$ oxide. Importantly, no features related to $\mathrm{Fe}$ oxide are present at this stage. Upon increasing the oxygen dose up to $150 \mathrm{~L}$, the characteristic shoulder of O-bound $\mathrm{Fe}$ appears in the spectrum. UHV annealing at increasing temperatures leads to the dissolution of the Cr oxide film, as visible in the upper spectra of Fig. 38(a) and (b). Notice that the oxygen uptake for the $\mathrm{Cr} / \mathrm{Fe}(001)$ system, displayed in Fig. 38(c), is steeper than that observed in the case of $\mathrm{Ni} / \mathrm{Fe}(001)$ oxidation [see Fig. 31(c)].

Despite $\mathrm{Cr}$ oxide overlayers can be stabilized on top of $\mathrm{Fe}(001)$, the resulting morphology is particularly rough, making their investigation at the atomic scale difficult. The acquired STM topography reveals that the surface roughness increases after the oxidation step [see Fig. 39(b) and (c) for $10 \mathrm{~L}$ and $150 \mathrm{~L}$ of oxygen exposure, respectively]. Annealing treatments up to $673 \mathrm{~K}$ do not significantly affect the sample morphology. On the other hand, annealing in UHV at $773 \mathrm{~K}$ induces the formation of holes in the $\mathrm{Cr}$ oxide film [Fig. 39(d)]. The STS data of Fig. 39(e) show that a conductivity gap is present on the remaining film, while the electronic structure of the holes is different and strongly resembles the one characteristic of $\mathrm{Fe}(001)-p(1 \times 1) \mathrm{O}$ (see Section 4.1).

The chemical stability of the $\mathrm{Cr}$ oxide/ $\mathrm{Fe}(001)$ interface is confirmed also by XPS. In this case, 3 ML-thick Cr films were grown using the $\mathrm{Fe}(001)-p(1 \times 1) \mathrm{O}$ surface as a substrate, i.e. the sample before oxidation (to which spectra A in Fig. 40 refer) already has a single layer of oxygen on top [278]. A room-temperature oxidation (spectra B of Fig. 40) partially oxidizes the $\mathrm{Cr}$ film, leaving however the $\mathrm{Fe}(001)$ substrate in a metallic state. The interface is stable even after annealing at $300{ }^{\circ} \mathrm{C}$ in UHV, as demonstrated by spectra $\mathrm{C}$ of Fig. 40 , where the Fe $2 p$ peaks do not present any feature related to $\mathrm{Fe}$ oxide species. On the other hand, the contribution from the metallic $\mathrm{Cr}$ layer is slightly attenuated, likely because of a partial interdiffusion of $\mathrm{Cr}$ atoms into the $\mathrm{Fe}(001)$ substrate bulk occurring after the annealing treatments. In order to completely convert the $\mathrm{Cr}$ film into $\mathrm{Cr}$ oxide, the oxidation must be performed at high temperatures. Spectra D of Fig. 40, acquired after further exposure to $10 \mathrm{~L}$ of $\mathrm{O}_{2}$ at $300{ }^{\circ} \mathrm{C}$, reveal that the $\mathrm{Cr}$ film is completely oxidized. The $\mathrm{Fe} 2 p$ lineshape displays a component characteristic of $\mathrm{Fe}^{2+}$ species, indicating 

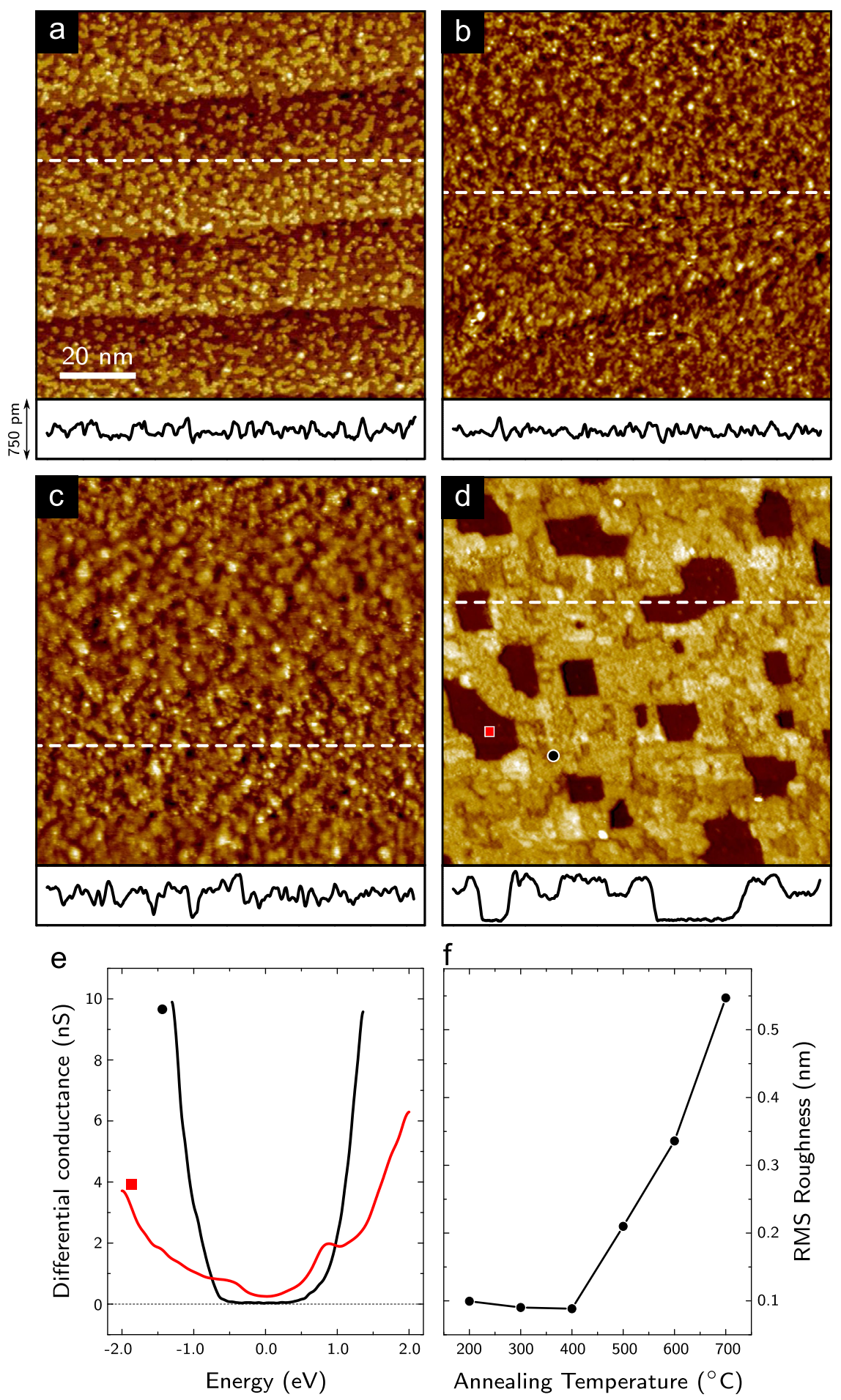

Fig. 39. STM topography of a $\mathrm{Cr} / \mathrm{Fe}(001)$ sample after oxidation and annealing. (a) As-grown $\mathrm{Cr}$ film, (b) 10 and (c) $150 \mathrm{~L}$ oxygen exposure, (d) $150 \mathrm{~L}+$ annealing at $773 \mathrm{~K}$. Height profiles along the corresponding dashed white lines are reported at the bottom of each panel. Vertical scale is $750 \mathrm{pm}$. (e) STS spectra acquired at correspondence with the holes (red square) and on the $\mathrm{Cr}$ film (black circle) in (d). (f) Root mean square (RMS) roughness of the sample measured on $200 \times 200 \mathrm{~nm}^{2}$ STM images as a function of the thermal treatments. Reprinted with permission from Ref. [279]. Copyright (2014) by Elsevier. (For interpretation of the references to color in this figure caption, the reader is referred to the web version of this paper.)

$\mathrm{Fe}(001)$ substrate oxidation. However, at variance with the $\mathrm{Ni} / \mathrm{Fe}(001)$ system, for the $\mathrm{Cr} / \mathrm{Fe}(001)-p(1 \times 1) \mathrm{O}$ sample $\mathrm{Fe}$ oxidation starts only after the complete oxidation of the $\mathrm{Cr}$ film, suggesting the feasibility of obtaining a sharp $\mathrm{Cr}$-oxide/Fe(001) interface by a proper choice of temperature and oxygen dosing. 


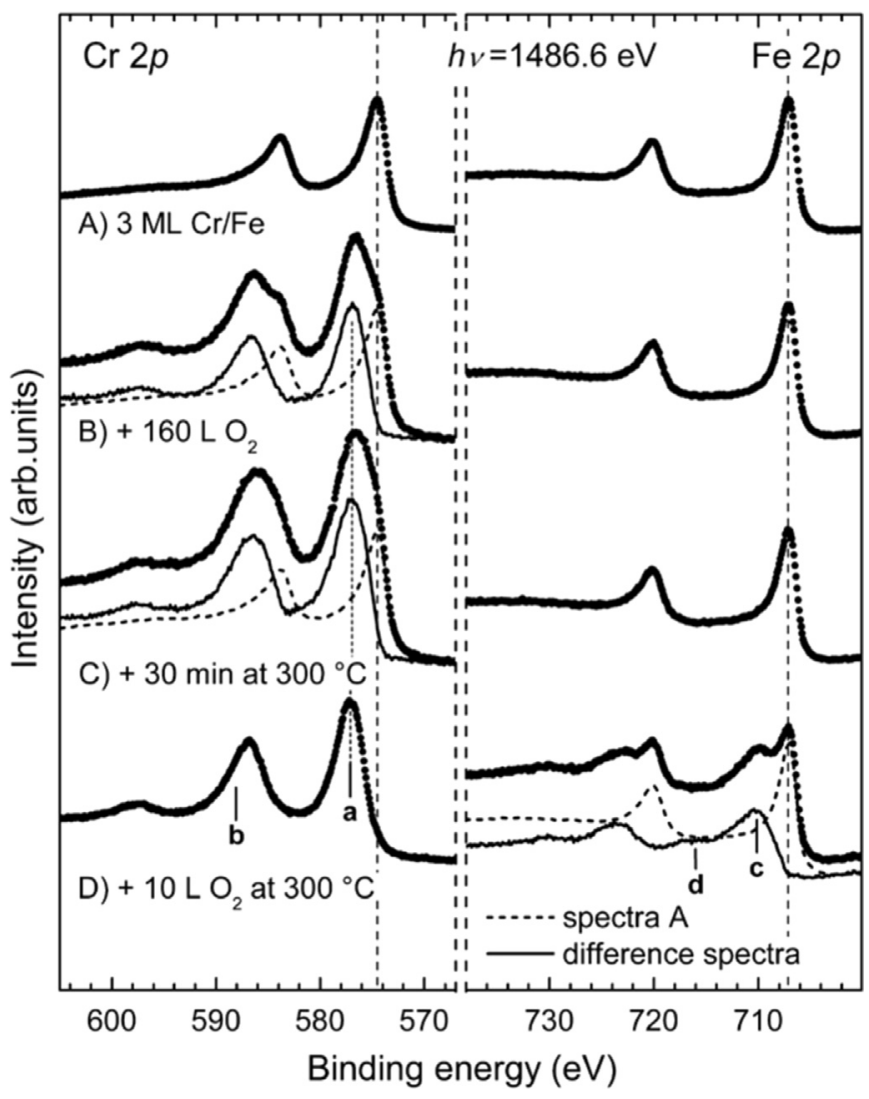

Fig. 40. XPS scans of the $\mathrm{Fe} 2 p$ and $\mathrm{Cr} 2 p$ regions of $3 \mathrm{ML} \mathrm{Cr} / \mathrm{Fe}(001)-p(1 \times 1)$ O. (A) As grown, (B) dosed with $160 \mathrm{~L}$ of $\mathrm{O}_{2}$ at room temperature, (C) annealed

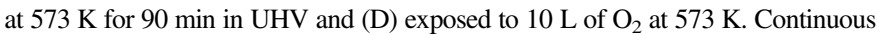
lines are difference spectra obtained by subtracting the A spectra (dashed lines in spectra from B to C) from the experimental data. Reprinted with permission from Ref. [280]. Copyright (2014) by IOP Publishing.

\section{Conclusions}

The presented experimental results reveal, on one hand, the extreme complexity of the metal-oxide interfaces, and on the other hand, the dramatic effects that even moderate compositional modifications of the interface can induce on the overall structural, electronic and magnetic properties of such heterostructures. The possibility to tune the properties of a material relies to a large extent on the experimental ability to control the structure and chemistry of the metal-oxide interface at the atomic level. Model systems as those presented here are fundamental for the rationalization of the processes occurring in layered $\mathrm{O} / \mathrm{M}$ and $\mathrm{M} / \mathrm{O}$ systems and allow for a direct correlation between their properties and interface composition. Such an approach has been and still is extensively applied on oxide ultra-thin films supported by inert metals, but can be also extended to investigate oxide films supported on highly reactive substrates, despite the additional experimental drawbacks encountered in the stabilization of well-ordered structures. In this respect, we have provided a survey of the information that can be obtained about the complex mechanisms driving the interface formation between a reactive metal and an oxide, with particular attention dedicated to the prototypical case of ultra-thin oxides grown of $\mathrm{Fe}(001)$. We are confident that, thanks to the extreme importance of metaloxide interfaces, their investigation will still be in the focus of fundamental research for the next years, providing important insights for future technological applications.

\section{Acknowledgments}

The authors would like to thank all the colleagues and friends, whom they had the opportunity to work and discuss with during their activity concerning this work. Among these, a special mention goes to (in alphabetical order) S. Achilli, G. Berti, C. E. Bottani, C. S. Casari, F. Donati, G. Fratesi, D. Giannotti, A. Li Bassi, M. Passoni, and M. I. Trioni.

This work was partially supported by the Italian Ministry of University and Research trough the FIRB project RBAP115AYN. Financial support was provided also by GEMINI (FP7-FET-OPEN X-TRACK No. 2013-0623). Computational resources were made available in part by CINECA (application code HP10C3YWUA). A. Picone would like to dedicate this work to Alice, who hopefully will live in a wonderland, who already made the world a wonderful place.

\section{References}

[1] H.-J. Freund, S. Shaikhutdinov, N. Nilius, Top. Catal. 57 (10-13) (2014) 822-832, http://dx.doi.org/10.1007/s11244-014-0276-6.

[2] X. Guo, Q. Fu, Y. Ning, M. Wei, M. Li, S. Zhang, Z. Jiang, X. Bao, J. Am. Chem. Soc. 134 (30) (2012) 12350-12353, http://dx.doi.org/10.1 021/ja3038883.

[3] S. Surnev, A. Fortunelli, F.P. Netzer, Chem. Rev. 113 (6) (2013) 4314-4372, http://dx.doi.org/10.1021/cr300307n URL 〈http://pubs.acs. org/doi/abs/10.1021/cr300307n $\rangle$.

[4] G. Pacchioni, L. Skuja, D.L. Griscom (Eds.), Defects in $\mathrm{SiO}_{2}$ and Related Dielectrics: Science and Technology, Kluwer, Dordrecht, 2000, 2012. http://dx.doi.org/10.1007/978-94-010-0944-7.

[5] C. Cagli, F. Nardi, B. Harteneck, Z. Tan, Y. Zhang, D. Ielmini, Small 7 (20) (2011) 2899-2905, http://dx.doi.org/10.1002/smll.201101157.

[6] V. Skumryev, S. Stoyanov, Y. Zhang, G. Hadjipanayis, D. Givord, J. Nogués, Nature 423 (2003) 850-853, http://dx.doi.org/10.1038/nature 01687 URL 〈http://www.nature.com/nature/journal/v423/n6942/full/nat ure01687.html $>$.

[7] S. Valeri, G. Pacchioni (Eds.), Oxide Ultrathin Films, Wiley-VCH Verlag GmbH \& Co. KGaA, Weinheim, 2011 Chapter 6, URL 〈http://eu.wiley. com/WileyCDA/WileyTitle/productCd-352733016X.html $>$.

[8] R. Gupta, N. Birbilis, Corros. Sci. 92 (2015) 1-15, http://dx.doi.org/10.101 6/j.corsci.2014.11.041 URL 〈http://www.sciencedirect.com/science/article/ pii/S0010938X14005629〉.

[9] A. Stoneham, P. Tasker, J. Phys. 49 (C-5) (1988) 99-113, http://dx.doi.org/ 10.1051/jphyscol:1988508.

[10] F.H. Stott, Rep. Prog. Phys. 50 (7) (1987) 861 URL /http://stacks.iop.org/ $0034-4885 / 50 / \mathrm{i}=7 / \mathrm{a}=002\rangle$.

[11] J. Wu, D. Carlton, J.S. Park, Y. Meng, E. Arenholz, A. Doran, A. T. Young, A. Scholl, C. Hwang, H.W. Zhao, J. Bokor, Z.Q. Qiu, Nat. Phys. 7 (4) (2011) 303, http://dx.doi.org/10.1038/NPHYS1891.

[12] M. Finazzi, L. Duò, F. Ciccacci, Surf. Sci. Rep. 64 (4) (2009) 139-167 http://dx.doi.org/10.1016/j.surfrep.2008.12.003.

[13] G. Pacchioni, H. Freund, Chem. Rev. 113 (6) (2013) 4035-4072 http://dx.doi.org/10.1021/cr3002017.

[14] G. Radaelli, D. Petti, M. Cantoni, C. Rinaldi, R. Bertacco, J. Appl. Phys. 115 (17), (2014). http://dx.doi.org/10.1063/1.4870915, URL <http://scita tion.aip.org/content/aip/journal/jap/115/17/10.1063/1.4870915〉. 
[15] J. Mathon, A. Umerski, Phys. Rev. B 63 (2001) 220403, http:// dx.doi.org/ 10.1103/PhysRevB.63.220403 URL 〈http://link.aps.org/ doi/10.1103/Phys RevB.63.220403〉.

[16] S. Yuasa, T. Nagahama, A. Fukushima, Y. Suzuki, K. Ando, Nat. Mater. 3 (2004) 868-871, http://dx.doi.org/10.1038/nmat1257.

[17] S.A. Chambers, Surf. Sci. Rep. 39 (5-6) (2000) 105-180, http:// dx.doi.org/ 10.1016/S0167-5729(00)00005-4 URL 〈http:// www.sciencedirect.com/science/article/pii/S0167572900000054).

[18] C.T. Campbell, Surf. Sci. Rep. 27 (1-3) (1997) 1-111, http:// dx.doi.org/10.1016/S0167-5729(96)00011-8 URL 〈http:// www.sciencedirect.com/science/article/pii/S0167572996000118>.

[19] Q. Fu, T. Wagner, Surf. Sci. Rep. 62 (11) (2007) 431-498 http://dx.doi.org/10.1016/j.surfrep.2007.07.001 URL 〈http://www.science direct.com/science/article/pii/S0167572907000635〉.

[20] S. Schintke, S. Messerli, M. Pivetta, F. Patthey, L. Libioulle, M. Stengel, A. De Vita, W.-D. Schneider, Phys. Rev. Lett. 87 (2001) 276801 http://dx.doi.org/10.1103/PhysRevLett.87.276801 URL 〈http://link.aps. org/doi/10.1103/PhysRevLett.87.276801>.

[21] S. Schintke, W.-D. Schneider, J. Phys.: Condens. Matter 16 (4) (2004) R49 URL 〈http://stacks.iop.org/0953-8984/16/i=4/a=R02〉.

[22] D.R. Lide (Ed.), CRC Handbook of Chemistry and Physics, CRC Press, Boca Raton, 2010.

[23] J.A. Dean (Ed.), Lange's Handbook of Chemistry, McGraw-Hill, Inc., Knoxville, 1998.

[24] G. Pacchioni, Phys. Chem. Chem. Phys. 15 (2013) 1737-1757 http://dx.doi.org/10.1039/C2CP43731G.

[25] M.T. Greiner, L. Chai, M.G. Helander, W.-M. Tang, Z.-H. Lu, Adv. Funct. Mater. 23 (2) (2013) 215-226, http://dx.doi.org/10.1002/ adfm.201200993.

[26] A. Calloni, A. Ferrari, A. Brambilla, F. Ciccacci, L. Duò, Thin Solid Films 520 (11) (2012) 3922-3926, http://dx.doi.org/10.1016/ j.tsf.2012.01.045 URL 〈http://www.sciencedirect.com/science/article/pii/ S004060901 2000831>.

[27] Z. Li, Z. Zhang, Y.K. Kim, R.S. Smith, F. Netzer, B.D. Kay, R. Rousseau, Z. Dohnálek, J. Phys. Chem. C 115 (13) (2011) 5773-5783, http://dx.doi.org/10.1021/jp1108976.

[28] R. de Masi, D. Reinicke, F. Müller, P. Steiner, S. Hüfner, Surf. Sci. 515 (2-3) (2002) 523-537, http://dx.doi.org/10.1016/S0039-6028(02)019 70-2 URL 〈http://www.sciencedirect.com/science/article/pii/S0039602 802019702).

[29] S. Benedetti, P. Torelli, P. Luches, A. Rota, S. Valeri, Surf. Sci. 600 (18) (2006) 4251-4255, http://dx.doi.org/10.1016/j.susc.2006.01.152 URL 〈http://www.sciencedirect.com/science/article/pii/S0039602806005577〉.

[30] P. Luches, F. Pagliuca, S. Valeri, F. Illas, G. Preda, G. Pacchioni, J. Phys. Chem. C 116 (1) (2012) 1122-1132, http://dx.doi.org/10.1021/ jp210241c.

[31] J. Schoiswohl, M. Sock, S. Eck, S. Surnev, M.G. Ramsey, F.P. Netzer, G. Kresse, Phys. Rev. B 69 (2004) 155403, http://dx.doi.org/ 10.1103/PhysRevB.69.155403 URL 〈http://link.aps.org/doi/10.1103/Phys RevB.69.155403〉.

[32] J. Schoiswohl, S. Surnev, M. Sock, S. Eck, M.G. Ramsey, F.P. Netzer, G. Kresse, Phys. Rev. B 71 (2005) 165437, http://dx.doi.org/ 10.1103/PhysRevB.71.165437 URL 〈http://link.aps.org/doi/10.1103/Phys RevB.71.165437).

[33] A.S. Walton, J. Fester, M. Bajdich, M.A. Arman, J. Osiecki, J. Knudsen, A. Vojvodic, J.V. Lauritsen, ACS Nano 9 (3) (2015) 2445-2453, http://dx.doi.org/10.1021/acsnano.5b00158.

[34] T.J. Regan, H. Ohldag, C. Stamm, F. Nolting, J. Lüning, J. Stöhr, R.L. White, Phys. Rev. B 64 (2001) 214422, http://dx.doi.org/10.1103/ PhysRev B.64.214422 URL 〈http://link.aps.org/doi/10.1103/ PhysRevB.64.214422〉.

[35] S. Benedetti, P. Luches, M. Liberati, S. Valeri, Surf. Sci. 572 (23) (2004) L348-L354, http://dx.doi.org/10.1016/j.susc.2004.09.025 URL 〈http:// www.sciencedirect.com/science/article/pii/S0039602804012440〉.

[36] P. Luches, M. Liberati, S. Valeri, Surf. Sci. 532-535 (0) (2003) 409-414, http://dx.doi.org/10.1016/S0039-6028(03)00119-5 URL 〈http://www.sciencedirect.com/science/article/pii/S0039602803001195〉.

[37] P. Luches, V. Bellini, S. Colonna, L. Di Giustino, F. Manghi, S. Valeri, F. Boscherini, Phys. Rev. Lett. 96 (2006) 106106, http://dx.doi.org/
10.1103/PhysRevLett.96.106106 URL 〈http://link.aps.org/ doi/10.1103/PhysRevLett.96.106106 .

[38] N. Srivastava, P.C. Srivastava, J. Appl. Phys. 111 (12) (2012) 123909, http://dx.doi.org/10.1063/1.4729857 URL 〈http://scitation.aip. org/content/aip/journal/jap/111/12/10.1063/1.4729857〉.

[39] N. Srivastava, T. Shripathi, P. Srivastava, J. Electron Spectrosc. Relat. Phenom. 191 (0) (2013) 20-26, http://dx.doi.org/10.1016/ j.elspec.2013.09.003 URL 〈http://www.sciencedirect.com/science/arti cle/pii/S0368204813001461〉.

[40] V. Bellini, L. Di Giustino, F. Manghi, Phys. Rev. B 76 (2007) 214432, http://dx.doi.org/10.1103/PhysRevB.76.214432 URL 〈http:// link.aps.org/doi/10.1103/PhysRevB.76.214432〉.

[41] M. Finazzi, A. Brambilla, L. Duò, G. Ghiringhelli, M. Portalupi, F. Ciccacci, M. Zacchigna, M. Zangrando, Phys. Rev. B 70 (2004) 235420, http://dx.doi.org/10.1103/PhysRevB.70.235420 URL /http://link. aps.org/doi/10.1103/PhysRevB.70.235420〉.

[42] L. Duò, M. Portalupi, M. Marcon, R. Bertacco, F. Ciccacci, Surf. Sci. 518 (3)(2002) 234-242, http://dx.doi.org/10.1016/S0039-6028(02)02194-5 URL 〈http://www.sciencedirect.com/science/article/pii/S0039602802021945〉.

[43] E. Młyńczak, P. Luches, S. Valeri, J. Korecki, J. Appl. Phys. 113 (23) (2013) 234315, http://dx.doi.org/10.1063/1.4811528 URL 〈http:// scitation.aip.org/content/aip/journal/jap/113/23/10.1063/1.4811528〉.

[44] R. Abrudan, J. Miguel, M. Bernien, C. Tieg, M. Piantek, J. Kirschner, W. Kuch, Phys. Rev. B 77 (2008) 014411, http://dx.doi.org/10.1103/ PhysRevB.77.014411 URL 〈http://link.aps.org/doi/10.1103/PhysRevB. $77.014411\rangle$.

[45] E. Młyńczak, B. Matlak, A. Kozioł-Rachwał, J. Gurgul, N. Spiridis, J. Korecki, Phys. Rev. B 88 (2013) 085442, http://dx.doi.org/ 10.1103/PhysRevB.88.085442 URL 〈http://link.aps.org/doi/10.1103/Phys RevB.88.085442>.

[46] A. Brambilla, P. Sessi, M. Cantoni, L. Duò, M. Finazzi, F. Ciccacci, Thin Solid Films 516 (21) (2008) 7519-7524, http://dx.doi.org/10.1016/ j.tsf.2008.04.058 URL 〈http://www.sciencedirect.com/science/article/pii/ S0040609008004525〉.

[47] R. Bali, M.M. Soares, A.Y. Ramos, H.C.N. Tolentino, F. Yildiz, C. Boudot, O. Proux, M. De Santis, M. Przybylski, J. Kirschner, Appl. Phys. Lett. 100 (13) (2012) 132403, http://dx.doi.org/10.1063/ 1.3695158 URL 〈http://scitation.aip.org/content/aip/journal/apl/100/13/ $10.1063 / 1.3695158\rangle$.

[48] A. Brambilla, A. Calloni, G. Berti, G. Bussetti, L. Duò, F. Ciccacci, J. Phys. Chem. C 117 (18) (2013) 9229-9236, http://dx.doi.org/ 10.1021/jp400159j.

[49] U. Diebold, Surf. Sci. Rep. 48 (58) (2003) 53-229, http://dx.doi.org/ 10.1016/S0167-5729(02)00100-0 URL 〈http://www.sciencedirect.com/ science/article/pii/S0167572902001000

[50] P. Luches, S. Benedetti, M. Liberati, F. Boscherini, I. Pronin, S. Valeri, Surf. Sci. 583 (23) (2005) 191-198, http://dx.doi.org/10.1016/ j.susc.2005.03.038 URL 〈http://www.sciencedirect.com/science/article/ pii/S0039602805003171〉.

[51] G. Sharma, A. Gupta, P. Rajput, Rüffer, J. Phys. D: Appl. Phys. 47 (34) (2014) 345305 URL $\langle$ http://stacks.iop.org/0022-3727/47/i=34/a=345305 $\rangle$.

[52] J. Balogh, I. Dézsi, C. Fetzer, J. Korecki, A. Kozioł-Rachwał, E. Młyńczak, A. Nakanishi, Phys. Rev. B 87 (2013) 174415, http://dx.doi.org/ 10.1103/PhysRevB.87.174415 URL 〈http://link.aps.org/doi/10.1103/Phys RevB.87.174415).

[53] S.R. Spurgeon, J.D. Sloppy, R. Tao, R.F. Klie, S.E. Lofland, J. K. Baldwin, A. Misra, M.L. Taheri, J. Appl. Phys. 112 (1) (2012) 013905, http://dx.doi.org/10.1063/1.4730630 URL 〈http://scitation.aip. org/content/aip/journal/jap/112/1/10.1063/1.4730630〉.

[54] E. Mlyńczak, K. Freindl, N. Spiridis, J. Korecki, J. Appl. Phys. 113 (2) (2013) 024320, http://dx.doi.org/10.1063/1.4775707 URL 〈http://scitation.aip.org/con tent/aip/journal/jap/113/2/10.1063/1.4775707>.

[55] A. Cattoni, D. Petti, S. Brivio, M. Cantoni, R. Bertacco, F. Ciccacci, Phys. Rev. B 80 (2009) 104437, http://dx.doi.org/10.1103/Phys RevB.80.104437 URL 〈http://link.aps.org/doi/10.1103/PhysRevB.80. $104437\rangle$ 
[56] L. Plucinski, Y. Zhao, B. Sinkovic, E. Vescovo, Phys. Rev. B 75 (2007) 214411, http://dx.doi.org/10.1103/PhysRevB.75.214411 URL 〈http://link. aps.org/doi/10.1103/PhysRevB.75.214411 .

[57] A. Tekiel, S. Fostner, J. Topple, Y. Miyahara, P. Grütter, Appl. Surf. Sci. 273 (2013) 247-252, http://dx.doi.org/10.1016/ j.apsusc.2013.02.024 URL 〈http://www.sciencedirect.com/science/article/pii/ S0169433213003322>.

[58] H.L. Meyerheim, R. Popescu, N. Jedrecy, M. Vedpathak, M. SauvageSimkin, R. Pinchaux, B. Heinrich, J. Kirschner, Phys. Rev. B 65 (2002) 144433, http://dx.doi.org/10.1103/PhysRevB.65.144433 URL 〈http://link. aps.org/doi/10.1103/PhysRevB.65.144433〉.

[59] T. Bertrams, H. Neddermeyer, J. Vac. Sci. Technol. B 14 (2) (1996) 1141-1144, http://dx.doi.org/10.1116/1.588416 URL 〈http://scitation.aip. org/content/avs/journal/jvstb/14/2/10.1116/1.588416).

[60] I. Sebastian, T. Bertrams, K. Meinel, H. Neddermeyer, Faraday Discuss. 114 (1999) 129-140, http://dx.doi.org/10.1039/A903416A.

[61] M. Caffio, A. Atrei, B. Cortigiani, G. Rovida, J. Phys.: Condens. Matter 18 (8) (2006) 2379 URL 〈http://stacks.iop.org/0953-8984/18/i=8/a=003〉.

[62] A. Dhaka, D. Sander, H.L. Meyerheim, K. Mohseni, E. Soyka, J. Kirschner, W.A. Adeagbo, G. Fischer, A. Ernst, W. Hergert, Phys. Rev. B 84 (2011) 195441, http://dx.doi.org/10.1103/PhysRevB.84. 195441 URL 〈http://link.aps.org/doi/10.1103/PhysRevB.84.195441〉.

[63] W. Steurer, F. Allegretti, S. Surnev, G. Barcaro, L. Sementa, F. Negreiros, A. Fortunelli, F.P. Netzer, Phys. Rev. B 84 (2011) 115446, http://dx.doi.org/10.1103/PhysRevB.84.115446 URL 〈http:// link.aps.org/doi/10.1103/PhysRevB.84.115446〉.

[64] W. Steurer, S. Surnev, A. Fortunelli, F.P. Netzer, Surf. Sci. 606 (910) (2012) 803-807, http://dx.doi.org/10.1016/j.susc.2012.01.012 URL 〈http://www. sciencedirect.com/science/article/pii/S0039602812000283〉.

[65] S.H. Phark, Y.J. Chang, T.W. Noh, J.-S. Kim, Phys. Rev. B 80 (2009) 035426, http://dx.doi.org/10.1103/PhysRevB.80.035426 URL /http://link. aps.org/doi/10.1103/PhysRevB.80.035426).

[66] A. Ouvrard, J. Niebauer, A. Ghalgaoui, C. Barth, C.R. Henry, B. Bourguignon, J. Phys. Chem. C 115 (16) (2011) 8034-8041, http://dx.doi.org/10.1021/jp1095823.

[67] S. Baumann, I.G. Rau, S. Loth, C.P. Lutz, A.J. Heinrich, ACS Nano 8 (2) (2014) 1739-1744, http://dx.doi.org/10.1021/nn4061034.

[68] L.R. Merte, M. Shipilin, S. Ataran, S. Blomberg, C. Zhang, A. Mikkelsen, J. Gustafson, E. Lundgren, J. Phys. Chem. C 119 (5) (2015) 2572-2582, http://dx.doi.org/10.1021/jp511496w.

[69] N.A. Khan, C. Matranga, Surf. Sci. 602 (4) (2008) 932-942, http://dx.doi.org/10.1016/j.susc.2007.12.027 URL 〈http://www.sciencedir ect.com/science/article/pii/S0039602807012216〉.

[70] M. Li, E.I. Altman, J. Phys. Chem. C 118 (24) (2014) 12706-12716, http://dx.doi.org/10.1021/jp411375w.

[71] L.-Y. Ma, A. Picone, M. Wagner, S. Surnev, G. Barcaro, A. Fortunelli, F.P. Netzer, J. Phys. Chem. C 117 (36) (2013) 18464-18474, http://dx.doi.org/10.1021/jp4052424.

[72] M. Sambi, R. Sensolo, G. Rizzi, M. Petukhov, G. Granozzi, Surf. Sci. 537 (13) (2003) 36-54, http://dx.doi.org/10.1016/ S0039-6028(03)00641-1 URL 〈http://www.sciencedirect.com/science/article/ pii/S0039602803006411〉.

[73] F. Li, F. Allegretti, S. Surnev, F. Netzer, Y. Zhang, W.-B. Zhang, K. Reuter, Surf. Sci. 604 (1920) (2010) 1813-1819, http://dx.doi.org/10.1016/j.susc.2010.07.012 URL $\langle$ http://www.sciencedir ect.com/science/article/pii/S0039602810002980〉.

[74] B. Herd, M. Knapp, H. Over, J. Phys. Chem. C 116 (46) (2012) 24649-24660, http://dx.doi.org/10.1021/jp3085155.

[75] J. Gustafson, A. Resta, A. Mikkelsen, R. Westerström, J.N. Andersen, E. Lundgren, J. Weissenrieder, M. Schmid, P. Varga, N. Kasper, X. Torrelles, S. Ferrer, F. Mittendorfer, G. Kresse, Phys. Rev. B 74 (2006) 035401, http://dx.doi.org/10.1103/PhysRevB.74.035401 URL 〈http://link. aps.org/doi/10.1103/PhysRevB.74.035401>.

[76] F. Li, F. Allegretti, S. Surnev, F.P. Netzer, Surf. Sci. 604 (1718) (2010) L43-L47, http://dx.doi.org/10.1016/j.susc.2010.05.017 URL 〈http:// www.sciencedirect.com/science/article/pii/S0039602810002190〉.

[77] C. Franchini, F. Li, S. Surnev, R. Podloucky, F. Allegretti, F.P. Netzer, J. Phys.: Condens. Matter 24 (4) (2012) 042001 URL 〈http:// stacks.iop.org $/ 0953-8984 / 24 / \mathrm{i}=4 / \mathrm{a}=042001\rangle$.
[78] J. Pal, M. Smerieri, E. Celasco, L. Savio, L. Vattuone, M. Rocca, Phys. Rev. Lett. 112 (2014) 126102, http://dx.doi.org/10.1103/ PhysRevLett.112.126102 URL 〈http://link.aps.org/doi/10.1103/PhysRev Lett.112.126102>.

[79] J. Pal, M. Smerieri, E. Celasco, L. Savio, L. Vattuone, R. Ferrando, S. Tosoni, L. Giordano, G. Pacchioni, M. Rocca, J. Phys. Chem. C 118 (45) (2014) 26091-26102, http://dx.doi.org/10.1021/jp507718n URL 〈http://dx.doi.org/10. 1021/jp507718n $>$

[80] I. Palacio, M. Monti, J.F. Marco, K.F. McCarty, J. de la Figuera, J. Phys.: Condens. Matter 25 (48) (2013) 484001 URL 〈http:// stacks.iop.org $/ 0953-8984 / 25 / \mathrm{i}=48 / \mathrm{a}=484001\rangle$.

[81] L. Vitos, A. Ruban, H. Skriver, J. Kollar, Surf. Sci. 411 (12) (1998) 186-202, http://dx.doi.org/10.1016/S0039-6028(98)00363-X URL $\langle$ http://www.sciencedirect.com/science/article/pii/S003960289800363X〉.

[82] B.S. Youmbi, F. Calvayrac, Surf. Sci. 621 (2014) 1-6 http://dx.doi.org/10.1016/j.susc.2013.10.012 URL 〈http://www.sciencedir ect.com/science/article/pii/S0039602813003087〉.

[83] D. Santos-Carballal, A. Roldan, R. Grau-Crespo, N.H. de Leeuw, Phys. Chem. Chem. Phys. 16 (2014) 21082-21097, http://dx.doi.org/10.1039/C4CP00529E.

[84] S.R. Lu, R. Yu, J. Zhu, Phys. Rev. B 87 (2013) 165436 http://dx.doi.org/10.1103/PhysRevB.87.165436 URL 〈http://link.aps.org/ doi/10.1103/PhysRevB.87.165436).

[85] D. Wolf, Phys. Rev. Lett. 68 (1992) 33153318, http://dx.doi.org/10.1103/PhysRevLett.68.3315 URL 〈http:// link.aps.org/doi/ 10.1103/PhysRevLett.68.3315 .

[86] W. Mackrodt, Phys. Chem. Miner. 15 (3) (1988) 228-237, http://dx.doi.org/10.1007/BF00307511.

[87] C. Rehbein, N.M. Harrison, A. Wander, Phys. Rev. B 54 (1996) 14066-14070, http://dx.doi.org/10.1103/PhysRevB.54.14066 URL 〈http://link.aps.org/doi/10.1103/PhysRevB.54.14066〉.

[88] S.A. Chambers, T. Droubay, D.R. Jennison, T.R. Mattsson, Science 297 (5582) (2002) 827-831, http://dx.doi.org/10.1126/science.1073404 URL 〈http://www.sciencemag.org/content/297/5582/827.abstract〉.

[89] J.F. Sanz, N.C. Hernández, Phys. Rev. Lett. 94 (2005) 016104, http://dx.doi.org/10.1103/PhysRevLett.94.016104 URL 〈http:// link.aps.org/doi/10.1103/PhysRevLett.94.016104〉.

[90] Q. Fu, T. Wagner, Surf. Sci 505 (2002) 39-48, http:// dx.doi.org/10.1016/S0039-6028(02)01392-4 URL 〈http:// www.sciencedirect.com/science/article/pii/S0039602802013924〉.

[91] X. Shao, N. Nilius, H.-J. Freund, Phys. Rev. B 85 (2012) 115444, http://dx.doi.org/10.1103/PhysRevB.85.115444 URL 〈http:// link.aps.org/doi/10.1103/PhysRevB.85.115444).

[92] D. Ricci, A. Bongiorno, G. Pacchioni, U. Landman, Phys. Rev. Lett. 97 (2006) 036106, http://dx.doi.org/10.1103/PhysRevLett.97.036106 URL 〈http://link. aps.org/doi/10.1103/PhysRevLett.97.036106〉.

[93] M. Sterrer, T. Risse, M. Heyde, H.-P. Rust, H.-J. Freund, Phys. Rev. Lett. 98 (2007) 206103, http://dx.doi.org/10.1103/PhysRevLett.98.206103 URL 〈http://link.aps.org/doi/10.1103/PhysRevLett.98.206103〉.

[94] W. Jauch, M. Reehuis, H.J. Bleif, F. Kubanek, P. Pattison, Phys. Rev. B 64 (2001) 052102, http://dx.doi.org/10.1103/PhysRevB.64.052102.

[95] W.L. Smith, A.D. Hobson, Acta Crystallogr. Sect. B 29 (2) (1973) 362-363, http://dx.doi.org/10.1107/S0567740873002505 URL 〈http://dx. doi.org/10.1107/S0567740873002505〉.

[96] L.C. Bartel, B. Morosin, Phys. Rev. B 3 (1971) 1039-1043, http://dx.doi.org/10.1103/PhysRevB.3.1039.

[97] P. Kaercher, S. Speziale, L. Miyagi, W. Kanitpanyacharoen, H.-R. Wenk, Phys. Chem. Miner. 39 (8) (2012) 613-626, http:// dx.doi.org/ 10.1007/s00269-012-0516-x.

[98] E. Nazarenko, J.E. Lorenzo, Y. Joly, J.L. Hodeau, D. Mannix, C. Marin, Phys. Rev. Lett. 97 (2006) 056403, http://dx.doi.org/10.1103/PhysRevLett. 97.056403.

[99] K. Doll, M. Dolg, H. Stoll, Phys. Rev. B 54 (1996) 13529-13535 http://dx.doi.org/10.1103/PhysRevB.54.13529 URL〈http://link.aps.org/ doi/10.1103/PhysRevB.54.13529).

[100] F.W. Lytle, J. Appl. Phys. 35 (7) (1964) 22122215, http://dx.doi.org/10.1063/1.1702820. 
[101] G. Renaud, P. Guénard, A. Barbier, Phys. Rev. B 58 (1998) 7310-7318, http://dx.doi.org/10.1103/PhysRevB.58.7310 URL /http:// link.aps.org/doi/10.1103/PhysRevB.58.7310〉.

[102] P. Torelli, E. Soares, G. Renaud, S. Valeri, X. Guo, P. Luches, Surf. Sci. 601 (13) (2007) 2651-2655, http://dx.doi.org/10.1016/j.susc.2006.11.063 URL 〈http://www.sciencedirect.com/science/article/pii/S0039602806012532〉.

[103] S. Benedetti, P. Torelli, S. Valeri, H.M. Benia, N. Nilius, G. Renaud, Phys. Rev. B 78 (2008) 195411, http://dx.doi.org/10.1103/PhysRevB. 78.195411 URL 〈http://link.aps.org/doi/10.1103/PhysRevB.78.195411〉.

[104] F.P. Netzer, Surf. Rev. Lett. 09 (03n04) (2002) 1553-1563 http://

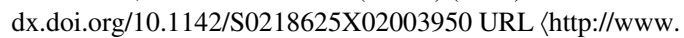
worldscientific.com/doi/abs/10.1142/S0218625X02003950〉.

[105] S. Agnoli, M. Sambi, G. Granozzi, A. Atrei, M. Caffio, G. Rovida, Surf. Sci. 576 (13) (2005) 1-8, http://dx.doi.org/10.1016/j.susc.2004.11.032 URL 〈http://www.sciencedirect.com/science/article/pii/S003960280401516X).

[106] C. Franchini, R. Podloucky, F. Allegretti, F. Li, G. Parteder, S. Surnev, F.P. Netzer, Phys. Rev. B 79 (2009) 035420, http://dx.doi.org/10.1103/PhysRevB.79.035420 URL 〈http://link.aps. org/doi/10.1103/PhysRevB.79.035420 .

[107] F. Allegretti, G. Parteder, L. Gragnaniello, S. Surnev, F. Netzer, A. Barolo, S. Agnoli, G. Granozzi, C. Franchini, R. Podloucky, Surf. Sci. 604 (56) (2010) 529-534, http://dx.doi.org/10.1016/j.susc.2009.12.018 URL 〈http://www.sciencedirect.com/science/article/pii/S003960280900795X〉.

[108] X. Shao, P. Myrach, N. Nilius, H.-J. Freund, J. Phys. Chem. C 115 (17) (2011) 8784-8789, http://dx.doi.org/10.1021/jp201852x.

[109] X. Shao, N. Nilius, P. Myrach, H.-J. Freund, U. Martinez, S. Prada, L. Giordano, G. Pacchioni, Phys. Rev. B 83 (2011) 245407, http://dx.doi.org/10.1103/PhysRevB.83.245407 URL 〈http://link.aps. org/doi/10.1103/PhysRevB.83.245407).

[110] S. Shaikhutdinov, M. Ritter, W. Weiss, Phys. Rev. B 62 (2000) 7535-7541, http://dx.doi.org/10.1103/PhysRevB.62.7535 URL 〈http:// link.aps.org/doi/10.1103/PhysRevB.62.7535〉.

[111] D. Bruns, I. Kiesel, S. Jentsch, S. Lindemann, C. Otte, T. Schemme, T. Kuschel, J. Wollschlger, J. Phys.: Condens. Matter 26 (31) (2014) 315001 URL 〈http://stacks.iop.org/0953-8984/26/i=31/a =315001〉. K.

[112] Biedermann, M. Gubo, L. Hammer, K. Heinz, J. Phys.: Condens. Matter 21 (18) (2009) 185003 URL 〈http://stacks.iop.org/0953-8984/21/i=18/a= $185003\rangle$.

[113] C. Tröppner, T. Schmitt, M. Reuschl, L. Hammer, M.A. Schneider, F. Mittendorfer, J. Redinger, R. Podloucky, M. Weinert, Phys. Rev. B 86 (2012) 235407, http://dx.doi.org/10.1103/PhysRevB.86.235407 URL 〈http://link.aps.org/doi/10.1103/PhysRevB.86.235407〉.

[114] F. Li, G. Parteder, F. Allegretti, C. Franchini, R. Podloucky, S. Surnev, F.P. Netzer, J. Phys.: Condens. Matter 21 (13) (2009) 134008 URL $\langle$ http://stacks.iop.org/0953-8984/21/i=13/a = 134008〉. Y.

[115] Pan, S. Benedetti, N. Nilius, H.-J. Freund, Phys. Rev. B 84 (2011) 075456, http://dx.doi.org/10.1103/PhysRevB.84.075456 URL 〈http:// link.aps.org/doi/10.1103/PhysRevB.84.075456〉.

[116] M. Gubo, C. Ebensperger, W. Meyer, L. Hammer, K. Heinz, F. Mittendorfer, J. Redinger, Phys. Rev. Lett. 108 (2012) 066101, http://dx.doi.org/10.1103/PhysRevLett.108.066101 URL 〈http://link.aps. org/doi/10.1103/PhysRevLett.108.066101>.

[117] E.M. Davis, K. Zhang, Y. Cui, H. Kuhlenbeck, S. Shaikhutdinov, H.J. Freund, Surf. Sci. 636 (2015) 42-46, http://dx.doi.org/10.1016/ j.susc.2015.02.004 URL 〈http://www.sciencedirect.com/science/ article/pii/S0039602815000394〉.

[118] N. Spiridis, J. Barbasz, Z. Łodziana, J. Korecki, Phys. Rev. B 74 (2006) 155423, http://dx.doi.org/10.1103/PhysRevB.74.155423 URL 〈http:// link.aps.org/doi/10.1103/PhysRevB.74.155423〉.

[119] R.T. Tung, Appl. Phys. Rev. 1 (1) (2014) 011304, http://dx.doi.org/10.1063/1.4858400 URL 〈http://scitation.aip.org/con tent/aip/journal/apr2/1/1/10.1063/1.4858400〉.

[120] H. Stöcker, M. Zschornak, J. Seibt, F. Hanzig, S. Wintz, B. Abendroth,

J. Kortus, D. Meyer, Appl. Phys. A 100 (2) (2010) 437-445, http://dx.doi.org/10.1007/s00339-010-5848-0 URL 〈http:// dx.doi.org/10. 1007/s00339-010-5848-0).

[121] S.A. Chambers, M. Gu, P.V. Sushko, H. Yang, C. Wang, N. D. Browning, Adv. Mater. 25 (29) (2013) 4001-4005, http://dx.doi.org/10.1002/adma.201301030 URL 〈http:// dx.doi.org/10. 1002/adma.201301030〉.

[122] C. Capan, G.Y. Sun, M.E. Bowden, S.A. Chambers, Appl. Phys. Lett. 100 (5) (2012) 052106, http://dx.doi.org/10.1063/1.3680608 URL 〈http://scitation.aip.org/content/aip/journal/apl/100/5/10.1063/1. 3680608>

[123] C. Lenser, A. Köhl, M. Patt, C.M. Schneider, R. Waser, R. Dittmann, Phys. Rev. B 90 (2014) 115312, http://dx.doi.org/10.1103/PhysRev B.90.115312 URL 〈http://link.aps.org/doi/10.1103/PhysRevB.90. $115312\rangle$.

[124] T. Jaouen, G. Jézéquel, G. Delhaye, B. Lépine, P. Turban, P. Schieffer, Appl. Phys. Lett. 97 (23) (2010) 232104, http://dx.doi.org/10.1063/1.3525159 URL 〈http://scitation.aip.org/con tent/aip/journal/apl/97/23/10.1063/1.3525159 .

[125] T. Jaouen, G. Jézéquel, G. Delhaye, B. Lépine, P. Turban, P. Schieffer, Appl. Phys. Lett. 100 (2) (2012) 022103, http://dx.doi.org/10.1063/ 1.3675859 URL 〈http://scitation.aip.org/content/aip/journal/apl/100/2/10. $1063 / 1.3675859\rangle$.

[126] T. Jaouen, P. Aebi, S. Tricot, G. Delhaye, B. Lépine, D. Sébilleau, G. Jézéquel, P. Schieffer, Phys. Rev. B 90 (2014) 125433, http://dx.doi.org/10.1103/PhysRevB.90.125433 URL 〈http://link.aps. org/doi/10.1103/PhysRevB.90.125433〉.

[127] M. Yoshitake, S. Nemšák, T. Skála, N. Tsud, T. Kim, V. Matolín, K. C. Prince, Surf. Sci. 604 (2324) (2010) 2150-2156, http://dx.doi.org/ 10.1016/j.susc.2010.09.007 URL 〈http://www.sciencedirect.com/science/arti cle/pii/S0039602810003833〉.

[128] M. Yoshitake, W. Song, J. Libra, K. Mašek, F. Šutara, V. Matolín, K. C. Prince, J. Appl. Phys. 103 (3) (2008) 033707, http://dx.doi.org/ 10.1063/1.2837116 URL 〈http://scitation.aip.org/content/aip/journal/jap/ $103 / 3 / 10.1063 / 1.2837116\rangle$

[129] A. Morosov, A. Sigov, Phys. Solid State 54 (2) (2012) 219-242, http://dx.doi.org/10.1134/S1063783412020187 URL 〈http://dx.doi.org/ 10.1134/S1063783412020187>

[130] J. Velev, P. Dowben, E. Tsymbal, S. Jenkins, A. Caruso, Surf. Sci. Rep. 63 (9) (2008) 400-425, http://dx.doi.org/10.1016/j.surfrep.2008.06.002 URL 〈http://www.sciencedirect.com/science/article/pii/S0167572908000514〉.

[131] E. Fawcett, Rev. Mod. Phys. 60 (1988) 209-283, http:// dx.doi.org/ 10.1103/RevModPhys.60.209 URL 〈http://link.aps.org/ doi/10.1103/Rev ModPhys.60.209〉.

[132] P.J. Brown, J.B. Forsyth, E. Lelivre-Berna, F. Tasset, J. Phys.: Condens. Matter 14 (8) (2002) 1957 URL 〈http://stacks.iop.org/0953-8984/14/i=8/a=323〉.

[133] D.J. Huang, H.-T. Jeng, C.F. Chang, G.Y. Guo, J. Chen, W.P. Wu, S. C. Chung, S.G. Shyu, C.C. Wu, H.-J. Lin, C.T. Chen, Phys. Rev. B 66 (2002) 174440, http://dx.doi.org/10.1103/PhysRevB.66.174440 URL 〈http://link.aps. org/doi/10.1103/PhysRevB.66.174440〉.

[134] O. Eriksson, A.M. Boring, R.C. Albers, G.W. Fernando, B.R. Cooper, Phys. Rev. B 45 (1992) 2868-2875, http://dx.doi.org/10.1103/PhysRev B.45.2868 URL 〈http://link.aps.org/doi/10.1103/PhysRevB.45.2868〉.

[135] W.L. Roth, Phys. Rev. 110 (1958) 1333-1341, http://dx.doi.org/ 10.1103/PhysRev.110.1333 URL 〈http://link.aps.org/doi/10.1103/Phys Rev.110.1333〉.

[136] Z. Zhang, S. Satpathy, Phys. Rev. B 44 (1991) 1331913331, http://dx.doi.org/10.1103/PhysRevB.44.13319 URL 〈http:// link.aps.org/doi/ 10.1103/PhysRevB.44.13319〉.

[137] D.J. Huang, C.F. Chang, H.-T. Jeng, G.Y. Guo, H.-J. Lin, W.B. Wu, H. C. Ku, A. Fujimori, Y. Takahashi, C.T. Chen, Phys. Rev. Lett. 93 (2004) 077204, http://dx.doi.org/10.1103/PhysRevLett.93.077204 URL 〈http://link. aps.org/doi/10.1103/PhysRevLett.93.077204〉.

[138] A.K. Cheetham, D.A.O. Hope, Phys. Rev. B 27 (1983) 6964-6967, http://dx.doi.org/10.1103/PhysRevB.27.6964 URL 〈http://link.aps.org/ doi/10.1103/PhysRevB.27.6964).

[139] P. Luches, L. Pasquini, S. Benedetti, V. Bellini, S. Valeri, F. Manghi, R. Rüffer, F. Boscherini, Phys. Rev. B 83 (2011) 094413, http://dx.doi.org/10.1103/PhysRevB.83.094413 URL 〈http://link.aps. org/doi/10.1103/PhysRevB.83.094413

[140] W.H. Meiklejohn, C.P. Bean, Phys. Rev. 102 (1956) 1413-1414, http://dx.doi.org/10.1103/PhysRev.102.1413 URL 〈http://link.aps.org/ doi/10.1103/PhysRev.102.1413〉. 
[141] M. Finazzi, Phys. Rev. B 69 (2004) 064405, http://dx.doi.org/10.1103/PhysRevB.69.064405 URL 〈http://link.aps. org/doi/10.1103/PhysRevB.69.064405〉.

[142] H. Ohldag, T.J. Regan, J. Stöhr, A. Scholl, F. Nolting, J. Lüning, C. Stamm, S. Anders, R.L. White, Phys. Rev. Lett. 87 (2001) 247201, http://dx.doi.org/10.1103/PhysRevLett.87.247201 URL 〈http://link.aps. org/doi/10.1103/PhysRevLett.87.247201>.

[143] E. Mlyńczak, J. Gurgul, J. Przewoźnik, D. Wilgocka-Ślzak, K. Freindl, N. Spiridis, J. Korecki, Appl. Surf. Sci. 304 (2014) 86-90, http://dx.doi.org/10.1016/j.apsusc.2014.01.167 URL 〈http://www.scien cedirect.com/science/article/pii/S0169433214002190〉.

[144] Y. Fan, K.J. Smith, G. Luepke, A.T. Hanbicki, R. Goswami, C.H. Li, H. B. Zhao, B.T. Jonker, Nat. Nanotechnol. 8 (6) (2013) 438-444, http://dx.doi.org/10.1038/NNANO.2013.94.

[145] F. Allegretti, G. Parteder, M. Ramsey, S. Surnev, F. Netzer, Surf. Sci. 601 (14) (2007) L73-L76, http://dx.doi.org/10.1016/j.susc.2007.05.008 URL 〈http://www.sciencedirect.com/science/article/pii/S0039602807005560〉.

[146] R. de Masi, D. Reinicke, F. Müller, P. Steiner, S. Hüfner, Surf. Sci 516 (12) (2002) L515-L521, http://dx.doi.org/10.1016/S0039-6028(02) 01906-4 URL 〈http://www.sciencedirect.com/science/article/pii/ S0039602802019064).

[147] M. Bäumer, D. Cappus, H. Kuhlenbeck, H.-J. Freund, G. Wilhelmi, A. Brodde, H. Neddermeyer, Surf. Sci. 253 (13) (1991) 116-128, http://dx.doi.org/10.1016/0039-6028(91)90585-G URL 〈http://www. sciencedirect.com/science/article/pii/003960289190585G $\rangle$

[148] G. Bussetti, M. Riva, A. Picone, A. Brambilla, L. Duò, M. Finazzi, F. Ciccacci, New J. Phys. 14 (5) (2012) 053048 URL 〈http://stacks.iop.org/ $1367-2630 / 14 / i=5 / a=053048>$.

[149] A.V. Mijiritskii, P.J.M. Smulders, V.Y. Chumanov, O.C. Rogojanu, M. A. James, D.O. Boerma, Phys. Rev. B 58 (1998) 8960-8966, http://dx.doi.org/10.1103/PhysRevB.58.8960 URL 〈http://link.aps.org/ doi/10.1103/PhysRevB.58.8960).

[150] G. Bussetti, M. Riva, A. Picone, A. Brambilla, L. Duò, F. Ciccacci, M. Finazzi, Nanosci. Nanotechnol. Lett. 4 (11) (2012) 1092-1095, http://dx.doi.org/10.1166/nnl.2012.1463.

[151] S.K. Kim, C. Petersen, F. Jona, P.M. Marcus, Phys. Rev. B 54 (1996) 2184-2190, http://dx.doi.org/10.1103/PhysRevB.54.2184 URL /http:// link.aps.org/doi/10.1103/PhysRevB.54.2184〉.

[152] L. Duò, R. Bertacco, G. Isella, F. Ciccacci, M. Richter, Phys. Rev. B 61 (2000) 15294-15301, http://dx.doi.org/10.1103/PhysRevB.61.15294 URL 〈http://link. aps.org/doi/10.1103/PhysRevB.61.15294〉.

[153] A. Picone, M. Riva, G. Fratesi, A. Brambilla, G. Bussetti, M. Finazzi, L. Duò, F. Ciccacci, Phys. Rev. Lett. 113 (2014) 046102, http://dx.doi.org/10.1103/PhysRevLett.113.046102 URL 〈http://link. aps.org/doi/10.1103/PhysRevLett.113.046102 .

[154] S. Valeri, A. Borghi, G. Gazzadi, A. di Bona, Surf. Sci. 423 (23) (1999) 346-356.

[155] G. Gazzadi, A. Borghi, A. di Bona, S. Valeri, Surf. Sci. 402-404 (1998) 632-635, http://dx.doi.org/10.1016/S0039-6028(97)00920-5 URL 〈http://www.sciencedirect.com/science/article/pii/S0039602897009205〉.

[156] O. Dugerjav, H. Kim, J.M. Seo, AIP Adv. 1 (3) (2011) 032156 , http://dx.doi.org/10.1063/1.3642601 URL 〈http://scitation.aip.org/con tent/aip/journal/adva/1/3/10.1063/1.3642601 $\rangle$.

[157] J.C. Read, P.G. Mather, R.A. Buhrman, Appl. Phys. Lett. 90 (13) (2007) 132503, http://dx.doi.org/10.1063/1.2717091 URL 〈http://scitation.aip. org/content/aip/journal/apl/90/13/10.1063/1.2717091>.

[158] M. Xue, Q. Guo, J. Alloys Compd. 598 (2014) 224-229, http://dx.doi.org/10.1016/j.jallcom.2014.02.023 URL 〈http://www.scien cedirect.com/science/article/pii/S092583881400351X).

[159] M. Xue, Q. Guo, K. Wu, J. Guo, J. Cryst. Growth 311 (15) (2009) 3918-3923, http://dx.doi.org/10.1016/j.jcrysgro.2009.06.001 URL $\langle$ http://www.sciencedirect.com/science/article/pii/S0022024809005776〉.

[160] M. Kamiko, R. Yamamoto, J. Cryst. Growth 293 (1) (2006) 216-222, http://dx.doi.org/10.1016/j.jcrysgro.2006.05.009 URL 〈http:// www.sciencedirect.com/science/article/pii/S0022024806004933〉.

[161] T. Nozaki, T. Ohkubo, Y. Shiota, H. Kubota, A. Fukushima, K. Hono, Y. Suzuki, S. Yuasa, Appl. Phys. Express 6 (11) (2013) 113004 URL 〈http://stacks.iop.org/1882-0786/6/i=11/a =113004〉.
[162] T. Nozaki, H. Kubota, A. Fukushima, S. Yuasa, Appl. Phys. Lett. 106 (2) (2015) 022405, http://dx.doi.org/10.1063/1.4905927 URL 〈http://scitation.aip.org/content/aip/journal/ap1/106/2/10.1063/1.4905927〉.

[163] P. Torelli, S. Benedetti, P. Luches, L. Gragnaniello, J. Fujii, S. Valeri, Phys. Rev. B 79 (2009) 035408, http://dx.doi.org/10.1103/PhysRev B.79.035408 URL 〈http://link.aps.org/doi/10.1103/PhysRevB.79.035408〉.

[164] S. Altieri, S.F. Contri, S. Valeri, Phys. Rev. B 76 (2007) 205413, http://dx.doi.org/10.1103/PhysRevB.76.205413 URL 〈http:// link.aps.org/doi/10.1103/PhysRevB.76.205413〉.

[165] J. Jung, H.-J. Shin, Y. Kim, M. Kawai, Phys. Rev. B 82 (2010) 085413, http://dx.doi.org/10.1103/PhysRevB.82.085413 URL 〈http:// link.aps.org/doi/10.1103/PhysRevB.82.085413〉.

[166] L. Savio, E. Celasco, L. Vattuone, M. Rocca, J. Phys. Chem. B 108 (23) (2004) 7771-7778, http://dx.doi.org/10.1021/jp0360873.

[167] L. Savio, E. Celasco, L. Vattuone, M. Rocca, J. Chem. Phys. 119 (23) (2003) 12053-12056, http://dx.doi.org/10.1063/1.1633751 URL 〈http://scitation.aip. org/content/aip/journal/jcp/119/23/10.1063/1.1633751>.

[168] Y.-N. Sun, Z.-H. Qin, M. Lewandowski, E. Carrasco, M. Sterrer, S. Shaikhutdinov, H.-J. Freund, J. Catal. 266 (2) (2009) 359-368, http://dx.doi.org/10.1016/j.jcat.2009.07.002 URL 〈http://www.science direct.com/science/article/pii/S0021951709002255〉.

[169] F. Ringleb, Y. Fujimori, M.A. Brown, W.E. Kaden, F. Calaza, H. Kuhlenbeck, M. Sterrer, H.-J. Freund, Catal. Today 240, Part B (2015) 206-213, http://dx.doi.org/10.1016/j.cattod.2014.04.025, URL 〈http:// www.sciencedirect.com/science/article/pii/S0920586114003514〉.

[170] N. Nilius, Surf. Sci. Rep. 64 (12) (2009) 595-659, http://dx.doi.org/10.1016/j.surfrep.2009.07.004 URL /http://www.scien cedirect.com/science/article/pii/S0167572909000491〉.

[171] M. Sierka, Prog. Surf. Sci. 85 (912) (2010) 398-434 http://dx.doi.org/10.1016/j.progsurf.2010.07.004 URL 〈http://www. sciencedirect.com/science/article/pii/S0079681610000237〉.

[172] A. Brambilla, A. Picone, M. Finazzi, L. Duò, F. Ciccacci, Surf. Sci. 605 (12) (2011) 95-100, http://dx.doi.org/10.1016/j.susc.2010.10.005 URL 〈http://www.sciencedirect.com/science/article/pii/S0039602810004024〉.

[173] S. Ekelund, C. Leygraf, Surf. Sci. 40 (1) (1973) 179-199, http://dx.doi.org/10.1016/0039-6028(73)90061-7 URL 〈http://www. sciencedirect.com/science/article/pii/0039602873900617〉.

[174] A. Stierle, P. Bdeker, H. Zabel, Surf. Sci. 327 (12) (1995) 9-16, http://dx.doi.org/10.1016/0039-6028(94)00830-2 URL 〈http:// www.sciencedirect.com/science/article/pii/0039602894008302 $\rangle$.

[175] M. Müller, H. Oechsner, Surf. Sci. 387 (13) (1997) 269-278, http://dx.doi.org/10.1016/S0039-6028(97)00363-4 URL 〈http://www. sciencedirect.com/science/article/pii/S0039602897003634〉.

[176] A. Stierle, T. Koll, H. Zabel, Phys. Rev. B 58 (1998) 5062-5069, http://dx.doi.org/10.1103/PhysRevB.58.5062 URL 〈http://link.aps.org/ doi/10.1103/PhysRevB.58.5062〉.

[177] V. Maurice, S. Cadot, P. Marcus, Surf. Sci. 458 (13) (2000) 195-215, http://dx.doi.org/10.1016/S0039-6028(00)00439-8 URL 〈http://www. sciencedirect.com/science/article/pii/S0039602800004398>.

[178] H. Hagiwara, S. Koya, M. Wilde, M. Matsumoto, T. Okano, K. Fukutani, Surf. Sci. 600 (16) (2006) 3252-3257, http://dx.doi.org/ 10.1016/j.susc.2006.06.013 URL 〈http://www.sciencedirect.com/ science/article/pii/S0039602806007023〉.

[179] P. Michel, C. Jardin, Surf. Sci. 36 (2) (1973) 478-487, http://dx.doi.org/10.1016/0039-6028(73)90396-8 URL 〈http://www. sciencedirect.com/science/article/pii/0039602873903968〉.

[180] M. Bridge, R. Lambert, Surf. Sci. 82 (2) (1979) 413-424, http://dx.doi.org/10.1016/0039-6028(79)90199-7 URL 〈http://www. sciencedirect.com/science/article/pii/0039602879901997〉.

[181] S. Nemšák, T. Skála, M. Yoshitake, K.C. Prince, V. Matolín, J. Phys.: Condens. Matter 25 (9) (2013) 095004 URL /http://stacks.iop.org/ $0953-8984 / 25 / \mathrm{i}=9 / \mathrm{a}=095004\rangle$.

[182] X. Chen, H. Kazi, Y. Cao, B. Dong, F.L. Pasquale, J.A.C. Santana, S. Cao, M. Street, R. Welch, C. Binek, A. Enders, J.A. Kelber, P. Dowben, Mater. Chem. Phys. 149-150 (2015) 113-123, http://dx.doi.org/10.1016/j.matchemphys.2014.09.053 URL 〈http://www. sciencedirect.com/science/article/pii/S025405841400635X). 
[183] Y.-Z. Wang, M. Yang, D.-C. QI, S. Chen, W. Chen, A.T.S. Wee, X.Y. Gao, J. Chem. Phys. 134 (3) (2011) 034706 10.1063/1.3546034 URL 〈http://scitation.aip.org/content/aip/journal/jcp/134/3/10.1063/1.3546034〉.

[184] J.I. Flege, A. Meyer, J. Falta, E.E. Krasovskii, Phys. Rev. B 84 (2011) 115441, http://dx.doi.org/10.1103/PhysRevB.84.115441 URL 〈http:// link.aps.org/doi/10.1103/PhysRevB.84.115441〉.

[185] D.F. Forster, J. Klinkhammer, C. Busse, S.G. Altendorf, T. Michely, Z. Hu, Y.-Y. Chin, L.H. Tjeng, J. Coraux, D. Bourgault, Phys. Rev. B 83 (2011) 045424, http://dx.doi.org/10.1103/PhysRevB.83.045424 URL 〈http://link.aps.org/doi/10.1103/PhysRevB.83.045424〉.

[186] D.F. Förster, J. Klinkhammer, T. Michely, Surf. Sci. 606 (1314) (2012) 1019-1028, http://dx.doi.org/10.1016/j.susc.2012.02.021 URL 〈http:// www.sciencedirect.com/science/article/pii/S0039602812000726〉.

[187] S.L. Pévédic, D. Schmaus, C. Cohen, Surf. Sci. 602 (1) (2008) 67-76, http://dx.doi.org/10.1016/j.susc.2007.09.050 URL 〈http://www. sciencedirect.com/science/article/pii/S0039602807009752〉.

[188] S. Nemsak, T. Skala, M. Yoshitake, N. Tsud, T. Kim, S. Yagyu, V. Matolin, Surf. Interface Anal. 42 (10-11) (2010) 1581-1584 http://dx.doi.org/10.1002/sia.3582.

[189] G. Prévot, S. Le Moal, R. Bernard, B. Croset, R. Lazzari, D. Schmaus, Phys. Rev. B 85 (2012) 205450, http://dx.doi.org/10.1103/ PhysRevB.85.205450 URL 〈http://link.aps.org/doi/10.1103/PhysRevB. $85.205450\rangle$.

[190] A.C. Papageorgiou, G. Cabailh, Q. Chen, A. Resta, E. Lundgren, J. N. Andersen, G. Thornton, J. Phys. Chem. C 111 (21) (2007) 7704-7710, http://dx.doi.org/10.1021/jp067802m.

[191] K. Kishi, K. Fujiwara, J. Electron Spectrosc. Relat. Phenom. 85 (12) (1997) 123-134, http://dx.doi.org/10.1016/S0368-2048(96)03093-9 URL 〈http:// www.sciencedirect.com/science/article/pii/S0368204896030939〉.

[192] R.I.R. Blyth, C. Searle, N.P. Tucker, S.D. Barrett, Phys. Rev. B 70 (2004) 045402, http://dx.doi.org/10.1103/PhysRevB.70.045402 URL /http://link.aps. org/doi/10.1103/PhysRevB.70.045402

[193] C. Gu, C.G. Olson, D.W. Lynch, Phys. Rev. B 48 (1993) 12178-12182, http://dx.doi.org/10.1103/PhysRevB.48.12178 URL /http://link.aps.org/ doi/10.1103/PhysRevB.48.12178>.

[194] D. Riffe, G. Wertheim, Surf. Sci. 399 (23) (1998) 248-263 http://dx.doi.org/10.1016/S0039-6028(97)00824-8 URL 〈http://www. sciencedirect.com/science/article/pii/S0039602897008248>.

[195] P. Nordlander, M. Ronay, Phys. Rev. B 36 (1987) 4982-4989, http://dx.doi.org/10.1103/PhysRevB.36.4982 URL 〈http://link.aps.org/ doi/10.1103/PhysRevB.36.4982 .

[196] M. Ronay, P. Nordlander, Phys. Rev. B 35 (1987) 9403-9406 http://dx.doi.org/10.1103/PhysRevB.35.9403 URL 〈http://link.aps.org/ doi/10.1103/PhysRevB.35.9403 .

[197] K. Radican, S. Bozhko, S.-R. Vadapoo, S. Ulucan, H.-C. Wu, A. McCoy, I. Shvets, Surf. Sci. 604 (1920) (2010) 1548-1551, http://dx.doi.org/10.1016/j.susc.2010.05.016 URL〈http://www.science direct.com/science/article/pii/S0039602810002189〉.

[198] K. Radican, N. Berdunov, G. Manai, I.V. Shvets, Phys. Rev. B 75 (2007) 155434, http://dx.doi.org/10.1103/PhysRevB.75.155434 URL /http://link.aps. org/doi/10.1103/PhysRevB.75.155434>.

[199] M. Gallagher, M. Fyfield, J. Cowin, S. Joyce, Surf. Sci. 339 (3) (1995) L909-L913, http://dx.doi.org/10.1016/0039-6028(95)80056-5 URL 〈http://www.sciencedirect.com/science/article/pii/0039602895800565〉.

[200] M. Gallagher, M. Fyfield, L. Bumm, J. Cowin, S. Joyce, Thin Solid Films 445 (1) (2003) 90-95, http://dx.doi.org/10.1016/j.tsf.2003.09.016 URL 〈http://www.sciencedirect.com/science/article/pii/S0040609003012872〉.

[201] H. Benia, P. Myrach, N. Nilius, H.-J. Freund, Surf. Sci. 604 (34) (2010) 435-441, http://dx.doi.org/10.1016/j.susc.2009.12.011 URL 〈http:// www.sciencedirect.com/science/article/pii/S0039602809007754〉.

[202] Q. Guo, W. Oh, D. Goodman, Surf. Sci. 437 (12) (1999) 49-60, http://dx.doi.org/10.1016/S0039-6028(99)00678-0 URL 〈http:// www.sciencedirect.com/science/article/pii/S0039602899006780〉.

[203] C.L. Pang, D.C. Grinter, J. Matharu, G. Thornton, J. Phys. Chem. C 117 (48) (2013) 25622-25627, http://dx.doi.org/10.1021/jp409948u.

[204] J. Matharu, G. Cabailh, G. Thornton, Surf. Sci. 616 (2013) 198-205, http://dx.doi.org/10.1016/j.susc.2013.05.020 URL 〈http:// www.sciencedirect.com/science/article/pii/S0039602813001726〉.
[205] Y. Law, T. Dintzer, S. Zafeiratos, Appl. Surf. Sci. 258 (4) (2011) 1480-1487, http://dx.doi.org/10.1016/j.apsusc.2011.09.111 URL 〈http:// www.sciencedirect.com/science/article/pii/S0169433211015145〉.

[206] A.M. Silva, C. Achete, R.B. Capaz, Chem. Phys. 410 (2013) 99-102, http://dx.doi.org/10.1016/j.chemphys.2012.11.004 URL 〈http://www. sciencedirect.com/science/article/pii/S0301010412004326).

[207] S. Zafeiratos, S. Piccinin, D. Teschner, Catal. Sci. Technol. 2 (2012) 1787-1801, http://dx.doi.org/10.1039/C2CY00487A.

[208] M. Antlanger, W. Mayr-Schmölzer, J.C.V. Pavelec, F. Mittendorfer, J. Redinger, P. Varga, U. Diebold, M. Schmid, Phys. Rev. B 86 (2012) 035451, http://dx.doi.org/10.1103/PhysRevB.86.035451 URL 〈http:// link.aps.org/doi/10.1103/PhysRevB.86.035451 .

[209] J.I.J. Choi, W. Mayr-Schmölzer, F. Mittendorfer, J. Redinger, U. Diebold, M. Schmid, J. Phys.: Condens. Matter 26 (22) (2014) 225003 URL $\langle$ http://stacks.iop.org/0953-8984/26/i $=22 / \mathrm{a}=225003\rangle$. D

[210] Flötotto, Z. Wang, E. Mittemeijer, Surf. Sci. 633 (2015) 1-7, http://dx.doi.org/10.1016/j.susc.2014.11.008 URL 〈http://www.science direct.com/science/article/pii/S0039602814003161〉.

[211] J. Crowell, J. Chen, J.Y. Jr., Surf. Sci. 165(1)(1986) 37-64, http://dx. doi.org/10.1016/0039-6028(86)90663-1,URL 〈http://www.sciencedirect. com/science/article/pii/0039602886906631 .

[212] R. Jaeger, H. Kuhlenbeck, H.-J. Freund, M. Wuttig, W. Hoffmann, R. Franchy, H. Ibach, Surf. Sci. 259 (3) (1991) 235-252, http://dx.doi.org/10.1016/0039-6028(91)90555-7 URL 〈http://www. sciencedirect.com/science/article/pii/0039602891905557〉.

[213] G. Kresse, M. Schmid, E. Napetschnig, M. Shishkin, L. Köhler, P. Varga, Science 308 (5727) (2005) 1440-1442, http://dx.doi.org/ 10.1126/science.1107783 URL 〈http://www.sciencemag.org/content/ 308/5727/1440.abstract $\rangle$.

[214] A. Rosenhahn, J. Schneider, J. Kandler, C. Becker, K. Wandelt, Surf. Sci. 433-435 (1999) 705-710, http://dx.doi.org/10.1016/ S0039-6028(99)00126-0 URL 〈http://www.sciencedirect.com/science/ article/pii/S0039602899001260〉.

[215] M. Yoshitake, S. Bera, Y. Yamauchi, Surf. Interface Anal. 35 (10) (2003) 824-828, http://dx.doi.org/10.1002/sia.1610.

[216] D.V. Potapenko, R.M. Osgood, Nano Letters 9 (6) (2009) 2378-2383, http://dx.doi.org/10.1021/n1900904s.

[217] M. De Santis, A. Buchsbaum, P. Varga, M. Schmid, Phys. Rev. B 84 (2011) 125430, http://dx.doi.org/10.1103/PhysRevB.84.125430 URL 〈http://link.aps. org/doi/10.1103/PhysRevB.84.125430〉.

[218] J.A. Stroscio, D.T. Pierce, R.A. Dragoset, Phys. Rev. Lett. 70 (1993) 3615-3618, http://dx.doi.org/10.1103/PhysRevLett.70.3615 URL $\langle$ http://link.aps.org/doi/10.1103/PhysRevLett.70.3615〉.

[219] D. Pierce, J. Unguris, R. Celotta, M. Stiles, J. Magn. Magn. Mater. 200 (13) (1999) 290-321, http://dx.doi.org/10.1016/S0304-8853(99)00319-4 URL 〈http://www.sciencedirect.com/science/article/pii/S0304885399003194〉.

[220] M. Klaua, D. Ullmann, J. Barthel, W. Wulfhekel, J. Kirschner, R. Urban, T.L. Monchesky, A. Enders, J.F. Cochran, B. Heinrich, Phys. Rev. B 64 (2001) 134411, http://dx.doi.org/10.1103/PhysRevB.64. 134411 URL 〈http://link.aps.org/doi/10.1103/PhysRevB.64.134411〉.

[221] R. Bertacco, S. De Rossi, F. Ciccacci, J. Vac. Sci. Technol. A 16 (4), (1998).

[222] F. Bonell, A.M. Bataille, S. Andrieu, C. Tiusan, B. Kierren, G. Lengaigne, D. Lacour, Eur. Phys. J. Appl. Phys. 43 (3) (2008) 357-361, http://dx.doi.org/10.1051/epjap:2008100.

[223] A. Picone, A. Brambilla, A. Calloni, L. Duò, M. Finazzi, F. Ciccacci, Phys. Rev. B 83 (2011) 235402, http://dx.doi.org/10.1103/ PhysRevB.83.235402 URL〈http://link.aps.org/doi/10.1103/PhysRevB. 83.235402).

[224] J.-P. Lu, M.R. Albert, S.L. Bernasek, D.J. Dwyer, Surf. Sci. 215 (3) (1989) 348-362, http://dx.doi.org/10.1016/0039-6028(89)90265-3 URL 〈http://www. sciencedirect.com/science/article/pii/0039602889902653〉.

[225] A. Tange, C.L. Gao, B.Y. Yavorsky, I.V. Maznichenko, C. Etz, A. Ernst, W. Hergert, I. Mertig, W. Wulfhekel, J. Kirschner, Phys. Rev. B 81 (2010) 195410, http://dx.doi.org/10.1103/PhysRevB.81.195410 URL 〈http://link.aps.org/doi/10.1103/PhysRevB.81.195410〉.

[226] F. Donati, P. Sessi, S. Achilli, A. Li Bassi, M. Passoni, C.S. Casari, C. E. Bottani, A. Brambilla, A. Picone, M. Finazzi, L. Duò, M.I. Trioni, 
F. Ciccacci, Phys. Rev. B 79 (2009) 195430, http://dx.doi.org/10.1103/ PhysRevB.79.195430 URL 〈http://link.aps.org/doi/10.1103/PhysRevB. $79.195430\rangle$.

[227] A. Picone, G. Fratesi, A. Brambilla, P. Sessi, F. Donati, S. Achilli, L. Maini, M.I. Trioni, C.S. Casari, M. Passoni, A. Li Bassi, M. Finazzi, L. Duò, F. Ciccacci, Phys. Rev. B 81 (2010) 115450, http://dx.doi.org/10.1103/PhysRevB.81.115450 URL 〈http://link.aps. org/doi/10.1103/PhysRevB.81.115450).

[228] W. Hofer, J. Redinger, A. Biedermann, P. Varga, Surf. Sci 466 (13) (2000) L795-L801, http://dx.doi.org/10.1016/S0039-6028(00)00821-9 URL 〈http://www.sciencedirect.com/science/article/pii/S0039602800008219).

[229] J.A. Stroscio, D.T. Pierce, J. Vac. Sci. Technol. B 12 (3), (1994).

[230] H.W. Hugosson, W. Cao, S. Seetharaman, A. Delin, J. Phys. Chem. C 117 (12) (2013) 6161-6171, http://dx.doi.org/10.1021/jp3102496.

[231] P. Błoński, A. Kiejna, J. Hafner, Surf. Sci. 590 (1) (2005) 88-100, http:// dx.doi.org/10.1016/j.susc.2005.06.011 URL $\langle$ http://www.science direct.com/science/article/pii/S0039602805006539〉.

[232] S.R. Chubb, W.E. Pickett, Phys. Rev. Lett. 58 (1987) 12481251, http://dx.doi.org/10.1103/PhysRevLett.58.1248 URL /http://link. aps.org/doi/10.1103/PhysRevLett.58.1248>.

[233] K.O. Legg, F. Jona, D.W. Jepsen, P.M. Marcus, Phys. Rev. B 16 (1977) 5271-5276, http://dx.doi.org/10.1103/PhysRevB.16.5271 URL 〈http:// link.aps.org/doi/10.1103/PhysRevB.16.5271>.

[234] S.S. Parihar, H.L. Meyerheim, K. Mohseni, S. Ostanin, A. Ernst, N. Jedrecy, R. Felici, J. Kirschner, Phys. Rev. B 81 (2010) 075428, http://dx.doi.org/10.1103/PhysRevB.81.075428 URL 〈http://link.aps. org/doi/10.1103/PhysRevB.81.075428>.

[235] R. Bertacco, F. Ciccacci, Phys. Rev. B 59 (1999) 4207-4210, http://dx.doi.org/10.1103/PhysRevB.59.4207 URL 〈http:// link.aps.org/doi/10.1103/PhysRevB.59.4207).

[236] F. Bisio, R. Moroni, M. Canepa, L. Mattera, R. Bertacco, F. Ciccacci, Phys. Rev. Lett. 83 (1999) 4868-4871, http://dx.doi.org/10.1103/ PhysRevLett.83.4868 URL 〈http://link.aps.org/doi/10.1103/PhysRev Lett.83.4868>.

[237] M. Nývlt, F. Bisio, J. Franta, C.L. Gao, H. Petek, J. Kirschner, Phys. Rev. Lett. 95 (2005) 127201, http://dx.doi.org/10.1103/ PhysRevLett.95.127201 URL 〈http://link.aps.org/doi/10.1103/ PhysRevLett.95.127201>.

[238] W. Feng, H.L. Meyerheim, K. Mohseni, O. Brovko, V.S. Stepanyuk, N. Jedrecy, R. Felici, J. Kirschner, Phys. Rev. Lett. 110 (2013) 235503, http://dx.doi.org/10.1103/PhysRevLett.110.235503 URL 〈http://link.aps.org/doi/10.1103/PhysRevLett.110.235503〉.

[239] A.G. Sault, Appl. Surf. Sci. 74 (3) (1994) 249-262, http://dx.doi.org/10.1016/0169-4332(94)90006-X URL 〈http://www. sciencedirect.com/science/article/pii/016943329490006X $\rangle$.

[240] M. Seo, J. Lumsden, R. Staehle, Surf. Sci. 50 (2) (1975) 541-552, http://dx.doi.org/10.1016/0039-6028(75)90043-6 URL 〈http://www. sciencedirect.com/science/article/pii/0039602875900436〉.

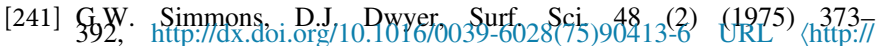
www.sciencedirect.com/science/article/pii/0039602875904136).

[242] K. Freindl, E. Partyka-Jankowska, W. Karaś, M. Zając, E. Madej, N. Spiridis, M. Ślęzak, T. Ślęzak, D. Wiśnios, J. Korecki, Surf. Sci. 617 (2013) 183-191, http://dx.doi.org/10.1016/j.susc.2013.07.011 URL 〈http://www.sciencedirect. com/science/article/pii/S0039602813002021〉.

[243] J.A. Stroscio, D.T. Pierce, A. Davies, R.J. Celotta, M. Weinert, Phys. Rev. Lett. 75 (1995) 2960-2963, http://dx.doi.org/10.1103/ PhysRevLett.75.2960 URL 〈http://link.aps.org/doi/10.1103/PhysRev Lett.75.2960).

[244] M.M.J. Bischoff, T.K. Yamada, C.M. Fang, R.A. de Groot, H. van Kempen, Phys. Rev. B 68 (2003) 045422, http://dx.doi.org/10.1103/ PhysRevB.68.045422 URL 〈http://link.aps.org/doi/10.1103/PhysRevB. $68.045422\rangle$.

[245] V.A. Ukraintsev, Phys. Rev. B 53 (1996) 11176-11185 http://dx.doi.org/10.1103/PhysRevB.53.11176 URL 〈http://link.aps.org/ doi/10.1103/PhysRevB.53.11176 .

[246] M. Passoni, F. Donati, A. Li Bassi, C.S. Casari, C.E. Bottani, Phys. Rev. B 79 (2009) 045404, http://dx.doi.org/10.1103/PhysRevB.79.045404 URL 〈http://link.aps.org/doi/10.1103/PhysRevB.79.045404〉.
[247] J. Middeke, R.-P. Blum, M. Hafemeister, H. Niehus, Surf. Sci. 587 (3) (2005) 219-228, http://dx.doi.org/10.1016/j.susc.2005.03.065 URL 〈http://www. sciencedirect.com/science/article/pii/S0039602805005236>.

[248] D. Vlachos, S.D. Foulias, M. Kamaratos, J. Phys.: Condens. Matter 21 (44) (2009) 445004 URL $\langle$ http://stacks.iop.org/0953-8984/21/i = 44/a = 445004〉.

[249] K. Amemiya, M. Sakamaki, Appl. Phys. Lett. 98 (1) (2011) 012501, http://dx.doi.org/10.1063/1.3531668 URL 〈http://scitation.aip. org/content/aip/journal/ap1/98/1/10.1063/1.3531668>.

[250] C. Wu, M.R. Castell, J. Goniakowski, C. Noguera, Phys. Rev. B 91 (2015) 155424, http://dx.doi.org/10.1103/PhysRevB.91.155424 URL 〈http://link.aps. org/doi/10.1103/PhysRevB.91.155424).

[251] A. Picone, G. Fratesi, M. Riva, G. Bussetti, A. Calloni, A. Brambilla, M. I. Trioni, L. Duò, F. Ciccacci, M. Finazzi, Phys. Rev. B 87 (2013) 085403, http://dx.doi.org/10.1103/PhysRevB.87.085403 URL 〈http:// link.aps.org/doi/10.1103/PhysRevB.87.085403〉.

[252] A. Brambilla, G. Berti, A. Calloni, A. Picone, M. Riva, G. Bussetti, S. Nappini, E. Magnano, M. Finazzi, L. Duò, F. Ciccacci, J. Appl. Phys. 114 (12), (2013) 123905 http://dx.doi.org/10.1063/1.4822164, URL 〈http://scitation.aip.org/content/aip/journal/jap/114/12/10.1063/1.4822164〉.

[253] A. Davies, J.A. Stroscio, D.T. Pierce, R.J. Celotta, Phys. Rev. Lett. 76 (1996) 4175-4178, http://dx.doi.org/10.1103/PhysRevLett.76.4175 URL /http://link. aps.org/doi/10.1103/PhysRevLett.76.4175〉.

[254] B. Nonas, K. Wildberger, R. Zeller, P.H. Dederichs, Phys. Rev. Lett. 80 (1998) 4574-4577, http://dx.doi.org/10.1103/PhysRevLett.80.4574 URL 〈http://link. aps.org/doi/10.1103/PhysRevLett.80.4574〉.

[255] J.P. Perdew, K. Burke, M. Ernzerhof, Phys. Rev. Lett. 77 (1996) 3865-3868, http://dx.doi.org/10.1103/PhysRevLett.77.3865 URL 〈http://link.aps.org/doi/10.1103/PhysRevLett.77.3865〉.

[256] J. Goniakowski, C. Noguera, Phys. Rev. B 79 (2009) 155433 http://dx.doi.org/10.1103/PhysRevB.79.155433 URL 〈http://link.aps. org/doi/10.1103/PhysRevB.79.155433 .

[257] A. Picone, G. Bussetti, M. Riva, A. Calloni, A. Brambilla, L. Duò, F. Ciccacci, M. Finazzi, Phys. Rev. B 86 (2012) 075465 http://dx.doi.org/10.1103/PhysRevB.86.075465 URL 〈http://link.aps. org/doi/10.1103/PhysRevB.86.075465 .

[258] I.O. Thomas, A. Fortunelli, Eur. Phys. J. B 75 (1) (2010) 5-13, http://dx.doi.org/10.1140/epjb/e2010-00018-5 URL 〈http://dx.doi.org/ 10.1140/epjb/e2010-00018-5>.

[259] M. Ondráček, F. Máca, J. Kudrnovský, J. Redinger, A. Biedermann, C. Fritscher, M. Schmid, P. Varga, Phys. Rev. B 74 (2006) 235437, http://dx.doi.org/10.1103/PhysRevB.74.235437 URL 〈http://link.aps. org/doi/10.1103/PhysRevB.74.235437〉.

[260] J.A. Venables, G.D.T. Spiller, M. Hanbucken, Rep. Prog. Phys. 47 (4)(1984) 399 URL $\langle$ http://stacks.iop.org/0034-4885/47/i=4/a=002〉.

[261] T. Michely, J. Krug, Springer, 2004.

[262] H. Brune, Surf. Sci. Rep. 31 (46) (1998) 125-229, http://dx.doi.org/10.1016/S0167-5729(99)80001-6 URL 〈http://www. sciencedirect.com/science/article/pii/S0167572999800016 $\rangle$.

[263] J. Evans, P. Thiel, M. Bartelt, Surf. Sci. Rep. 61 (12) (2006) 1-128, http://dx.doi.org/10.1016/j.surfrep.2005.08.004 URL 〈http://www.scien cedirect.com/science/article/pii/S0167572906000021〉.

[264] M. Copel, M.C. Reuter, E. Kaxiras, R.M. Tromp, Phys. Rev. Lett. 63 (1989) 632-635, http://dx.doi.org/10.1103/PhysRevLett.63.632 URL /http://link.aps. org/doi/10.1103/PhysRevLett.63.632〉.

[265] J. Camarero, J. Ferrón, V. Cros, L. Gómez, A.L. Vázquez de Parga, J. M. Gallego, J.E. Prieto, J.J. de Miguel, R. Miranda, Phys. Rev. Lett. 81 (1998) 850-853, http://dx.doi.org/10.1103/PhysRevLett.81.850 URL 〈http://link.aps. org/doi/10.1103/PhysRevLett.81.850〉.

[266] J. Ferrón, L. Gómez, J. Gallego, J. Camarero, J. Prieto, V. Cros, A.V. de Parga, J. de Miguel, R. Miranda, Surf. Sci. 459 (12) (2000) 135-148, http://dx.doi.org/10.1016/S0039-6028(00)00459-3 URL 〈http://www. sciencedirect.com/science/article/pii/S0039602800004593〉.

[267] M.C.G. Passeggi, J.E. Prieto, R. Miranda, J.M. Gallego, Phys. Rev. B 65 (2001) 035409, http://dx.doi.org/10.1103/PhysRevB.65.035409 URL 〈http://link.aps.org/doi/10.1103/PhysRevB.65.035409〉.

[268] J.A. Stroscio, D.T. Pierce, M.D. Stiles, A. Zangwill, L.M. Sander, Phys. Rev. Lett. 75 (1995) 4246-4249, http://dx.doi.org/10.1103/ 
PhysRevLett.75.4246 URL 〈http://link.aps.org/doi/10.1103/PhysRev Lett.75.4246>.

[269] P. Bonanno, M. Canepa, P. Cantini, R. Moroni, L. Mattera, S. Terreni, Surf. Sci. 454-456 (2000) 697-701, http://dx.doi.org/10.1016/ S0039-6028(00)00114-X URL 〈http://www.sciencedirect.com/science/ article/pii/S003960280000114X $\rangle$.

[270] M. Kamiko, H. Mizuno, H. Chihaya, J. Xu, I. Kojima, R. Yamamoto, Thin Solid Films 515 (18) (2007) 7203-7208, http://dx.doi.org/10.1016/ j.tsf.2007.02.105 URL 〈http://www.sciencedirect.com/science/article/pii/ S0040609007002799>.

[271] J. Vrijmoeth, H.A. van der Vegt, J.A. Meyer, E. Vlieg, R.J. Behm, Phys. Rev. Lett. 72 (1994) 3843-3846, http://dx.doi.org/ 10.1103/PhysRevLett.72.3843 URL 〈http://link.aps.org/doi/10.1103/ PhysRevLett.72.3843>.

[272] S. Esch, M. Hohage, T. Michely, G. Comsa, Phys. Rev. Lett. 72 (1994) 518-521, http://dx.doi.org/10.1103/PhysRevLett.72.518 URL 〈http:// link.aps.org/doi/10.1103/PhysRevLett.72.518>.

[273] M. Riva, A. Picone, D. Giannotti, A. Brambilla, G. Fratesi, G. Bussetti, L. Duò, F. Ciccacci, M. Finazzi, Phys. Rev. B 92 (2015) 115434, http://dx.doi.org/10.1103/PhysRevB.92.115434 URL 〈http://link.aps. org/doi/10.1103/PhysRevB.92.115434〉.

[274] A. Tange, C. Gao, W. Wulfhekel, J. Kirschner, Phys. Rev. B 81 (2010) 220404, http://dx.doi.org/10.1103/PhysRevB.81.220404 URL 〈http:// link.aps.org/doi/10.1103/PhysRevB.81.220404).

[275] L. Li, A. Kida, M. Ohnishi, M. Matsui, Surf. Sci. 493 (13) (2001) 120-125, http://dx.doi.org/10.1016/S0039-6028(01)01198-0 URL 〈http://www.sciencedirect.com/science/article/pii/S0039602801011980〉.

[276] R. Nünthel, T. Gleitsmann, P. Poulopoulos, A. Scherz, J. Lindner, E. Kosubek, C. Litwinski, Z. Li, H. Wende, K. Baberschke, S. Stolbov, T. Rahman, Surf. Sci. 531 (1) (2003) 53-67, http://dx.doi.org/ 10.1016/S0039-6028(03)00438-2 URL 〈http://www.sciencedirect.com/ science/article/pii/S0039602803004382).

[277] H.L. Meyerheim, D. Sander, R. Popescu, W. Pan, I. Popa, J. Kirschner, Phys. Rev. Lett. 99 (2007) 116101, http://dx.doi.org/ 10.1103/PhysRevLett.99.116101 URL 〈http://link.aps.org/doi/10.1103/ PhysRevLett.99.116101>.

[278] A. Calloni, A. Picone, A. Brambilla, M. Finazzi, L. Duò, F. Ciccacci, Surf. Sci. 605 (2324) (2011) 2092-2096, http:// dx.doi.org/10.1016/j.susc.2011.08.013 URL 〈http:// www.sciencedirect.com/science/article/pii/S0039602811003499〉.

[279] M. Riva, A. Picone, G. Bussetti, A. Brambilla, A. Calloni, G. Berti, L. Duò, F. Ciccacci, M. Finazzi, Surf. Sci. 621 (2014) 55-63, http://dx.doi.org/10.1016/j.susc.2013.10.016 URL 〈http://www.science direct.com/science/article/pii/S0039602813003129〉.
[280] A. Calloni, G. Berti, A. Brambilla, M. Riva, A. Picone, G. Bussetti, M. Finazzi, F. Ciccacci, L. Duò, J. Phys.: Condens. Matter 26 (44) (2014) 445001 URL 〈http://stacks.iop.org/0953-8984/26/i=44/a=445001〉.

[281] Y. Kim, C. Westphal, R. Ynzunza, Z. Wang, H. Galloway, M. Salmeron, M.V. Hove, C. Fadley, Surf. Sci. 416 (12) (1998) 68-111, http://dx.doi.org/10.1016/S0039-6028(98)00506-8 URL 〈http://www. sciencedirect.com/science/article/pii/S0039602898005068〉.

[282] M. Finazzi, L. Duò, F. Ciccacci (Eds.), Wiley-VCH Verlag GmbH \& Co. KGaA, Weinheim, 2010. http://dx.doi.org/10.1002/9783527630370.

[283] S. Masuda, Y. Harada, H. Kato, K. Yagi, T. Komeda, T. Miyano, M. Onchi, Y. Sakisaka, Phys. Rev. B 37 (1988) 8088-8095, http://dx.doi.org/10.1103/PhysRevB.37.8088 URL 〈http://link.aps.org/ doi/10.1103/PhysRevB.37.8088>.

[284] Y. Joseph, W. Ranke, W. Weiss, J. Phys. Chem. B 104 (14) (2000) 3224-3236, http://dx.doi.org/10.1021/jp9932012.

[285] A. Rota, S. Altieri, S. Valeri, Phys. Rev. B 79 (2009) 161401 , http://dx.doi.org/10.1103/PhysRevB.79.161401 URL 〈http://link.aps. org/doi/10.1103/PhysRevB.79.161401).

[286] M. Busch, M. Gruyters, H. Winter, Surf. Sci. 600 (13) (2006) 2778-2784, http://dx.doi.org/10.1016/j.susc.2006.05.003 URL 〈http:// www.sciencedirect.com/science/article/pii/S0039602806005991〉.

[287] M. Soldemo, Y. Niu, A. Zakharov, E. Lundgren, J. Weissenrieder, Surf. Sci. 639 (2015) 13-19, http://dx.doi.org/10.1016/j.susc.2015.04.008 URL $\langle$ http://www.sciencedirect.com/science/article/pii/S00 39602815000990>.

[288] D. Cappus, M. Haßel, E. Neuhaus, M. Heber, F. Rohr, H.-J. Freund, Surf. Sci. 337 (3) (1995) 268-277, http://dx.doi.org/10.1016/0039-6028 (95)00624-9 URL 〈http://www.sciencedirect.com/science/article/pii/ $0039602895006249\rangle$.

[289] H. Nishimura, T. Tashiro, T. Fujitani, J. Nakamura, J. Vac. Sci. Technol. A 18 (4), (2000).

[290] W. Meyer, D. Hock, K. Biedermann, M. Gubo, S. Müller, L. Hammer,

K. Heinz, Phys. Rev. Lett. 101 (2008) 016103, http://dx.doi.org/ 10.1103/PhysRevLett.101.016103 URL 〈http://link.aps.org/doi/10.1103/ PhysRevLett.101.016103〉.

[291] W. Ranke, M. Ritter, W. Weiss, Phys. Rev. B 60 (1999) 1527-1530, http://dx.doi.org/10.1103/PhysRevB.60.1527 URL 〈http:// link.aps.org/doi/10.1103/PhysRevB.60.1527〉.

[292] H. Zeuthen, W. Kudernatsch, G. Peng, L.R. Merte, L.K. Ono, L. Lammich, Y. Bai, L.C. Grabow, M. Mavrikakis, S. Wendt, F. Besenbacher, J. Phys. Chem. C 117 (29) (2013) 15155-15163, http://dx.doi.org/10.1021/jp4042638.

[293] C. Noguera, J. Phys.: Condens. Matter 12 (31) (2000) R367 URL 〈http://stacks.iop.org/0953-8984/12/i=31/a $=201\rangle$.

[294] J. Goniakowski, F. Finocchi, C. Noguera, Rep. Prog. Phys. 71 (1) (2008) 016501 URL 〈http://stacks.iop.org/0034-4885/71/i=1/a=016501〉. 